\title{
Naphthyridine-based helical foldamers and macrocycles: synthesis, cation-binding and supramolecular assemblies
}

\author{
Anne Petitjean, Louis A. Cuccia, Marc Schmutz and Jean-Marie Lehn*
}

\section{Supporting information}

Table of content:

p 2-10: Synthetic protocols, characterization of intermediates and products and references p 11-73: NMR spectra

p 74: Representative ROESY spectrum (500 MHz) for $\mathbf{1}_{\text {tertBu }}$

\begin{tabular}{|c|c|c|}
\hline Benzamidine hydrochlorides & $p 2$ & p 11-13 \\
\hline 4,6-dihydroxypyrimidines & $p 3$ & p 14-19 \\
\hline 4,6-dichloropyrimidines & p 3-4 & p 20-26 \\
\hline chloroethoxyvinylpyrimidines & $p 4-5$ & p 27 \\
\hline chloropyrimidinyl ethyl ketones & p 5 & p 28-33 \\
\hline ethoxyvinylpyrimidinyl ethyl ketones & p 6 & p $34-40$ \\
\hline acetyl pyrimidinyl ethyl ketones & p 6-7 & p 41-44 \\
\hline $\mathbf{3}_{\mathrm{nBu}}$ & & p 45-46 \\
\hline $\mathbf{3}_{\mathrm{tertBu}}, \mathbf{3}_{\mathrm{OBz}}, \mathbf{3}_{\mathrm{OH}}$ & $p 7$ & $p 47-50$ \\
\hline $4_{n B u}$ & & p 51-52 \\
\hline $\mathbf{4}_{\text {tertBu, }} \mathbf{4}_{\mathrm{OBz}}$ & $p 8$ & p 53-55 \\
\hline $5_{(\mathrm{nBu})(\mathrm{nBu})}, \mathbf{5}_{(\mathrm{nBu})(\mathrm{OBz})}$ & & p 56-57 \\
\hline $6^{\prime}{ }_{n B u}, 6_{n B u}, 6_{O C 12}$ & $p 8$ & p 58-60 \\
\hline $\mathbf{1}_{\text {tertBu, }} \mathbf{1}_{\mathrm{nBu}}$ & $p 8$ & See article text \\
\hline $\mathrm{C} 3 \mathrm{~N} 2, \mathrm{C} 22 \mathrm{~N} 3, \mathrm{C33N} 3, \mathrm{C} 343 \mathrm{~N} 3$ & p9 & p 61-66 \\
\hline ProAC3N2, ProAC33N3 & p9 & p 67-70 \\
\hline ProC3N2, ProC33N3 & p 10 & p 71-73 \\
\hline
\end{tabular}


All reagents were used as received. Dry solvents (dichloromethane, toluene, THF) were distilled on drying agents (calcium hydride, $\mathrm{Na}$, $\mathrm{Na}$ benzophenone, respectively) under argon. 4Amino-pyrimidine-5-carboxaldehyde, ${ }^{1}$ 2-aminonicotinaldehyde, ${ }^{2}$ (1-ethoxyvinyl)-tris( $n$-butyl)stannane, ${ }^{3} 4$ - $n$-butylbenzamidine hydrochloride ${ }^{4}$ were prepared according to published protocols. ${ }^{1} \mathrm{H}$ and ${ }^{13} \mathrm{C}$ NMR spectra were recorded on at $200 \mathrm{MHz}$ and $50 \mathrm{MHz}$, respectively and at $25^{\circ} \mathrm{C}$ unless specified otherwise. $\mathrm{CD}$ spectra were collected at $25^{\circ} \mathrm{C}$ at $10^{-3} \mathrm{M}$ and $10^{-4} \mathrm{M}$ concentrations for $\mathbf{1}_{\mathrm{nBu}}$ and $\mathbf{2}_{\mathrm{nBu}}$, respectively, in spectrograde chloroform filtered through basic alumina, using $2 \mathrm{~nm}$ increments and $2 \mathrm{~s}$ integration time. TEM measurements were conducted on EM 400 mesh grids covered by a carbon film. Solutions of defined concentration were adsorbed for a few seconds and the excess liquid removed by absorption using Whatman filter paper. After air drying, the grids were rotated and metallized with platinum on tungsten $\left(13^{\circ}\right.$ angle $)$. The grids were observed under a tension of $120 \mathrm{kV}$. Powder diffraction samples were prepared from the synthesized solids (isolated by evaporation and washing) and plated in sealed tubes or between mica plates and submitted to X-rays generated by a copper anti-cathode $(35 \mathrm{kV}, 35 \mathrm{~mA})$.

4-tert-Butylbenzamidine hydrochloride: Same protocol as for the linear butyl chain, ${ }^{4}$ starting from commercial 4-tert-butylbenzonitrile (80\%). ${ }^{1} \mathrm{H}$ NMR (DMSO-d 6 ): 9.5 and 9.3 (br s, 4H), $7.85\left(\mathrm{~d},{ }^{3} J=8.6 \mathrm{~Hz}, 2 \mathrm{H}\right), 7.62\left(\mathrm{~d},{ }^{3} J=8.6 \mathrm{~Hz}, 2 \mathrm{H}\right), 1.30(\mathrm{~s}, 9 \mathrm{H}) .{ }^{13} \mathrm{C}$ NMR (DMSO-d 6 ): 165.4, $156.9,127.9,125.7,124.9,34.8,30.6 . \mathrm{mp}=155$ (dec.). $\mathrm{FAB}+: 177.2\left(\mathrm{MH}^{+}\right) . \mathrm{EA}: \mathrm{C}_{11} \mathrm{H}_{17} \mathrm{~N}_{2} \mathrm{Cl}$ (212.72), calc: $\% \mathrm{C}=62.11, \% \mathrm{H}=8.06, \% \mathrm{~N}=13.17$, obs: $\% \mathrm{C}=62.20, \% \mathrm{H}=7.92, \% \mathrm{~N}=13.14$.

4-Benzyloxybenzamidine hydrochloride: Same protocol as for the linear butyl chain, ${ }^{4}$ starting from $15.0 \mathrm{~g}$ of 4-benzyloxybenzonitrile. The corresponding imidate $\left(1^{\text {st }}\right.$ step) was left to stand for 16h (yield: 84\%). ${ }^{1} \mathrm{H}$ NMR (DMSO-d $)$ ): 9.1 (br s, 4H), 7.87 (d, ${ }^{3} J=8.8 \mathrm{~Hz}, 2 \mathrm{H}$ ), 7.3-7.5 (m, 5H), 7.27 (d, $\left.{ }^{3} J=8.8 \mathrm{~Hz}, 2 \mathrm{H}\right), 5.28$ (s, 2H). ${ }^{13} \mathrm{C}$ NMR (DMSO-d 6 ): 165.7, 163.5, 137.2, 131.1, 129.4, 129.0, 128.7, 120.6, 116.1, 70.5. $\mathrm{mp}=207^{\circ} \mathrm{C} . \mathrm{FAB}+: 227.0\left(\mathrm{MH}^{+}\right) . \mathrm{EA}:$ $\mathrm{C}_{14} \mathrm{H}_{15} \mathrm{~N}_{2} \mathrm{OCl} \cdot 0.16 \mathrm{HCl}(262.73)$; calc: $\% \mathrm{C}=62.61, \% \mathrm{H}=5.69, \% \mathrm{~N}=10.43, \% \mathrm{O}=5.96, \% \mathrm{Cl}=$ 15.31, obs: $\% \mathrm{C}=62.61, \% \mathrm{H}=5.83, \% \mathrm{~N}=10.60$.

4-Dodecyloxybenzamidine hydrochloride: Same protocol as for the linear butyl chain, ${ }^{4}$ starting from $7.00 \mathrm{~g}$ of the above mentioned mixture, yielding $3.65 \mathrm{~g}$ of a white powder $(45 \%)$. ${ }^{1} \mathrm{H} \mathrm{NMR}$ (DMSO-d $)_{6}$ : 8.6-9.4 (br s, 2H), $7.83\left(\mathrm{~d},{ }^{3} J=8.9 \mathrm{~Hz}, 2 \mathrm{H}\right), 7.14\left(\mathrm{~d},{ }^{3} J=8.9 \mathrm{~Hz}, 2 \mathrm{H}\right), 4.08\left(\mathrm{t},{ }^{3} J=\right.$ $6.4 \mathrm{~Hz}, 2 \mathrm{H}), 1.73(\mathrm{~m}, 2 \mathrm{H}), 1.1-1.5(\mathrm{~m}, 20 \mathrm{H}), 0.85\left(\mathrm{t},{ }^{3} J=6.5 \mathrm{~Hz}, 3 \mathrm{H}\right) .{ }^{13} \mathrm{C}$ NMR (DMSO-d 6 ): 164.6, 163.0, 130.1, 119.1, 114.7, 68.0, 31.2, 28.9, 28.6, 28.3, 25.3, 22.0, 13.8. 
2-(4-tert-Butylphenyl)-4,6-dihydroxypyrimidine: Same protocol as for the linear butyl chain ${ }^{5}$ (yield: 92\%). ${ }^{1} \mathrm{H}$ NMR (DMSO-d $\left.{ }_{6}\right): 8.8$ (br s, 1H), $8.05\left(\mathrm{~d},{ }^{3} J=8.5 \mathrm{~Hz}, 2 \mathrm{H}\right), 7.54\left(\mathrm{~d},{ }^{3} \mathrm{~J}=8.6 \mathrm{~Hz}\right.$, 2H), $5.32(\mathrm{~s}, 1 \mathrm{H}), 1.31(\mathrm{~s}, 9 \mathrm{H}) .{ }^{13} \mathrm{C}$ NMR (DMSO-d $)$ : 167.3, 157.2, 154.6, 129.3, 127.5, 125.3, 88.1, 34.6, 30.8. $\mathrm{mp}>260^{\circ} \mathrm{C}$ (dec.). $\mathrm{FAB}+245.2\left(\mathrm{MH}^{+}\right) . \mathrm{EA}: \mathrm{C}_{14} \mathrm{H}_{16} \mathrm{~N}_{2} \mathrm{O}_{2}(244.29)$, calc: $\% \mathrm{C}=$ $68.83, \% \mathrm{H}=6.60, \% \mathrm{~N}=11.47, \% \mathrm{O}=13.10$, obs: $\% \mathrm{C}=68.79, \% \mathrm{H}=6.60, \% \mathrm{~N}=11.33$.

2-(4-Benzyloxyphenyl)-4,6-dihydroxypyrimidine: Same protocol as for the linear butyl chain ${ }^{5}$ (yield: 87\%). ${ }^{1} \mathrm{H}$ NMR (DMSO-d $\left.\mathrm{d}_{6}\right) 11.8$ (br s, 2H), 8.11 (d, $\left.{ }^{3} J=8.8 \mathrm{~Hz} ; 2 \mathrm{H}\right), 7.1-7.5$ (m, 5H), $7.16\left(\mathrm{~d},{ }^{3} J=8.8 \mathrm{~Hz}, 2 \mathrm{H}\right), 5.32(\mathrm{~s}, 1 \mathrm{H}), 5.21(\mathrm{~s}, 2 \mathrm{H}) .{ }^{13} \mathrm{C}$ NMR $\left(\mathrm{DMSO}-\mathrm{d}_{6}\right): 168.3,162.1,157.9$, $137.5,130.5,129.4,128.9,128.7,125.3,115.7,88.7,70.4 . \mathrm{mp}>260^{\circ} \mathrm{C} . \mathrm{FAB}+: 295.1\left(\mathrm{MH}^{+}\right)$. EA: $\mathrm{C}_{17} \mathrm{H}_{14} \mathrm{~N}_{2} \mathrm{O}_{3} \cdot 0.57 \mathrm{HCl}(294.30)$, calc: $\% \mathrm{C}=64.80, \% \mathrm{H}=4.66, \% \mathrm{~N}=8.89, \% \mathrm{O}=15.23$; obs: $\% \mathrm{C}=64.79, \% \mathrm{H}=4.38, \% \mathrm{~N}=8.92$.

2-(4-Dodecyloxyphenyl)-4,6-dihydroxypyrimidine: Same protocol as for the linear butyl chain $^{5}$ (yield: 97 \%). ${ }^{1} \mathrm{H}$ NMR (DMSO-d $): 11.8$ (br s, 2H), 8.09 (d, $\left.{ }^{3} J=8.8 \mathrm{~Hz}, 2 \mathrm{H}\right), 7.05$ (d, ${ }^{3} J$ $=8.8 \mathrm{~Hz}, 2 \mathrm{H}), 5.28(\mathrm{~s}, 1 \mathrm{H}), 4.06\left(\mathrm{t},{ }^{3} J=6.3 \mathrm{~Hz}, 2 \mathrm{H}\right), 1.72$ (quint., $\left.{ }^{3} J=6.6 \mathrm{~Hz}, 2 \mathrm{H}, \mathrm{H}_{\beta}\right), 1.1-1.5$ $(\mathrm{m}, 20 \mathrm{H}), 0.87\left(\mathrm{t},{ }^{3} J=6.7 \mathrm{~Hz}, 3 \mathrm{H}\right) .{ }^{13} \mathrm{C}$ NMR (DMSO-d 6 ): 162.5, 157.8, 130.4, 124.9, 115.3, 87.5, 68.7, 32.2, 29.9, 29.6, 29.4, 26.3, 23.0, 14.8. mp > 260 $\mathrm{C}$ (dec.). EI: $372.3\left(\mathrm{M}^{-}\right), 204.2((\mathrm{M}-$ $\left.\left.\mathrm{C}_{12} \mathrm{H}_{25}\right)^{-}\right)$. $\mathrm{EA}: \mathrm{C}_{22} \mathrm{H}_{32} \mathrm{~N}_{2} \mathrm{O}_{3} \cdot 0.44 \mathrm{HCl}(372.50)$, calc: $\% \mathrm{C}=68.01, \% \mathrm{H}=8.42, \% \mathrm{~N}=7.21, \% \mathrm{O}=$ 12.39, obs.: $\% \mathrm{C}=68.02, \% \mathrm{H}=8.05, \% \mathrm{~N}=7.48$.

2-(4-tert-Butylphenyl)-4,6-dichloropyrimidine: Same protocol as for the linear butyl chain ${ }^{5}$ (yield: 92\%). ${ }^{1} \mathrm{H}$ NMR $\left(\mathrm{CDCl}_{3}\right): 8.35\left(\mathrm{~d},{ }^{3} J=8.6 \mathrm{~Hz}, 2 \mathrm{H}\right), 7.51\left(\mathrm{~d},{ }^{3} J=8.7 \mathrm{~Hz}, 2 \mathrm{H}\right), 7.22$ (s, 1H), 1.37 (s, 9H). ${ }^{13} \mathrm{C}$ NMR $\left(\mathrm{CDCl}_{3}\right)$ : 165.9, 162.0, 156.0, 132.3, 128.8, 125.8, 118.4, 35.1, 31.3. $\mathrm{R}_{\mathrm{f}}\left(\mathrm{SiO}_{2}\right.$, hexane $\left./ \mathrm{CH}_{2} \mathrm{Cl}_{2} 2.0: 0.3\right)=0.47 . \mathrm{mp}=93^{\circ} \mathrm{C} . \mathrm{FAB}+: 281.1\left(\mathrm{MH}^{+}\right) . \mathrm{EA}: \mathrm{C}_{14} \mathrm{H}_{14} \mathrm{~N}_{2} \mathrm{Cl}_{2}$ (281.18), calc: $\% \mathrm{C}=59.96, \% \mathrm{H}=5.04, \% \mathrm{~N}=10.05, \% \mathrm{Cl}=25.31$, obs: $\% \mathrm{C}=59.91, \% \mathrm{H}=$ $5.02, \% \mathrm{~N}=9.85$.

2-(4-Benzyloxyphenyl)-4,6-dichloropyrimidine: Same protocol as for the linear butyl chain ${ }^{5}$ (yield: $80 \%$ \%). ${ }^{1} \mathrm{H}$ NMR $\left(\mathrm{CDCl}_{3}\right): 8.39\left(\mathrm{~d},{ }^{3} \mathrm{~J}=9.0 \mathrm{~Hz}, 2 \mathrm{H}\right), 7.3-7.5(\mathrm{~m}, 5 \mathrm{H}), 7.18(\mathrm{~s}, 1 \mathrm{H}), 7.06$ (d, $\left.{ }^{3} J=9.0 \mathrm{~Hz}, 2 \mathrm{H}\right), 5.15(\mathrm{~s}, 2 \mathrm{H}) .{ }^{13} \mathrm{C} \mathrm{NMR}\left(\mathrm{CDCl}_{3}\right): 165.5,162.3,161.8,136.4,130.8,128.6$, 128.2, 127.8, 127.5, 117.7, 114.9, 70.1. $\mathrm{R}_{\mathrm{f}}\left(\mathrm{SiO}_{2}\right.$, hexane $\left./ \mathrm{CH}_{2} \mathrm{Cl}_{2} 2.0: 1.0\right)=0.35 . \mathrm{mp}=117^{\circ} \mathrm{C}$. EI: $330.2\left(\mathrm{M}^{-}\right)$. EA: $\mathrm{C}_{17} \mathrm{H}_{12} \mathrm{~N}_{2} \mathrm{OCl}_{2}(331.20)$, calc: $\% \mathrm{C}=61.65, \% \mathrm{H}=3.65, \% \mathrm{~N}=8.46$, obs: \%C $=61.59, \% \mathrm{H}=3.61, \% \mathrm{~N}=8.94$. 
2-(4-Dodecyloxyphenyl)-4,6-dichloropyrimidine: Same protocol as for the linear butyl chain, ${ }^{5}$ using hexane $/ \mathrm{CH}_{2} \mathrm{Cl}_{2}$ 4.0:0.5 to $3.0: 1.0$ as an eluent for the purification by column chromatography (yield: 93 \%). ${ }^{1} \mathrm{H} \mathrm{NMR}\left(\mathrm{CDCl}_{3}\right): 8.37\left(\mathrm{~d},{ }^{3} \mathrm{~J}=8.9 \mathrm{~Hz}, 2 \mathrm{H}\right), 7.17(\mathrm{~s}, 1 \mathrm{H}), 6.96$ (d, $\left.{ }^{3} J=8.9 \mathrm{~Hz}, 2 \mathrm{H}\right), 4.03\left(\mathrm{t},{ }^{3} J=6.5 \mathrm{~Hz}, 2 \mathrm{H}\right), 1.81$ (quint., $\left.{ }^{3} J=7 \mathrm{~Hz}, 2 \mathrm{H}\right), 1.1-1.6(\mathrm{~m}, 20 \mathrm{H}), 0.88$ (t, $\left.{ }^{3} J=6 \mathrm{~Hz}, 3 \mathrm{H}\right) .{ }^{13} \mathrm{C}$ NMR $\left(\mathrm{CDCl}_{3}\right): 165.9,163.1,162.2,131.1,127.6,117.9,114.9,68.6,32.3$, 30.0, 29.9, 29.7, 26.3, 23.0, 14.4. $\mathrm{R}_{\mathrm{f}}\left(\mathrm{SiO}_{2}\right.$, hexane $\left./ \mathrm{CH}_{2} \mathrm{Cl}_{2} 4.0: 0.05\right)=0.33 . \mathrm{mp}=52^{\circ} \mathrm{C}$. EI: $408.3\left(\mathrm{M}^{-}\right), 240.1\left(\left(\mathrm{M}-\mathrm{C}_{12} \mathrm{H}_{25}\right)^{-}\right) . \mathrm{EA}: \mathrm{C}_{22} \mathrm{H}_{30} \mathrm{~N}_{2} \mathrm{OCl}_{2}(409.39)$, calc: $\% \mathrm{C}=64.54, \% \mathrm{H}=7.39$, $\% \mathrm{~N}=6.84, \% \mathrm{O}=3.91, \% \mathrm{Cl}=17.32$, obs: $\% \mathrm{C}=64.60, \% \mathrm{H}=7.37, \% \mathrm{~N}=6.77$.

2-(4-tert-Butylphenyl)-4-chloro-6-ethoxyvinyl)-pyrimidine: Idem as for the linear chain; the intermediate enol ether was isolated. ${ }^{1} \mathrm{H}$ NMR $\left(\mathrm{CDCl}_{3}\right): 8.40\left(\mathrm{~d},{ }^{3} J=8.6 \mathrm{~Hz}, 2 \mathrm{H}\right), 7.49(\mathrm{~s}, 1 \mathrm{H})$, $7.51\left(\mathrm{~d},{ }^{3} J=8.6 \mathrm{~Hz}, 2 \mathrm{H}\right), 5.89\left(\mathrm{~d},{ }^{2} J=2.0 \mathrm{~Hz}, 1 \mathrm{H}\right), 4.58\left(\mathrm{~d},{ }^{2} J=2.0 \mathrm{~Hz}, 1 \mathrm{H}\right), 3.99$ (quadr., ${ }^{3} J=$ $7.0 \mathrm{~Hz}, 2 \mathrm{H}), 1.46\left(\mathrm{t},{ }^{3} J=7.0 \mathrm{~Hz}, 3 \mathrm{H}\right), 1.37$ (s, 9H). ${ }^{13} \mathrm{C} \mathrm{NMR}\left(\mathrm{CDCl}_{3}\right): 164.5,162.6,162.1$, 156.3, 154.8, 133.8, 128.9, 125.6, 113.4, 89.0, 63.9, 35.0, 31.3, 14.5. $\mathrm{R}_{\mathrm{f}}\left(\mathrm{SiO}_{2}\right.$, hexane $/ \mathrm{CH}_{2} \mathrm{Cl}_{2}$ 2.0: 0.5) $=0.28 . \mathrm{mp}=105^{\circ} \mathrm{C} . \mathrm{FAB}+: 317.3\left(\mathrm{MH}^{+}\right) . \mathrm{EA}: \mathrm{C}_{18} \mathrm{H}_{21} \mathrm{~N}_{2} \mathrm{OCl} \cdot 0.11 \mathrm{CH}_{2} \mathrm{Cl}_{2}$, calc: \%C= $66.69, \% \mathrm{H}=6.56, \% \mathrm{~N}=8.59, \% \mathrm{O}=4.91, \% \mathrm{Cl}=13.23$, obs: $\% \mathrm{C}=66.69, \% \mathrm{H}=6.79, \% \mathrm{~N}=$ 8.83 .

2-(4-Benzyloxyphenyl)-4-chloro-6-ethoxyvinyl)-pyrimidine: Idem as for the linear chain. The intermediate enol ether was isolated and recrystallized in diethyl ether/hexane (yield: $95 \%$ ). ${ }^{1} \mathrm{H}$ NMR $\left(\mathrm{CDCl}_{3}\right): 8.44\left(\mathrm{~d},{ }^{3} J=8.8 \mathrm{~Hz}, 2 \mathrm{H}\right), 7.3-7.5(\mathrm{~m}, 6 \mathrm{H}), 7.06\left(\mathrm{~d},{ }^{3} J=8.8 \mathrm{~Hz}, 2 \mathrm{H}\right), 5.86\left(\mathrm{~d},{ }^{2} J=\right.$ $1.9 \mathrm{~Hz}, 1 \mathrm{H}), 5.15$ (s, 2H), 4.57 (d, $\left.{ }^{2} J=1.8 \mathrm{~Hz}, 1 \mathrm{H}\right), 3.99$ (quadr., $\left.{ }^{3} J=7.0 \mathrm{~Hz}, 2 \mathrm{H}\right), 1.46\left(\mathrm{t},{ }^{3} J=\right.$ $7.0 \mathrm{~Hz}, 3 \mathrm{H}) .{ }^{13} \mathrm{C} \mathrm{NMR}\left(\mathrm{CDCl}_{3}\right): 164.4,162.8,162.3,161.9,156.6,137.0,130.7,129.7,129.0$, 128.4 , 127.9, 115.1, 113.2, 89.2, 70.5, 64.2, 14.8. $\mathrm{R}_{\mathrm{f}}\left(\mathrm{SiO}_{2}\right.$, hexane $/ \mathrm{CH}_{2} \mathrm{Cl}_{2}$ 2.0:1.0) $=0.45 . \mathrm{mp}=$ $119^{\circ} \mathrm{C}$ EI: $\left.366.3\left(\mathrm{M}^{-}\right), 351.2\left(\left(\mathrm{M}-\mathrm{CH}_{3}\right)^{-}\right), 322.2\left(\left(\mathrm{M}-\mathrm{OC}_{2} \mathrm{H}_{5}\right)^{-}\right)^{-}\right)$. EA: $\mathrm{C}_{21} \mathrm{H}_{19} \mathrm{~N}_{2} \mathrm{O}_{2} \mathrm{Cl}(366.84)$, calc: $\% \mathrm{C}=68.76, \% \mathrm{H}=5.22, \% \mathrm{~N}=7.64, \% \mathrm{O}=8.72, \% \mathrm{Cl}=9.66$, obs: $\% \mathrm{C}=68.46, \% \mathrm{H}=$ $5.04, \% \mathrm{~N}=8.22$.

4-Chloro-2-(4-dodecyloxyphenyl)-6-(1-ethoxyvinyl)-pyrimidine: Idem as for the linear chain; the intermediate enol ether 4-chloro-2-(4-dodecyloxyphenyl)-6-(1-ethoxyvinyl)-pyrimidine (52 $\%)$, the bis enol ether (4,6-bis-(1-ethoxyvinyl)-2-(4-dodecyloxyphenyl)-pyrimidine; $11 \%$ ) and starting 2-(4-dodecyloxyphenyl)-4,6-dichloropyrimidine (5\%) were isolated after flash chromatography on silica gel (hexane/ $\left.\mathrm{CH}_{2} \mathrm{Cl}_{2} 2: 1\right)$. ${ }^{1} \mathrm{H} \mathrm{NMR}\left(\mathrm{CDCl}_{3}\right): 8.42\left(\mathrm{~d},{ }^{3} J=9.0 \mathrm{~Hz}, 2 \mathrm{H}\right)$, $7.41(\mathrm{~s}, 1 \mathrm{H}), 6.97\left(\mathrm{~d},{ }^{3} J=9.0 \mathrm{~Hz}, 2 \mathrm{H}\right), 5.85\left(\mathrm{~d},{ }^{2} J=1.9 \mathrm{~Hz}, 1 \mathrm{H}\right), 4.56\left(\mathrm{~d},{ }^{2} J=1.9 \mathrm{~Hz}, 1 \mathrm{H}\right), 4.02$ 
(t, ${ }^{3} J=6.4 \mathrm{~Hz}, 2 \mathrm{H}$ ), 3.98 (quadr., ${ }^{3} J=6.9 \mathrm{~Hz}, 2 \mathrm{H}$ ), 1.81 (quint., ${ }^{3} J=7.6 \mathrm{~Hz}, 2 \mathrm{H}$ ), 1.46 (quadr., ${ }^{3} J$ $=7.0 \mathrm{~Hz}, 2 \mathrm{H}), 1.1-1.5(\mathrm{~m}, 20 \mathrm{H}), 0.88\left(\mathrm{t},{ }^{3} \mathrm{~J}=6.4 \mathrm{~Hz}, 3 \mathrm{H}\right) .{ }^{13} \mathrm{C} \mathrm{NMR}\left(\mathrm{CDCl}_{3}\right): 164.2,162.4$, 162.0, 156.3, 130.2, 128.8, 114.4, 112.8, 88.8, 68.2, 63.9, 31.9, 29.6, 29.4, 29.4, 29.2 22.7, 26.1, 14.4, 14.1. $\mathrm{R}_{\mathrm{f}}\left(\mathrm{SiO}_{2}\right.$, hexane $/ \mathrm{CH}_{2} \mathrm{Cl}_{2}$ 1.5:1.0) =0.50. $\mathrm{mp}=91^{\circ} \mathrm{C}$. EI: $444.3\left(\mathrm{M}^{-}\right), 415.4\left(\mathrm{M}^{-}-\right.$ $\mathrm{C}_{2} \mathrm{H}_{5}$ ). EA: $\mathrm{C}_{26} \mathrm{H}_{37} \mathrm{~N}_{2} \mathrm{O}_{2} \mathrm{Cl}(445.04)$, calc: $\% \mathrm{C}=70.17, \% \mathrm{H}=8.38, \% \mathrm{~N}=6.29, \% \mathrm{O}=7.19, \% \mathrm{Cl}$ $=7.97$, obs: $\% \mathrm{C}=70.11, \% \mathrm{H}=8.36, \% \mathrm{~N}=6.20$.

1-[2-(4-tert-Butylphenyl)-6-chloro-pyrimidin-4-yl]-ethyl ketone: The hydrolysis of the enol ether was quantitative (overall yield for monoacylation: $72 \%) .{ }^{1} \mathrm{H}$ NMR $\left(\mathrm{CDCl}_{3}\right): 8.42\left(\mathrm{~d},{ }^{3} \mathrm{~J}=\right.$ $8.7 \mathrm{~Hz}, 2 \mathrm{H}), 7.73(\mathrm{~s}, 1 \mathrm{H}), 7.54\left(\mathrm{~d},{ }^{3} J=8.7 \mathrm{~Hz}, 2 \mathrm{H}\right), 2.80(\mathrm{~s}, 3 \mathrm{H}), 1.38(\mathrm{~s}, 9 \mathrm{H}) .{ }^{13} \mathrm{C}$ NMR $\left(\mathrm{CDCl}_{3}\right): 198.7,165.9,163.5,160.5,155.7,132.9,128.6,125.9,115.2,35.1,31.3,25.9 . \mathrm{R}_{\mathrm{f}}\left(\mathrm{SiO}_{2}\right.$, hexane $\left./ \mathrm{CH}_{2} \mathrm{Cl}_{2} 2.0: 1.0\right)=0.31 . \mathrm{mp}=92^{\circ} \mathrm{C} . \mathrm{FAB}+: 289.2\left(\mathrm{MH}^{+}\right) . \mathrm{EA}: \mathrm{C}_{16} \mathrm{H}_{17} \mathrm{~N}_{2} \mathrm{OCl}(288.77)$, calc: $\% \mathrm{C}=66.55, \% \mathrm{H}=5.93, \% \mathrm{~N}=9.70, \% \mathrm{O}=5.54, \% \mathrm{Cl}=12.28$, obs: $\% \mathrm{C}=66.37, \% \mathrm{H}=$ $6.09, \% \mathrm{~N}=9.66$.

1-[2-(4-Benzyloxyphenyl)-6-chloro-pyrimidin-4-yl]-ethyl ketone: The hydrolysis of the enol ether was quantitative. ${ }^{1} \mathrm{H} \mathrm{NMR}\left(\mathrm{CDCl}_{3}\right): 8.46\left(\mathrm{~d},{ }^{3} J=9.0 \mathrm{~Hz}, 2 \mathrm{H}\right), 7.68(\mathrm{~s}, 1 \mathrm{H}), 7.3-7.5(\mathrm{~m}$, 5H), $7.09\left(\mathrm{~d},{ }^{3} \mathrm{~J}=9.0 \mathrm{~Hz}, 2 \mathrm{H}\right), 5.17$ (s, 2H), 2.79 (s, 3H). ${ }^{13} \mathrm{C} \mathrm{NMR}\left(\mathrm{CDCl}_{3}\right): 198.5,165.4,163.2$, $162.0,160.4,136.4,130.5,128.6,128.3,128.2,127.5,115.0,114.5,70.1,25.8 . \mathrm{R}_{\mathrm{f}}\left(\mathrm{SiO}_{2}\right.$, hexane $/ \mathrm{CH}_{2} \mathrm{Cl}_{2}$ 1.0:1.0) $=0.20 . \mathrm{mp}=93^{\circ} \mathrm{C}$. EI: $338.3\left(\mathrm{M}^{-}\right)$. EA: $\mathrm{C}_{19} \mathrm{H}_{15} \mathrm{~N}_{2} \mathrm{O}_{2} \mathrm{Cl}$ (338.79), calc: $\% \mathrm{C}=67.36, \% \mathrm{H}=4.46, \% \mathrm{~N}=8.27, \% \mathrm{O}=9.44, \% \mathrm{Cl}=10.46$, obs: $\% \mathrm{C}=67.40, \% \mathrm{H}=4.49$, $\% \mathrm{~N}=8.05$.

1-[2-(4-Dodecyloxyphenyl)- 6-chloropyrimidin-4-yl]-ethyl ketone: The hydrolysis was carried out in the same manner starting from $1.67 \mathrm{~g}$ of 4-chloro-2-(4-dodecyloxyphenyl)-6-(1ethoxyvinyl)-pyrimidine $(3.75 \mathrm{mmol})$ and yielding $1.34 \mathrm{~g}$ of a white solid $(88 \%)$ after flash chromatography on silica gel (hexane/ $\mathrm{CH}_{2} \mathrm{Cl}_{2} 3.0: 1.0$ to 2.0:1.0). ${ }^{1} \mathrm{H} \mathrm{NMR}\left(\mathrm{CDCl}_{3}\right): 8.41\left(\mathrm{~d},{ }^{3} \mathrm{~J}=\right.$ $8.9 \mathrm{~Hz}, 2 \mathrm{H}), 7.64(\mathrm{~s}, 1 \mathrm{H}), 6.98\left(\mathrm{~d},{ }^{3} J=8.9 \mathrm{~Hz}, 2 \mathrm{H}\right), 4.03\left(\mathrm{t},{ }^{3} J=6.5 \mathrm{~Hz}, 2 \mathrm{H}\right), 2.77$ (s, 3H), 1.82 (quint., $\left.{ }^{3} J=7.4 \mathrm{~Hz}, 2 \mathrm{H}\right), 1.1-1.7(\mathrm{~m}, 20 \mathrm{H}), 0.88$ (t, $\left.{ }^{3} J=6.2 \mathrm{~Hz}, 3 \mathrm{H}\right) .{ }^{13} \mathrm{C} \mathrm{NMR}\left(\mathrm{CDCl}_{3}\right): 198.9$, $165.9,163.5,162.8,160.7,130.8,128.3,114.9,114.7,68.6,32.3,30.0,29.8,29.5,26.4,26.2$, 23.0, 14.5. $\mathrm{R}_{\mathrm{f}}\left(\mathrm{SiO}_{2}\right.$, hexane/ $\left.\mathrm{CH}_{2} \mathrm{Cl}_{2} 2.0: 1.0\right)=0.27 . \mathrm{mp}=73^{\circ} \mathrm{C}$. EI : $416.5\left(\mathrm{M}^{-}\right), 248((\mathrm{M}-$ $\left.\left.\mathrm{C}_{12} \mathrm{H}_{25}\right)^{-}\right)$. $\mathrm{EA}: \mathrm{C}_{24} \mathrm{H}_{33} \mathrm{~N}_{2} \mathrm{O}_{2} \mathrm{Cl}(416.98)$, calc: $\% \mathrm{C}=69.13, \% \mathrm{H}=7.98, \% \mathrm{~N}=6.72, \% \mathrm{O}=7.67$, $\% \mathrm{Cl}=8.5$, obs: $\% \mathrm{C}=69.13, \% \mathrm{H}=8.05, \% \mathrm{~N}=6.62$. 
1-[2-(4-tert-Butylphenyl)-6-(1-ethoxyvinyl)-pyrimidin-4-yl]-ethyl ketone: Idem as for the linear chain (yield: $82 \%) .{ }^{1} \mathrm{H}$ NMR $\left(\mathrm{CDCl}_{3}\right): 8.47\left(\mathrm{~d},{ }^{3} J=8.3 \mathrm{~Hz}, 2 \mathrm{H}\right), 8.03(\mathrm{~s}, 1 \mathrm{H}), 7.54\left(\mathrm{~d},{ }^{3} J\right.$ $=8.4 \mathrm{~Hz}, 2 \mathrm{H}), 5.89\left(\mathrm{~d},{ }^{2} J=2.0 \mathrm{~Hz}, 1 \mathrm{H}\right), 4.57\left(\mathrm{~d},{ }^{2} J=2.0 \mathrm{~Hz}, 1 \mathrm{H}\right), 4.01$ (quadr., ${ }^{3} J=7.0 \mathrm{~Hz}, 2 \mathrm{H}$ ), $2.82(\mathrm{~s}, 3 \mathrm{H}), 1.48\left(\mathrm{t},{ }^{3} \mathrm{~J}=7.0 \mathrm{~Hz}, 3 \mathrm{H}\right), 1.39$ (s, 9H). ${ }^{13} \mathrm{C} \mathrm{NMR}\left(\mathrm{CDCl}_{3}\right): 200.4,164.1,162.8$, $160.5,157.0,154.5,134.5,128.2,125.6,109.1,88.2,64.0,35.0,31.3,25.9,14.6 . \mathrm{R}_{\mathrm{f}}\left(\mathrm{SiO}_{2}\right.$, hexane $\left./ \mathrm{CH}_{2} \mathrm{Cl}_{2} 2.0: 1.0\right)=0.24 . \mathrm{mp}=109^{\circ} \mathrm{C}$ (dec.). $\mathrm{FAB}+: 325.3\left(\mathrm{MH}^{+}\right) . \mathrm{EA}: \mathrm{C}_{20} \mathrm{H}_{24} \mathrm{~N}_{2} \mathrm{O}_{2} \cdot 0.06$ $\mathrm{CH}_{2} \mathrm{Cl}_{2}$ (324.44), calc: $\% \mathrm{C}=73.12, \% \mathrm{H}=7.38, \% \mathrm{~N}=8.50, \% \mathrm{O}=9.70$, obs: $\% \mathrm{C}=73.22, \% \mathrm{H}=$ $7.37, \% \mathrm{~N}=8.58$.

1-[2-(4-Benzyloxyphenyl)-6-(1-ethoxyvinyl)-pyrimidin-4-yl]-ethyl ketone: Idem as for the linear chain, using a 1.0:1.0 hexane/ $\mathrm{CH}_{2} \mathrm{Cl}_{2}$ mixture as an eluent for flash chromatography on silica gel (yield: $86 \%$ \%). ${ }^{1} \mathrm{H}$ NMR $\left(\mathrm{CDCl}_{3}\right): 8.53\left(\mathrm{~d},{ }^{3} J=8.8 \mathrm{~Hz}, 2 \mathrm{H}\right), 7.99(\mathrm{~s}, 1 \mathrm{H}), 7.3-7.5(\mathrm{~m}$, $5 \mathrm{H}), 7.10\left(\mathrm{~d},{ }^{3} J=8.8 \mathrm{~Hz}, 2 \mathrm{H}\right), 5.86\left(\mathrm{~d},{ }^{2} J=1.8 \mathrm{~Hz}, 1 \mathrm{H}\right), 5.17(\mathrm{~s}, 2 \mathrm{H}), 4.56\left(\mathrm{~d},{ }^{2} J=1.6 \mathrm{~Hz}, 1 \mathrm{H}\right)$, 4.01 (quadr., $\left.{ }^{3} J=7.0 \mathrm{~Hz}, 2 \mathrm{H}\right), 2.80$ (s, 3H), 1.47 (t, $\left.{ }^{3} J=7.0 \mathrm{~Hz}, 3 \mathrm{H}\right) .{ }^{13} \mathrm{C} \mathrm{NMR}\left(\mathrm{CDCl}_{3}\right): 200.4$, $163.7,162.7,161.4,160.4,157.0,136.7,130.1,128.7,128.1,127.5,114.9,108.6,88.1,70.1$, 63.9, 25.8, 14.5. $\mathrm{R}_{\mathrm{f}}\left(\mathrm{SiO}_{2}\right.$, hexane $/ \mathrm{CH}_{2} \mathrm{Cl}_{2}$ 1.0:1.0) =0.30. $\mathrm{mp}=126^{\circ} \mathrm{C}$. EI: $374.3\left(\mathrm{M}^{\circ}\right), 359.3$ $\left(\left(\mathrm{M}-\mathrm{CH}_{3}\right)^{-}\right), 330.2\left(\left(\mathrm{M}-\mathrm{OC}_{2} \mathrm{H}_{5}\right)^{-}\right)$. EA: $\mathrm{C}_{20} \mathrm{H}_{24} \mathrm{~N}_{2} \mathrm{O}_{2} \cdot 0.06 \mathrm{CH}_{2} \mathrm{Cl}_{2}(374.43)$, calc: $\% \mathrm{C}=73.20, \% \mathrm{H}$ $=7.38, \% \mathrm{~N}=8.51, \% \mathrm{O}=9.70, o b s: \% \mathrm{C}=73.22, \% \mathrm{H}=7.37, \% \mathrm{~N}=8.58$.

1-[2-(4-Dodecyloxyphenyl)-6-(1-ethoxyvinyl)-pyrimidin-4-yl]-ethyl ketone: Idem as for the linear chain, using hexane/ $\mathrm{CH}_{2} \mathrm{Cl}_{2}(2.0: 1.0$ to 2.5:2.0) as an eluent for flash chromatography on silica gel (yield: $90 \%) .{ }^{1} \mathrm{H}$ NMR $\left(\mathrm{CDCl}_{3}\right): 8.51\left(\mathrm{~d},{ }^{3} J=8.9 \mathrm{~Hz}, 2 \mathrm{H}\right), 7.98(\mathrm{~s}, 1 \mathrm{H}), 7.01\left(\mathrm{~d},{ }^{3} J=\right.$ $8.9 \mathrm{~Hz}, 2 \mathrm{H}), 5.86\left(\mathrm{~d},{ }^{2} J=1.9 \mathrm{~Hz}, 1 \mathrm{H}\right), 4.55\left(\mathrm{~d},{ }^{2} J=1.8 \mathrm{~Hz}, 1 \mathrm{H}\right), 4.05$ (quadr., ${ }^{3} J=6.5 \mathrm{~Hz}, 2 \mathrm{H}$ ), 3.99 (t, $\left.{ }^{3} J=6 \mathrm{~Hz}, 2 \mathrm{H}\right), 2.81$ (s, 3H), 1.83 (quint., $\left.{ }^{3} J=7.6 \mathrm{~Hz}, 2 \mathrm{H}\right), 1.47$ (t, $\left.{ }^{3} J=7.0 \mathrm{~Hz}, 3 \mathrm{H}\right), 1.2-$ $1.6(\mathrm{~m}, 20 \mathrm{H}), 0.88\left(\mathrm{t},{ }^{3} \mathrm{~J}=6.4 \mathrm{~Hz}, 3 \mathrm{H}\right) .{ }^{13} \mathrm{C} \mathrm{NMR}\left(\mathrm{CDCl}_{3}\right): 200.7,164.1,163.0,162.1,160.7$, 157.3, 130.3, 129.9, 114.8, 108.8, 88.3, 68.5, 64.2, 32.3, 30.0, 29.8, 29.6, 26.4, 26.1, 23.0, 14.8, 14.5. $\mathrm{R}_{\mathrm{f}}\left(\mathrm{SiO}_{2}\right.$, hexane $\left./ \mathrm{CH}_{2} \mathrm{Cl}_{2} 2.0: 1.0\right)=0.36 . \mathrm{mp}=81^{\circ} \mathrm{C}$. EI: 452.5(M-), $437.4\left(\left(\mathrm{M}^{-} \mathrm{CH}_{3}\right)^{-}\right)$, $269.2\left(\left(\mathrm{M}-\mathrm{CH}_{3}-\mathrm{C}_{12} \mathrm{H}_{25}\right)^{-}\right), \quad 253 \quad\left(\left(\mathrm{M}-\mathrm{C}_{12} \mathrm{H}_{25}-\mathrm{C}_{2} \mathrm{H}_{5}\right)^{-}\right), \quad 240.0 \quad\left(\left(\mathrm{M}-\mathrm{CH}_{3}-\mathrm{C}_{12} \mathrm{H}_{25}-\mathrm{CO}\right)^{-}\right) . \quad$ EA: $\mathrm{C}_{28} \mathrm{H}_{40} \mathrm{~N}_{2} \mathrm{O}_{3} \cdot 0.06 \mathrm{CH}_{2} \mathrm{Cl}_{2}$ (452.63), calc: $\% \mathrm{C}=73.63, \% \mathrm{H}=8.83, \% \mathrm{~N}=6.07, \% \mathrm{O}=10.49$, obs: $\% \mathrm{C}=73.63, \% \mathrm{H}=8.95, \% \mathrm{~N}=6.12$.

1-[6-Acetyl-2-(4-tert-butylphenyl)-pyrimidin-4-yl]-ethyl ketone: Idem as for the linear chain (overall yield, 2 steps: 95 \%). ${ }^{1} \mathrm{H}$ NMR $\left(\mathrm{CDCl}_{3}\right): 8.50\left(\mathrm{~d},{ }^{3} J=8.6 \mathrm{~Hz}, 2 \mathrm{H}\right), 8.25(\mathrm{~s}, 1 \mathrm{H}), 7.58$ (d, $\left.{ }^{3} J=8.6 \mathrm{~Hz}, 2 \mathrm{H}\right), 2.83(\mathrm{~s}, 6 \mathrm{H}), 1.40(\mathrm{~s}, 9 \mathrm{H}) .{ }^{13} \mathrm{C} \mathrm{NMR}\left(\mathrm{CDCl}_{3}\right): 199.2,165.4,161.4,155.4$, 
133.6, 128.4, 125.9, 110.5, 35.1, 31.3, 25.8. $\mathrm{R}_{\mathrm{f}}\left(\mathrm{SiO}_{2}\right.$, hexane $\left./ \mathrm{CH}_{2} \mathrm{Cl}_{2} 1.0: 2.0\right)=0.30 . \mathrm{mp}=$ 136 ${ }^{\circ} \mathrm{C} . \mathrm{FAB}+: 297.4\left(\mathrm{MH}^{+}\right)$. EA: $\mathrm{C}_{18} \mathrm{H}_{20} \mathrm{~N}_{2} \mathrm{O}_{2}(296.36)$ : calc: $\% \mathrm{C}=72.94, \% \mathrm{H}=6.81, \% \mathrm{~N}=$ $9.46, \% \mathrm{O}=10.8$, obs: $\% \mathrm{C}=72.84, \% \mathrm{H}=6.79, \% \mathrm{~N}=9.61$.

1-[6-Acetyl-2-(4-benzyloxylphenyl)-pyrimidin-4-yl]-ethyl ketone: Idem as for the linear chain (global yield: $45 \%$ ). ${ }^{1} \mathrm{H}$ NMR $\left(\mathrm{CDCl}_{3}\right): 8.54\left(\mathrm{~d},{ }^{3} \mathrm{~J}=8.9 \mathrm{~Hz}, 2 \mathrm{H}\right), 8.21(\mathrm{~s}, 1 \mathrm{H}), 7.3-7.5(\mathrm{~m}, 5 \mathrm{H})$, $7.13\left(\mathrm{~d},{ }^{3} \mathrm{~J}=8.9 \mathrm{~Hz}, 2 \mathrm{H}\right), 5.19$ (s, 2H), $2.82(\mathrm{~s}, 6 \mathrm{H}) .{ }^{13} \mathrm{C} \mathrm{NMR}\left(\mathrm{CDCl}_{3}\right): 199.2,165.0,161.8$, $161.3,136.5,130.2,129.1,128.7,128.2,127.5,115.1,109.9,70.1,25.7 . \mathrm{R}_{\mathrm{f}}\left(\mathrm{SiO}_{2}\right.$, hexane $/ \mathrm{CH}_{2} \mathrm{Cl}_{2}$ 1.0:2.0) $=0.25 . \mathrm{mp}=144^{\circ} \mathrm{C}$. EI: $346.3\left(\mathrm{M}^{-}\right)$. EA: $\mathrm{C}_{18} \mathrm{H}_{20} \mathrm{~N}_{2} \mathrm{O}_{2} \cdot 0.022 \mathrm{CH}_{2} \mathrm{Cl}_{2}$ : calc: $\% \mathrm{C}=72.50, \% \mathrm{H}=5.22, \% \mathrm{~N}=8.04, \% \mathrm{O}=13.78$, obs: $\% \mathrm{C}=72.50, \% \mathrm{H}=5.15, \% \mathrm{~N}=$ 8.04 .

4,6-Bis-(2-amino-3-formylpyridyl)-2-(4-tert-butylphenyl)-pyrimidine ( $\left.3_{\text {tertBu }}\right)$ : Idem as for the linear chain ${ }^{29}$ (yield: $70 \%$ \%). ${ }^{1} \mathrm{H}$ NMR $\left(\mathrm{CDCl}_{3}\right)$ : 9.99 (s, 2H), 9.11 (s, $\left.1 \mathrm{H}\right), 8.60\left(\mathrm{~d},{ }^{3} J=8.5 \mathrm{~Hz}\right.$, $2 \mathrm{H}), 8.16\left(\mathrm{~d},{ }^{3} J=7.4 \mathrm{~Hz}, 2 \mathrm{H}\right), 8.01\left(\mathrm{~d},{ }^{3} J=7.6 \mathrm{~Hz}, 2 \mathrm{H}\right), 7.59\left(\mathrm{~d},{ }^{3} J=8.5 \mathrm{~Hz}, 2 \mathrm{H}\right), 6.86$ (br s, 4H), 1.42 (s, 9H). ${ }^{13}$ C NMR (DMSO-d ${ }_{6}$ ): 193.4, 163.3, 163.0, 157.8, 157.0, 153.9, 146.2, 134.1, 127.9, 125.5, 114.6, 111.8, 110.0, 34.6, 30.9. $\mathrm{R}_{\mathrm{f}}\left(\mathrm{SiO}_{2}, \mathrm{CH}_{2} \mathrm{Cl}_{2} / \mathrm{EtOAc} 2.0: 0.3\right)=0.46 . \mathrm{mp}>$ $260^{\circ} \mathrm{C}$ (dec.). $\mathrm{FAB}+: 453.0\left(\mathrm{MH}^{+}\right) . \mathrm{EA}: \mathrm{C}_{26} \mathrm{H}_{24} \mathrm{~N}_{6} \mathrm{O}_{2} \cdot 0.37$ hexane (452.20), calc: $\% \mathrm{C}=69.97$, $\% \mathrm{H}=6.07, \% \mathrm{~N}=17.35, \% \mathrm{O}=6.61, o b s: \% \mathrm{C}=69.97, \% \mathrm{H}=6.07, \% \mathrm{~N}=16.53$.

4,6-Bis-(2-amino-3-formylpyridyl)-2-(4-benzyloxylphenyl)-pyrimidine ( $\left.3_{\mathrm{OBz}}\right)$ : Idem as for the linear chain ${ }^{29}$. After $5 \mathrm{~h}$ hydrolysis and an identical work-up, both the O-benzyl substituted ( $\mathbf{3}_{\mathbf{O B z}}$, $32 \%)$ and free $\mathrm{OH}\left(\mathbf{3}_{\mathbf{O H}}, 23 \%\right)$ species were isolated by flash chromatography on silica gel $\left(\mathrm{CH}_{2} \mathrm{Cl}_{2} / \mathrm{EtOAc} 2.0: 1.0\right.$ to 0.5:1.0). 3 $\mathbf{3}_{\mathbf{\text { ozz}}}:{ }^{1} \mathrm{H}$ NMR $\left(\mathrm{CDCl}_{3}\right): 9.99$ (s, $\left.2 \mathrm{H}\right), 8.91$ (s, 1H), $8.45\left(\mathrm{~d},{ }^{3} \mathrm{~J}\right.$ $=8.2 \mathrm{~Hz}, 2 \mathrm{H}), 8.29\left(\mathrm{~d},{ }^{3} J=7.9 \mathrm{~Hz}, 2 \mathrm{H}\right), 8.04\left(\mathrm{~d},{ }^{3} J=8.2 \mathrm{~Hz}, 2 \mathrm{H}\right), 7.76(\mathrm{br} \mathrm{s}, 4 \mathrm{H}), 7.3-7.5(\mathrm{~m}$, $5 \mathrm{H}$ ), $7.22\left(\mathrm{~d},{ }^{3} J=8.5 \mathrm{~Hz}, 2 \mathrm{H}\right), 5.23$ (s, $\left.2 \mathrm{H}\right) .{ }^{13} \mathrm{C}$ NMR (DMSO-d 6 ): 193.6, 163.2, 160.9, 157.9, $157.3,154.6,146.1,136.7,129.8,128.5,127.9,114.9,114.5,110.1,69.4 . \mathrm{R}_{\mathrm{f}}\left(\mathrm{SiO}_{2}\right.$, $\mathrm{CH}_{2} \mathrm{Cl}_{2}$ /acetone 3.0:0.2) $=0.60 . \mathrm{mp}=203^{\circ} \mathrm{C}$ (dec.). $\mathrm{FAB}+$ : $503.2\left(\mathrm{MH}^{+}\right) . \mathrm{EA}: \mathrm{C}_{26} \mathrm{H}_{24} \mathrm{~N}_{6} \mathrm{O}_{2} \cdot 0.36$ EtOAc, calc: $\% \mathrm{C}=68.44, \% \mathrm{H}=4.69, \% \mathrm{~N}=15.73, \% \mathrm{O}=11.14$, obs: $\% \mathrm{C}=68.38, \% \mathrm{H}=4.64$, $\% \mathrm{~N}=15.73 .3_{\text {он }}:{ }^{1} \mathrm{H}$ NMR (DMSO-d 6 ): $9.99(\mathrm{~s}, 2 \mathrm{H}), 8.89(\mathrm{~s}, 1 \mathrm{H}), 8.51\left(\mathrm{~d},{ }^{3} J=8.7 \mathrm{~Hz}, 2 \mathrm{H}\right)$, $8.30\left(\mathrm{~d},{ }^{3} \mathrm{~J}=7.8 \mathrm{~Hz}, 2 \mathrm{H}\right), 8.03$ (d, $\left.{ }^{3} \mathrm{~J}=7.8 \mathrm{~Hz}, 2 \mathrm{H}\right), 7.73$ (br s, $\left.4 \mathrm{H}\right), 6.97\left(\mathrm{~d},{ }^{3} \mathrm{~J}=8.7 \mathrm{~Hz}, 2 \mathrm{H}\right) .{ }^{13} \mathrm{C}$ NMR (DMSO-d ${ }_{6}$ ): 193.5, 163.5, 163.1, 160.4, 157.9, 157.4, 146.0, 129.9, 128.4, 127.7, 115.5, 114.5, 110.0. $\mathrm{R}_{\mathrm{f}}\left(\mathrm{SiO}_{2}, \mathrm{CH}_{2} \mathrm{Cl}_{2} /\right.$ acetone 3.0:0.2) $=0.21 . \mathrm{mp}=235^{\circ} \mathrm{C}$ (dec.).

\section{1-[2-(4-tert-Butylphenyl)-6-[1,8]naphthyridin-2-yl-pyrimidin-4-yl]-ethyl ketone}


( $\left.\mathbf{4}_{\text {tertBu }}\right)$ : same protocol as for the linear butyl chain. ${ }^{1} \mathrm{H}$ NMR $\left(\mathrm{CDCl}_{3}\right): 9.23\left(\mathrm{dd},{ }^{3} J=4.2 \mathrm{~Hz},{ }^{4} \mathrm{~J}=\right.$ $1.9 \mathrm{~Hz}, 1 \mathrm{H}), 9.18(\mathrm{~s}, 1 \mathrm{H}), 8.95\left(\mathrm{~d},{ }^{3} J=8.5 \mathrm{~Hz}, 1 \mathrm{H}\right), 8.61\left(\mathrm{~d},{ }^{3} J=8.6 \mathrm{~Hz}, 2 \mathrm{H}\right), 8.44\left(\mathrm{~d},{ }^{3} J=8.4\right.$ $\mathrm{Hz}, 1 \mathrm{H}), 8.31\left(\mathrm{dd},{ }^{3} J=8.2 \mathrm{~Hz},{ }^{4} J=1.9 \mathrm{~Hz}, 1 \mathrm{H}\right), 7.5-7.7(\mathrm{~m}, 3 \mathrm{H}), 2.87$ (s, 3H), $1.41(\mathrm{~s}, 9 \mathrm{H}) .{ }^{13} \mathrm{C}$ NMR $\left(\mathrm{CDCl}_{3}\right)$ : 199.9, 165.0, 164.9, 162.2, 157.6, 156.0, 155.1, 154.7, 138.7, 137.3, 134.6, 128.6, 126.0, 124.2, 123.3, 120.4, 112.5, 35.3, 31.6, 26.3. $\mathrm{R}_{\mathrm{f}}\left(\mathrm{SiO}_{2}, \mathrm{CH}_{2} \mathrm{Cl}_{2} /\right.$ EtOAc 4.0:1.0) $=$ 0.29. $\mathrm{mp}=218^{\circ} \mathrm{C}$ (dec.). $\mathrm{FAB}+: 383.1\left(\mathrm{MH}^{+}\right)$. $\mathrm{EA}: \mathrm{C}_{24} \mathrm{H}_{22} \mathrm{~N}_{4} \mathrm{O} \cdot 0.28 \mathrm{CH}_{2} \mathrm{Cl}_{2}$ (382.46), calc: $\% \mathrm{C}$ $=74.73, \% \mathrm{H}=5.83, \% \mathrm{~N}=14.36, \% \mathrm{O}=3.94$, obs: $\% \mathrm{C}=74.68, \% \mathrm{H}=5.83, \% \mathrm{~N}=14.38$.

1-[2-(4-Benzyloxyphenyl)-6-[1,8]naphthyridin-2-yl-pyrimidin-4-yl]-ethyl ketone $\left.\mathbf{( 4}_{\mathrm{OBz}}\right)$. same protocol as for the linear butyl chain. ${ }^{1} \mathrm{H}$ NMR $\left(\mathrm{CDCl}_{3}\right): 9.20\left(\mathrm{dd},{ }^{3} \mathrm{~J}=4.2 \mathrm{~Hz},{ }^{4} \mathrm{~J}=1.9 \mathrm{~Hz}\right.$, $1 \mathrm{H}), 9.10(\mathrm{~s}, 1 \mathrm{H}), 8.88\left(\mathrm{~d},{ }^{3} J=8.4 \mathrm{~Hz}, 1 \mathrm{H}\right), 8.61\left(\mathrm{~d},{ }^{3} J=8.8 \mathrm{~Hz}, 2 \mathrm{H}\right), 8.38\left(\mathrm{~d},{ }^{3} J=8.5 \mathrm{~Hz}, 1 \mathrm{H}\right)$, $8.27\left(\mathrm{dd},{ }^{3} J=8.2 \mathrm{~Hz},{ }^{4} J=1.9 \mathrm{~Hz}, 1 \mathrm{H}\right), 7.55\left(\mathrm{dd},{ }^{3} J=8.2,{ }^{4} J=4.2 \mathrm{~Hz}, 1 \mathrm{H}\right), 7.2-7.5(\mathrm{~m}, 5 \mathrm{H}), 7.13$ $\left(\mathrm{d},{ }^{3} J=8.9 \mathrm{~Hz}, 2 \mathrm{H}\right), 5.18$ (s, 2H), 2.83 (s, 3H). ${ }^{13} \mathrm{C} \mathrm{NMR}\left(\mathrm{CDCl}_{3}\right): 199.4,164.4,164.3,161.4$, $160.7,157.2$, 154.3, 138.3, 136.9, 136.5, 130.1, 128.6, 128.1, 127.5, 123.8, 122.9, 120.0, 114.9, 111.7, 70.1, 25.8. $\mathrm{R}_{\mathrm{f}}\left(\mathrm{SiO}_{2}, \mathrm{CH}_{2} \mathrm{Cl}_{2} /\right.$ EtOAc 2.0:0.9) $=0.21 . \mathrm{mp}=211^{\circ} \mathrm{C} . \mathrm{EI}: 432.3\left(\mathrm{M}^{\circ}\right)$. EA: $\mathrm{C}_{27} \mathrm{H}_{20} \mathrm{~N}_{4} \mathrm{O}_{2} \cdot 0.036 \mathrm{CH}_{2} \mathrm{Cl}_{2}$, calc: $\% \mathrm{C}=74.56, \% \mathrm{H}=4.65, \% \mathrm{~N}=12.86, \% \mathrm{O}=7.35$, obs: $\% \mathrm{C}=$ $74.56, \% \mathrm{H}=4.51, \% \mathrm{~N}=12.91$.

7-[2-(4-n-Butylphenyl)-6-(1-ethoxyvinyl)-pyrimidin-4-yl]-pyrido-[2,3-d]-pyrimidine (6' $\left.{ }_{n B u}\right)$ :

Same protocol as for the $\mathrm{C}_{12}$ chain $\left(\mathrm{mp}=173^{\circ} \mathrm{C}\right)$; the crude product is hydrolyzed (see article text) without further purification.

$\mathbf{1}_{\text {tertBu}}$ : Same protocol as for $\mathbf{1}_{\mathbf{O B z}}$ using pyridine as a solvent. The desired product was isolated as an off-white solid by centrifugation and washing with acetone $(70 \%) .{ }^{1} \mathrm{H} \mathrm{NMR}\left(\mathrm{CDCl}_{3}\right): 9.98(\mathrm{~s}$, $1 \mathrm{H}), 9.32(\mathrm{~s}, 2 \mathrm{H}), 8.93\left(\mathrm{~d},{ }^{3} J=8.5 \mathrm{~Hz}, 2 \mathrm{H}\right), 8.81\left(\mathrm{~d},{ }^{3} J=8.3 \mathrm{~Hz}, 2 \mathrm{H}\right), 8.77\left(\mathrm{~d},{ }^{3} J=8.5 \mathrm{~Hz}, 2 \mathrm{H}\right)$, 8.5-8.6 (m, 6H), 8.35-8.45 (m, 6H), $8.21\left(\mathrm{~d},{ }^{3} J=8.5 \mathrm{~Hz}, 2 \mathrm{H}\right), 7.78\left(\mathrm{~d},{ }^{3} \mathrm{~J}=8.5 \mathrm{~Hz},{ }^{4} \mathrm{~J}=1.8 \mathrm{~Hz}\right.$, 2H), $7.67\left(\mathrm{~d},{ }^{3} J=8.4 \mathrm{~Hz}, 2 \mathrm{H}\right), 7.40\left(\mathrm{~d},{ }^{3} J=8.4 \mathrm{~Hz}, 4 \mathrm{H}\right), 6.85\left(\mathrm{dd},{ }^{3} J=8 \mathrm{~Hz},{ }^{3} J=4 \mathrm{~Hz}, 2 \mathrm{H}\right), 1.44$ $(\mathrm{s}, 9 \mathrm{H}), 1.05(\mathrm{~s}, 18 \mathrm{H}) \cdot{ }^{13} \mathrm{C} \mathrm{NMR}\left(\mathrm{CDCl}_{3}\right)$ : not soluble enough. mp $>260^{\circ} \mathrm{C}$. HR-FAB + $\left(\mathrm{C}_{83} \mathrm{H}_{54} \mathrm{~N}_{14} \mathrm{O}_{3}\right)$ : obs: $1145.5157\left(\mathrm{MH}^{+}\right)$, calc: 1145.5204 .

$\mathbf{1}_{\mathbf{n B u}}$ : Same protocol as for $\mathbf{1}_{\mathbf{O B z}}$ with an additional column purification on basic alumina $\left(\mathrm{CH}_{2} \mathrm{Cl}_{2} / \mathrm{CH}_{3} \mathrm{OH}\right.$ 2.0:0.1). ${ }^{1} \mathrm{H} \mathrm{NMR}\left(\mathrm{CDCl}_{3}\right)$ : 9.85 (br s, $\left.1 \mathrm{H}\right), 9.25$ (br s, $\left.2 \mathrm{H}\right)$, the other signals are broad (c.f. text). ${ }^{13} \mathrm{C} \mathrm{NMR}\left(\mathrm{CDCl}_{3}\right)$ : not soluble enough; $\mathrm{mp}=250^{\circ} \mathrm{C}$ (dec.). $\mathrm{HR} \mathrm{FAB}+$ $\left(\mathrm{C}_{83} \mathrm{H}_{54} \mathrm{~N}_{14} \mathrm{O}_{3}\right)$ : obs: $1145.5159\left(\mathrm{MH}^{+}\right)$, calc: 1145.5204 . 
C3N2: See general protocol for $\mathbf{C m N n}$ in the text. ${ }^{1} \mathrm{H}$ NMR $\left(\mathrm{CDCl}_{3}\right)$ : 7.6-7.8 $(\mathrm{m}, 8 \mathrm{H}), 7.4-7.5$ (m, 6H), 3.95 (s, 4H), 2.77 (t, ${ }^{3} J=6.7 \mathrm{~Hz}, 4 \mathrm{H}$ ), 1.78 (quint., ${ }^{3} J=6.7 \mathrm{~Hz}, 2 \mathrm{H}$ ), 1.68 (br s). ${ }^{13} \mathrm{C}$ NMR $\left(\mathrm{CDCl}_{3}\right)$ : 138.3, 133.8, 133.0, 128.4, 128.1, 128.0, 126.9, 126.8, 126.3, 125.8, 54.6, 48.4, 30.6. $\mathrm{R}_{\mathrm{f}}\left(\mathrm{Al}_{2} \mathrm{O}_{3}, \mathrm{CH}_{2} \mathrm{Cl}_{2} / \mathrm{CH}_{3} \mathrm{OH}\right.$ 4.5:0.1) $=0.17 . \mathrm{mp}=67^{\circ} \mathrm{C}$ (dec.; white solid). $\mathrm{FAB}+: 355.3$ $\left(\mathrm{MH}^{+}\right) . \mathrm{EA}: \mathrm{C}_{25} \mathrm{H}_{26} \mathrm{~N}_{2} \cdot 0.135 \mathrm{CH}_{2} \mathrm{Cl}_{2}$, calc: $\% \mathrm{C}=81.46, \% \mathrm{H}=7.11, \% \mathrm{~N}=7.56$, obs: $\% \mathrm{C}=$ $81.49, \% \mathrm{H}=7.00, \% \mathrm{~N}=7.65$.

C22N3: See general protocol for $\mathbf{C m N n}$ in the text. ${ }^{1} \mathrm{H}$ NMR $\left(\mathrm{CDCl}_{3}\right)$ : 7.7-7.9 $(\mathrm{m}, 8 \mathrm{H})$, 7.4-7.5 (m, 6H), 3.96 (s, 4H), 2.7-2.9 (m, 8H). ${ }^{13} \mathrm{C}$ NMR $\left(\mathrm{CDCl}_{3}\right)$ : 138.3, 133.8, 133.0, 128.4, 128.1, 128.0, 126.9, 126.8, 126.3, 125.9, 54.4, 49.6, 49.2. $\mathrm{R}_{\mathrm{f}}\left(\mathrm{Al}_{2} \mathrm{O}_{3}, \mathrm{CH}_{2} \mathrm{Cl}_{2} / \mathrm{CH}_{3} \mathrm{OH} 2.0: 0.1\right)=0.41$. $\mathrm{mp}=245^{\circ} \mathrm{C}$ (dec. Picrate, CAUTION). FAB + (picrate): $384.2\left(\mathrm{MH}^{+}\right)$. EA (picrate): $\mathrm{C}_{44} \mathrm{H}_{38} \mathrm{~N}_{12} \mathrm{O}_{21} \cdot 0.06 \mathrm{CH}_{2} \mathrm{Cl}_{2}$, calc: $\% \mathrm{C}=49.19, \% \mathrm{H}=3.57, \% \mathrm{~N}=15.41$, obs: $\% \mathrm{C}=49.19, \% \mathrm{H}=$ $3.65, \% \mathrm{~N}=15.62$.

C33N3: See general protocol for $\mathbf{C m N n}$ in the text. ${ }^{1} \mathrm{H}$ NMR $\left(\mathrm{CDCl}_{3}\right)$ : 7.7-7.9 (m, 8H), 7.4-7.5 (m, 6H), $3.91(\mathrm{~s}, 4 \mathrm{H}), 2.72\left(\mathrm{t},{ }^{3} J=7.0 \mathrm{~Hz}, 4 \mathrm{H}\right), 2.68$ (t, $\left.{ }^{3} J=6.9 \mathrm{~Hz}, 4 \mathrm{H}\right), 1.71$ (quint., ${ }^{3} J=6.9$ $\mathrm{Hz}, 4 \mathrm{H}) .{ }^{13} \mathrm{C} \mathrm{NMR}\left(\mathrm{CDCl}_{3}\right): 138.3,133.8,133.0,128.4,128.0,126.9,126.8,126.4,126.0,125.8$, 54.5, 48.9, 48.3, 30.4. $\mathrm{R}_{\mathrm{f}}\left(\mathrm{Al}_{2} \mathrm{O}_{3}, \mathrm{CH}_{2} \mathrm{Cl}_{2} / \mathrm{CH}_{3} \mathrm{OH}\right.$ 2.0: 0.1) $=0.17 . \mathrm{mp}=260^{\circ} \mathrm{C}$ (dec. Picrate, CAUTION). FAB+ (picrate): $412.4\left(\mathrm{MH}^{+}\right)$. EA (picrate): $\mathrm{C}_{46} \mathrm{H}_{42} \mathrm{~N}_{12} \mathrm{O}_{21} \cdot 0.425 \mathrm{CHCl}_{3}$, calc: $\% \mathrm{C}=$ $48.50, \% \mathrm{H}=3.72, \% \mathrm{~N}=14.54$, obs: $\% \mathrm{C}=48.49, \% \mathrm{H}=3.66, \% \mathrm{~N}=14.80$.

C343N3: See general protocol for $\mathbf{C m N n}$ in the text, starting from $300 \mathrm{mg}$ of commercially available spermine $(1.5 \mathrm{mmol})$. The yield was greatly diminished upon purification. ${ }^{1} \mathrm{H}$ NMR $\left(\mathrm{CDCl}_{3}\right)$ : 7.7-7.9 (m, 8H), 7.4-7.5 (m, 6H), $3.94(\mathrm{~s}, 4 \mathrm{H}), 2.5-2.8(\mathrm{~m}, 12 \mathrm{H}), 1.7-1.9(\mathrm{~m}, 8 \mathrm{H}) . \mathrm{R}_{\mathrm{f}}$ $\left(\mathrm{Al}_{2} \mathrm{O}_{3}, \mathrm{CH}_{2} \mathrm{Cl}_{2} / \mathrm{CH}_{3} \mathrm{OH}\right.$ 2:0:0:1) $=0.15$.

N-Benzylprolin-3-benzylaminopropylamide (ProAC3N2): Same protocol as for ProAN1 with an additional 20h heating. ${ }^{1} \mathrm{H}$ NMR $\left(\mathrm{CDCl}_{3}\right)$ : $7.60(\mathrm{br} \mathrm{t}, 1 \mathrm{H}), 7.2-7.4(\mathrm{~m}, 10 \mathrm{H}), 3.82\left(\mathrm{~d},{ }^{2} J=13.1\right.$ $\mathrm{Hz}, 1 \mathrm{H}$ ), 3.75 (s, 2H), 3.46 (d, ${ }^{2} J=13.1 \mathrm{~Hz}, 1 \mathrm{H}$ ), 3.30 (quadr., ${ }^{3} J=6.5 \mathrm{~Hz}, 2 \mathrm{H}$ ), 3.18 (dd, ${ }^{3} J=$ $\left.9.9 \mathrm{~Hz},{ }^{3} J=5.0 \mathrm{~Hz}, 1 \mathrm{H}\right), 3.0(\mathrm{~m}, 1 \mathrm{H}), 2.63\left(\mathrm{t},{ }^{3} J=6.6 \mathrm{~Hz}, 2 \mathrm{H}\right), 2.1-2.4(\mathrm{~m}, 2 \mathrm{H}), 1.5-1.9(\mathrm{~m}, 5 \mathrm{H})$. ${ }^{13} \mathrm{C} \mathrm{NMR}\left(\mathrm{CDCl}_{3}\right)$ : 174.9, 140.7, 129.0, 128.7, 128.6, 128.3, 127.5, 127.2, 67.8, 60.2, 54.3, 47.2, 37.4, 30.2, 24.4.

N-Benzylproline-3-(3-benzylaminopropylamino)-propylamide (ProAC33N3): Same protocol as for ProAN1 with a total heating time of $24 \mathrm{~h} .{ }^{1} \mathrm{H}$ NMR $\left(\mathrm{CDCl}_{3}\right)$ : $7.6(\mathrm{br} \mathrm{t}, 1 \mathrm{H}), 7.1-7.4(\mathrm{~m}$, $10 \mathrm{H}), 3.86\left(\mathrm{~d},{ }^{2} J=13.1 \mathrm{~Hz}, 1 \mathrm{H}\right), 3.78(\mathrm{~s}, 2 \mathrm{H}), 3.50\left(\mathrm{~d},{ }^{2} J=13.1 \mathrm{~Hz}, 1 \mathrm{H}\right), 3.1-3.4(\mathrm{~m}, 3 \mathrm{H}), 3.0-$ 
$3.15(\mathrm{~m}, 1 \mathrm{H}), 2.5-2.8(\mathrm{~m}, 6 \mathrm{H}), 2.1-2.5(\mathrm{~m}, 2 \mathrm{H}), 1.5-2.0(\mathrm{~m}, 7 \mathrm{H}) .{ }^{13} \mathrm{C} \mathrm{NMR}\left(\mathrm{CDCl}_{3}\right)$ : 174.6, 140.5, 138.6, 128.7, 128.4, 128.3, 128.0, 127.2, 126.8, 67.4, 59.9, 54.0, 53.5, 48.5, 47.8, 47.4, 37.0, 30.8, 30.0, 29.9, 24.1. FAB +: $409.3\left(\mathrm{MH}^{+}\right), 160.1\left(\left(\mathrm{M}-\mathrm{CONHC}_{13} \mathrm{H}_{21} \mathrm{~N}_{2}\right) \mathrm{H}^{+}\right)$.

N-Benzylprolin-3-benzylaminopropylamine (ProC3N2): Same protocole as for ProN1 using $\mathrm{CH}_{2} \mathrm{Cl}_{2} /$ hexane/Et $3 \mathrm{~N}$ 4.0:1.0:0.4 to 2.0:0:0.2 as an eluent to yield the desired product as a turpid oil (1.54 g, $85 \%$ for both transamidation and reduction steps). ${ }^{1} \mathrm{H}$ NMR $\left(\mathrm{CDCl}_{3}\right)$ : 7.2-7.4 (m, $5 \mathrm{H}), 3.97\left(\mathrm{~d},{ }^{2} J=13.1 \mathrm{~Hz}, 1 \mathrm{H}\right), 3.79(\mathrm{~s}, 2 \mathrm{H}), 3.33\left(\mathrm{~d},{ }^{2} J=13.1 \mathrm{~Hz}, 1 \mathrm{H}\right), 2.85-3.0(\mathrm{~m}, 1 \mathrm{H}), 2.5-$ $2.8(\mathrm{~m}, 5 \mathrm{H}), 2.20$ (quadr., $\left.{ }^{3} \mathrm{~J}=8.2 \mathrm{~Hz}, 1 \mathrm{H}\right), 1.6-2.0(\mathrm{~m}, 7 \mathrm{H}) .{ }^{13} \mathrm{C} \mathrm{NMR}\left(\mathrm{CDCl}_{3}\right)$ : 140.8, 140.3, 128.9, 128.6, 128.4, 128.3, 127.0, 126.9, 64.1, 59.6, 54.9, 54.4, 53.5, 49.3, 48.4, 30.5, 29.4, 23.2. $\mathrm{R}_{\mathrm{f}}\left(\mathrm{SiO}_{2}, \mathrm{CH}_{2} \mathrm{Cl}_{2} /\right.$ hexane/ $\mathrm{Et}_{3} \mathrm{~N}$ 1.0:2.0:0.1) = 0.28. EA: $\mathrm{C}_{22} \mathrm{H}_{31} \mathrm{~N}_{3} \cdot 0.17 \mathrm{CH}_{2} \mathrm{Cl}_{2}$, calc: $\% \mathrm{C}=$ $75.73, \% \mathrm{H}=8.98, \% \mathrm{~N}=11.95$, obs: $\% \mathrm{C}=75.76, \% \mathrm{H}=9.10, \% \mathrm{~N}=11.30$.

N-Benzylproline-3-(3-benzylaminopropylamino)-propylamine (ProC33N3): Idem as for ProN1 using $\mathrm{CH}_{2} \mathrm{Cl}_{2} / \mathrm{CH}_{3} \mathrm{OH} / \mathrm{Et}_{3} \mathrm{~N}$ 2.0:0.3:0.2 as an eluent to yield the desired product as a turbid oil (770 mg, $36 \%$ yield for both transamidation and reduction steps). ${ }^{1} \mathrm{H}$ NMR $\left(\mathrm{CDCl}_{3}\right)$ : 7.1-7.4 (m, 5H), $3.96\left(\mathrm{~d},{ }^{2} J=13.1 \mathrm{~Hz}, 1 \mathrm{H}\right), 3.78(\mathrm{~s}, 2 \mathrm{H}), 3.31\left(\mathrm{~d},{ }^{2} J=13.1 \mathrm{~Hz}, 1 \mathrm{H}\right), 2.85-3.0(\mathrm{~m}$,

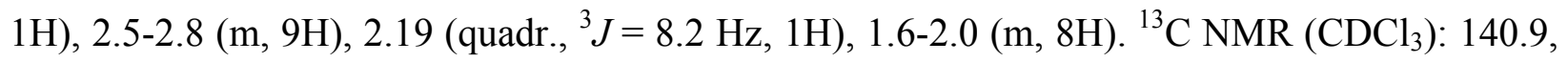
140.4, 129.0, 128.7, 128.5, 128.4, 127.2, 127.1, 64.1, 59.7, 54.9, 54.4, 53.5, 49.3, 49.0, 48.9, 48.3, 34.5, 30.6, 29.5, 23.2. $\mathrm{R}_{\mathrm{f}}\left(\mathrm{SiO}_{2}, \mathrm{CH}_{2} \mathrm{Cl}_{2} /\right.$ hexane/Et $3 \mathrm{~N}$ 1.0: 2.0:0.1) $=0.28 . \mathrm{FAB}+$ : 395.3 $\left(\mathrm{MH}^{+}\right), 160.1\left(\left(\mathrm{M}_{-} \mathrm{CH}_{2} \mathrm{NHC}_{13} \mathrm{H}_{21}\right) \mathrm{H}^{+}\right)$. EA: $\mathrm{C}_{25} \mathrm{H}_{38} \mathrm{~N}_{4} \cdot 0.54 \mathrm{CH}_{2} \mathrm{Cl}_{2}$, calc: \%C $=69.70, \% \mathrm{H}$ $=8.95, \% \mathrm{~N}=12.73$, obs: $\% \mathrm{C}=69.68, \% \mathrm{H}=8.95, \% \mathrm{~N}=12.03$.

\section{References:}

(1) Bredereck, H.; Simchen, G.; Traut; H. Chem. Ber. 1967, 100, 3664-3670.

(2) (a) Osselaere, J.P.; Dejardin, J.V.; Dejardin-Duchêne, M. Bull. Soc. Chim. Belges 1969, 78, 289-298. (b) Majewicz, T.G.; Caluwe, P. J. Org. Chem. 1974, 39, 720-721.

(3) Cuccia, L.A.; Ruiz, E.; Lehn, J.-M.; Homo, J.-C.; Schmutz, M. Chem. Eur. J. 2002, 8, 34483457.

(4) Von Schubert, H.; Zachke, H. J. Prakt. Chem. 1970, 312, 494-506.

(5) Burdeska, v.K.; Fuhrer, H; Kabas, G.; Siegrist, A.E. Helv. Chim. Acta. 1981, 64, 113-152. 


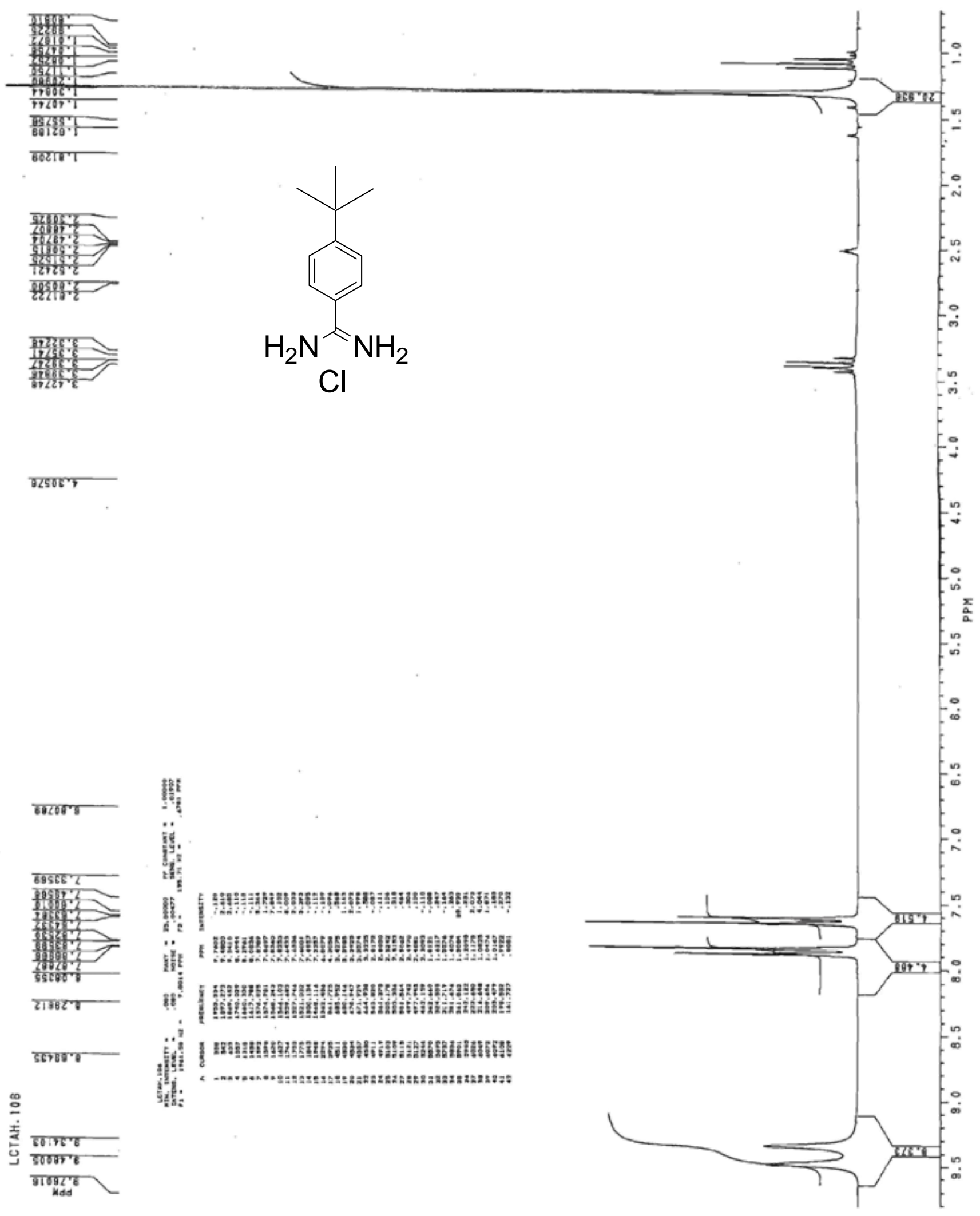




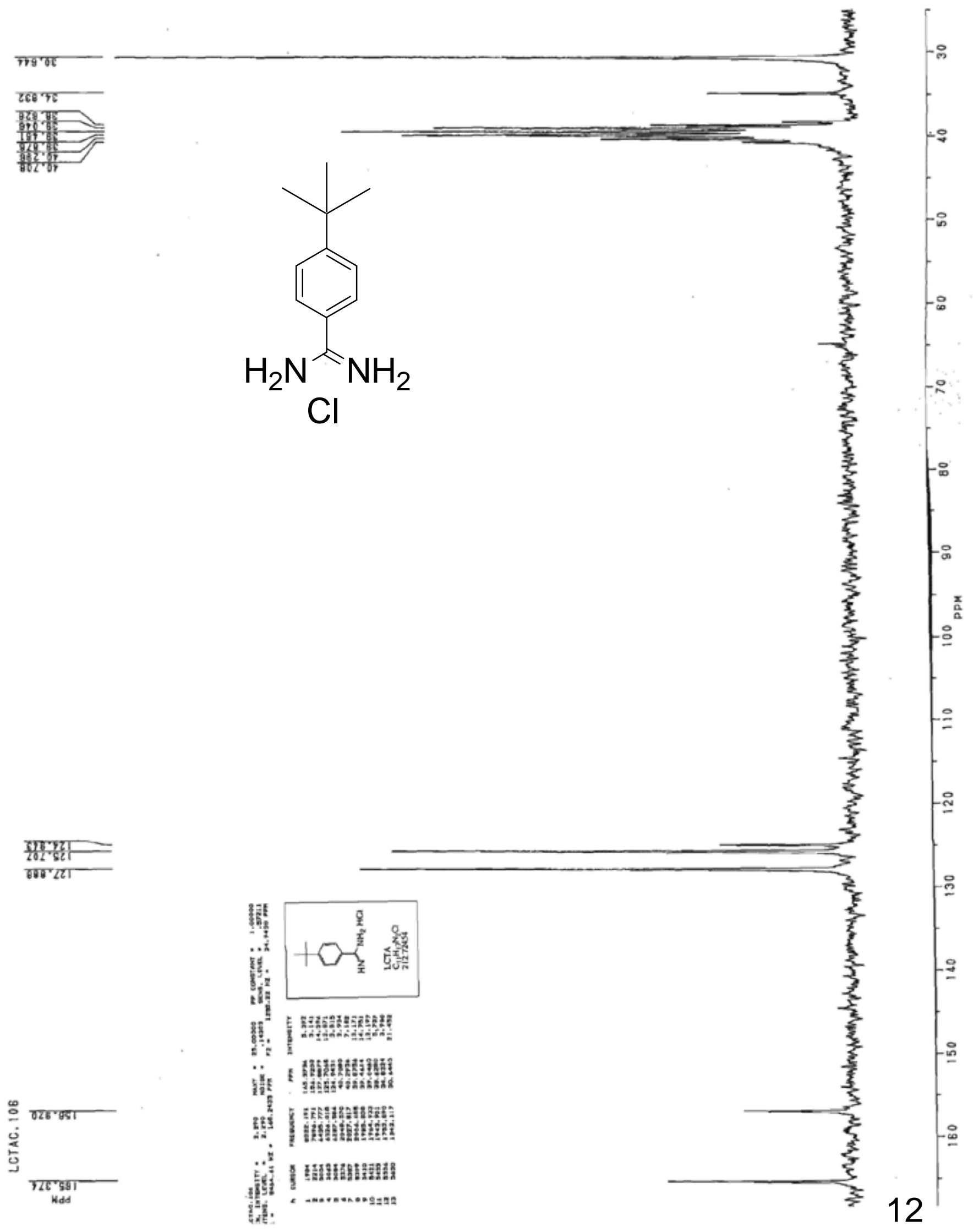




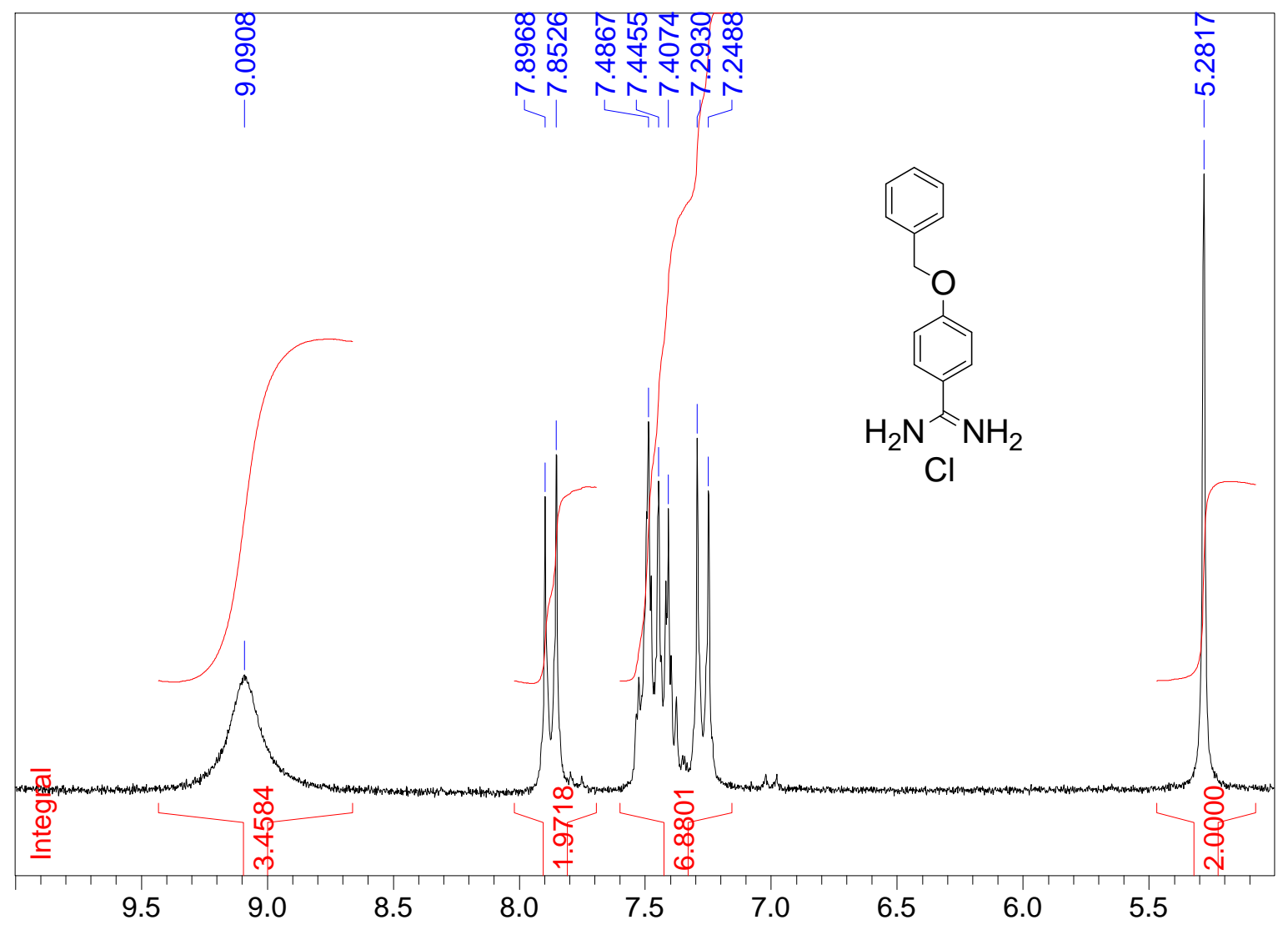

(ppm)

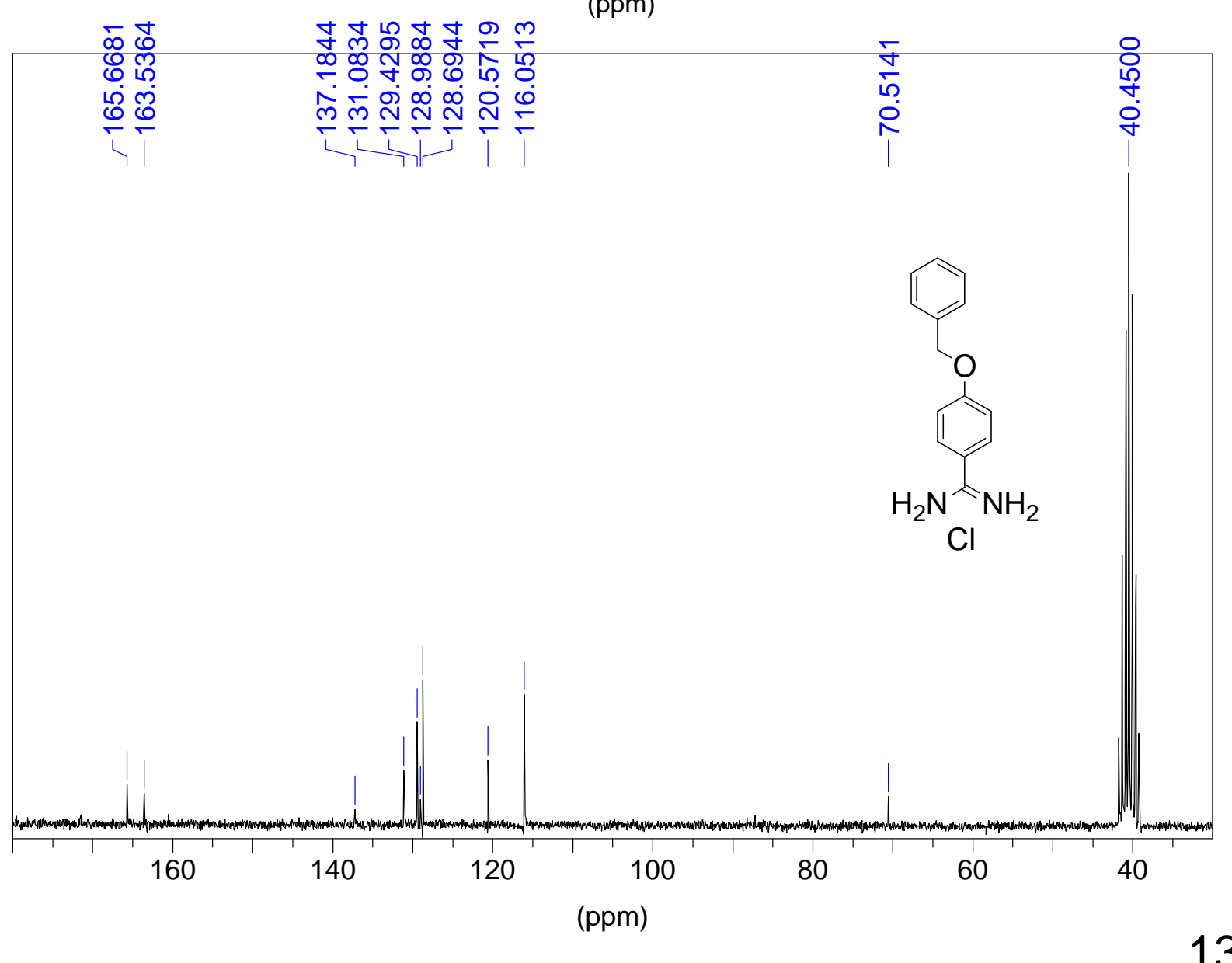




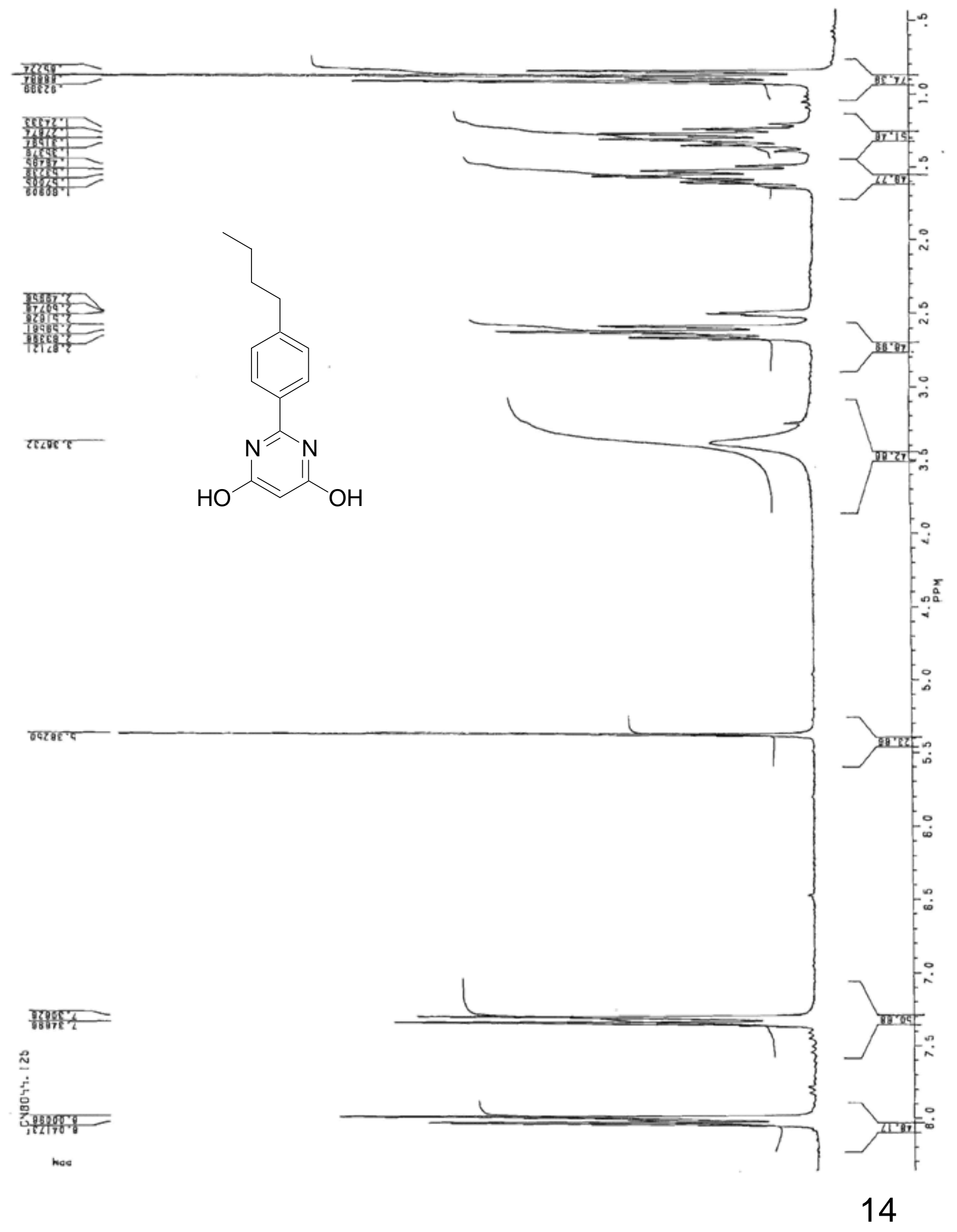


${ }^{0 \varepsilon z 9^{\circ}} \varepsilon I-$

ZSE9 $T Z-$

$t+29^{\circ} \tau \mathcal{L}-$

SSES'T -

$6988.6 \varepsilon-$<smiles>CCCCc1ccc(-c2nc(O)cc(O)n2)cc1</smiles>

\$85I 88

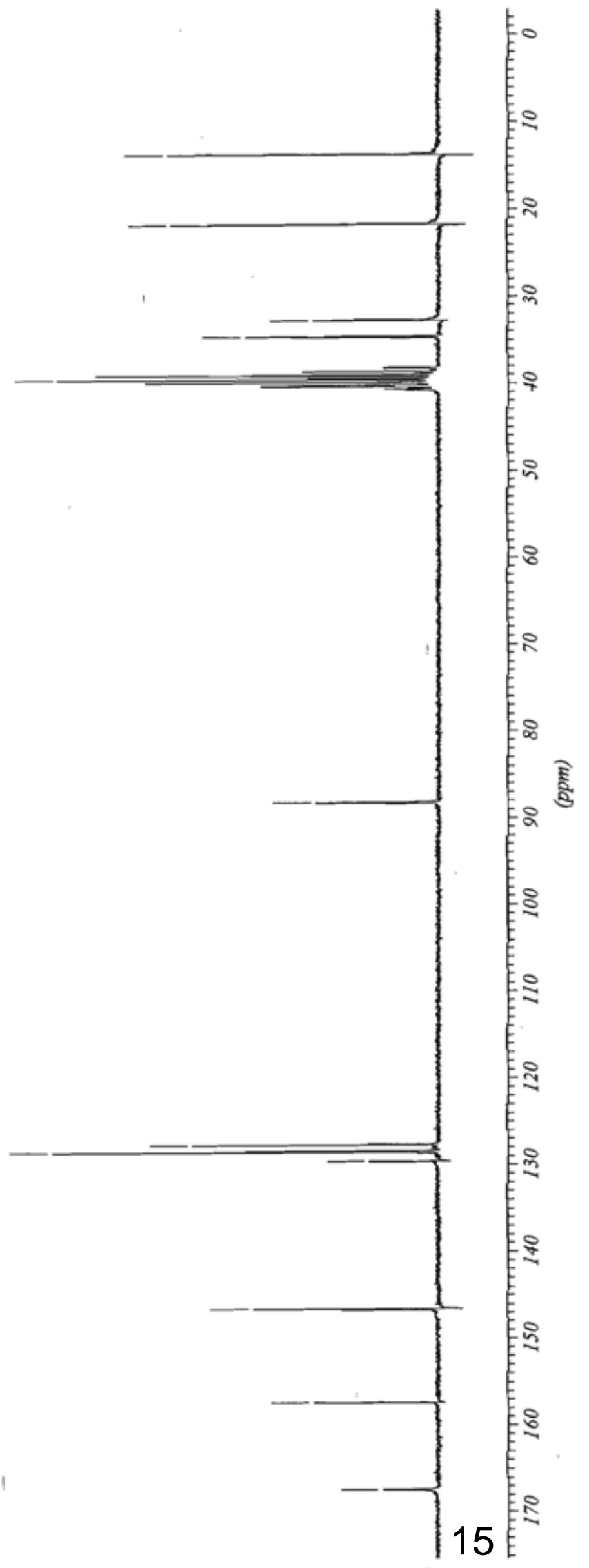




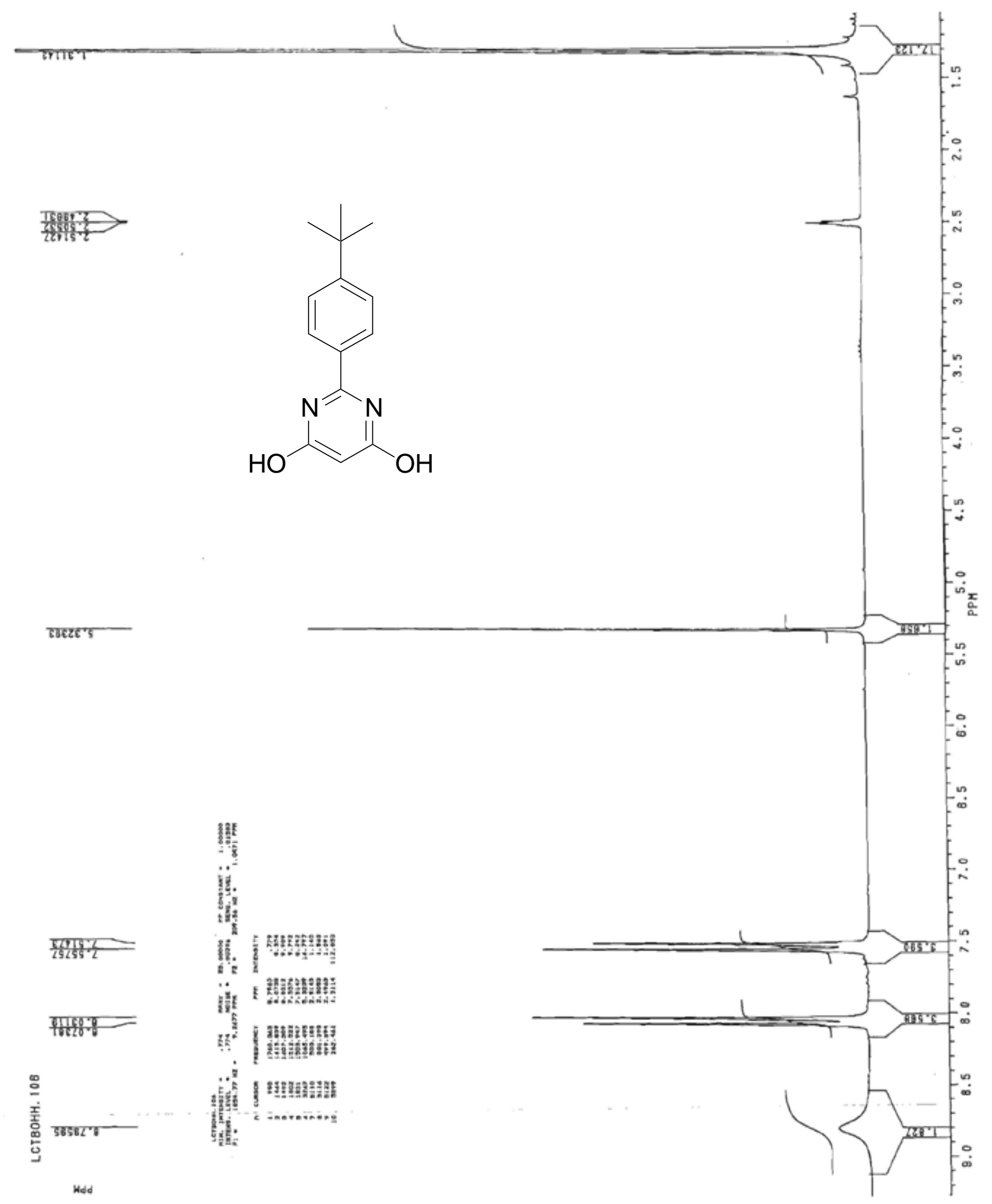

16 

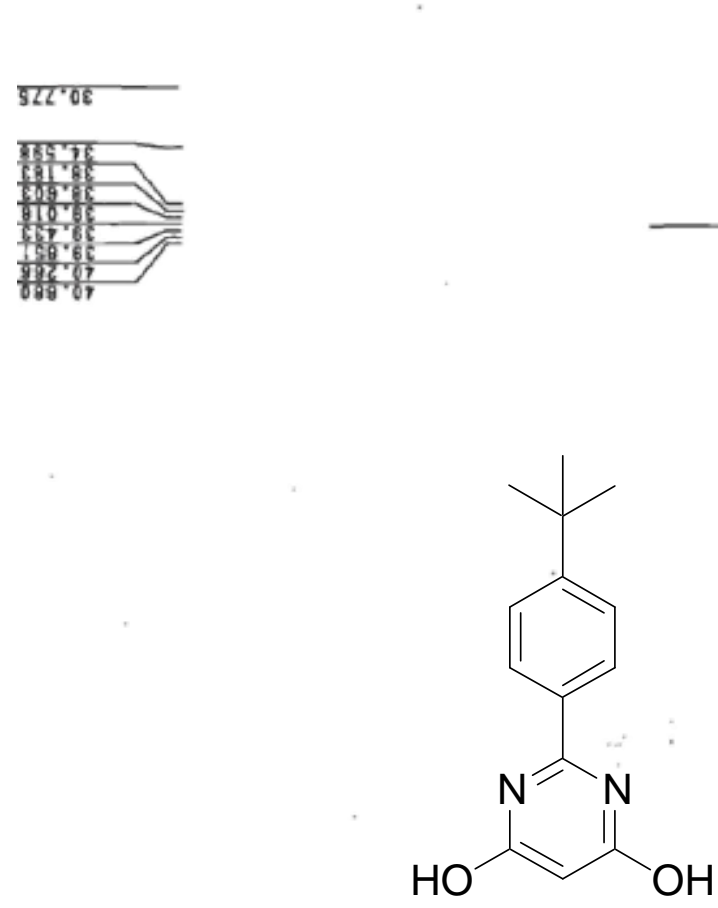

हाग 8
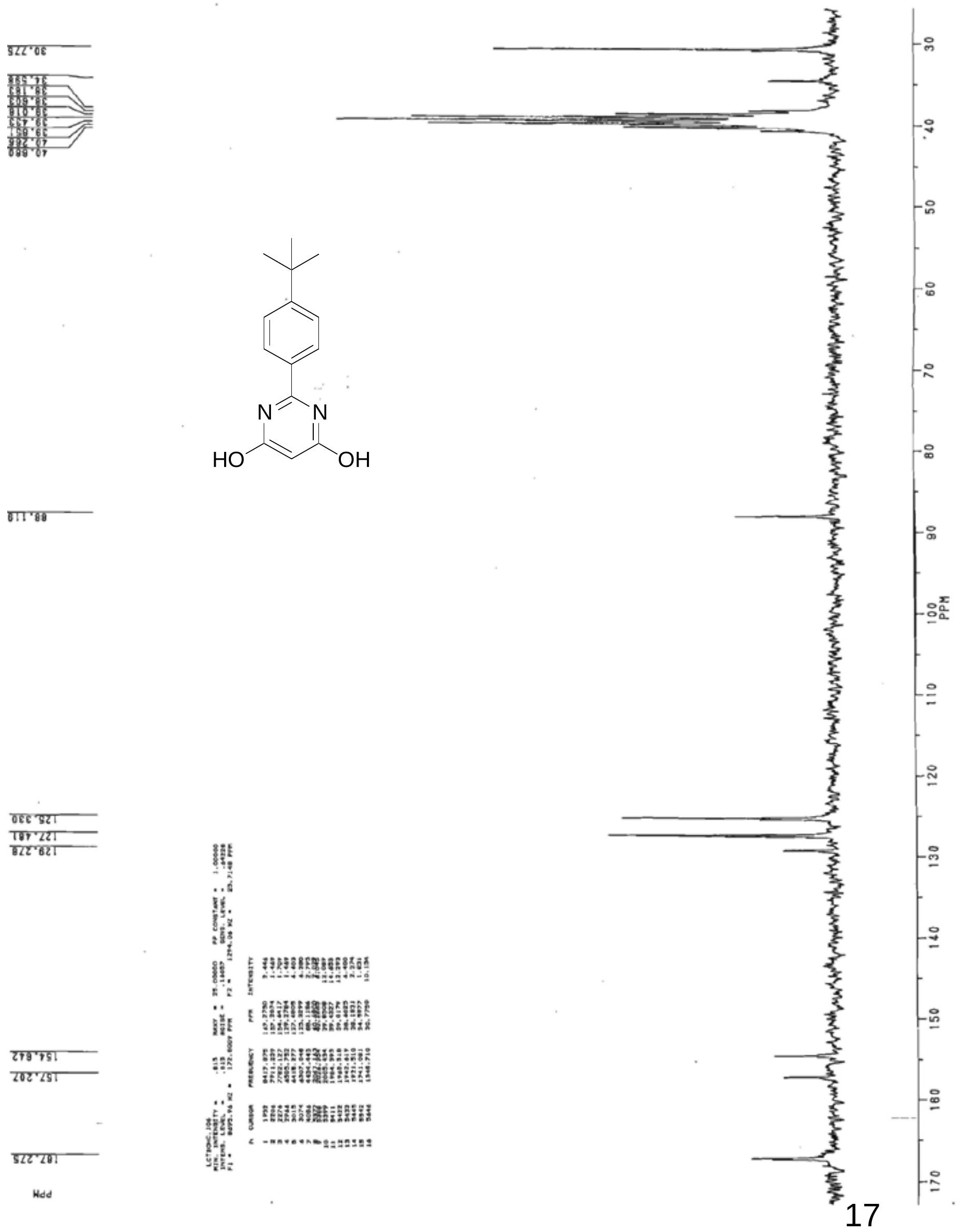


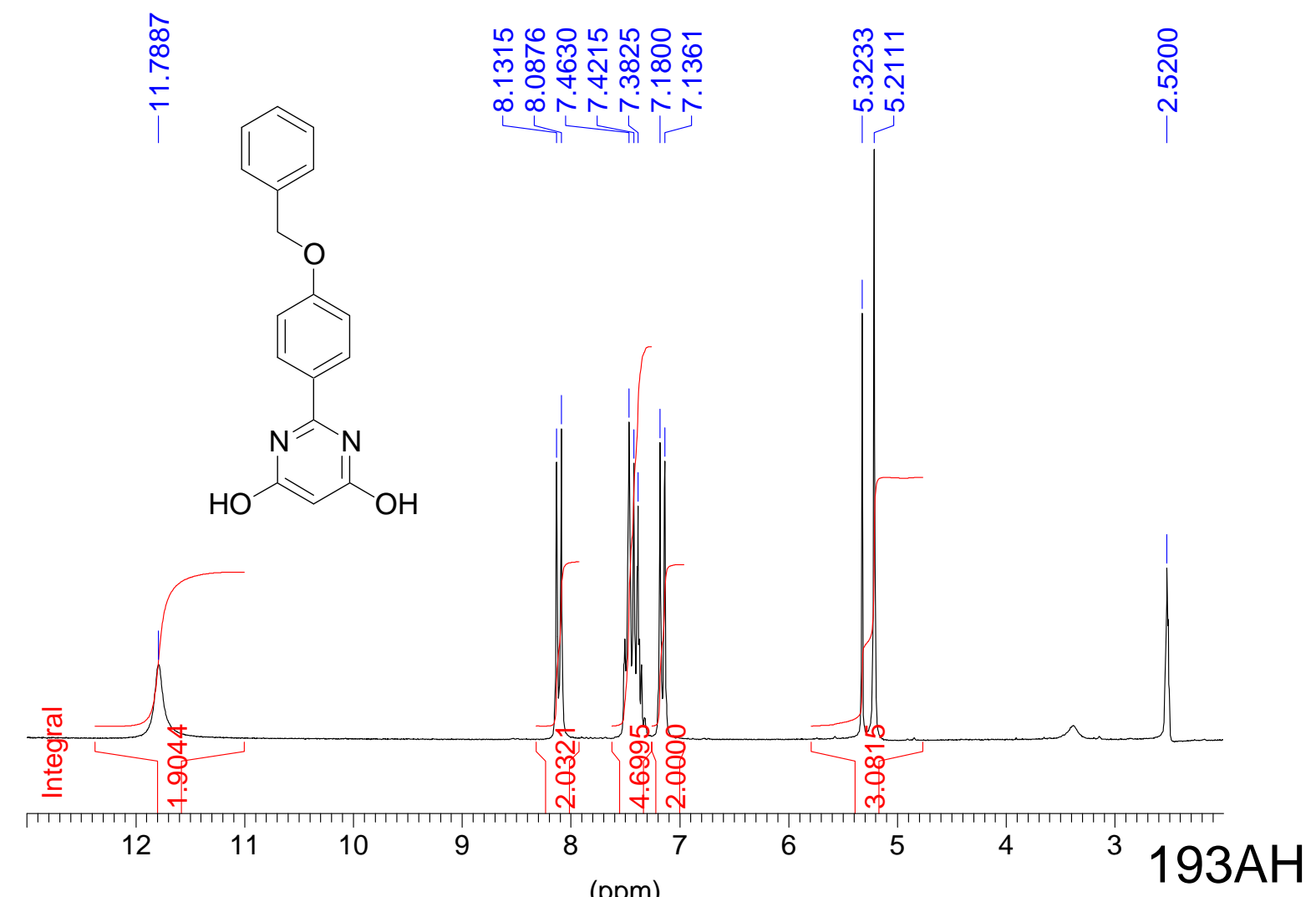

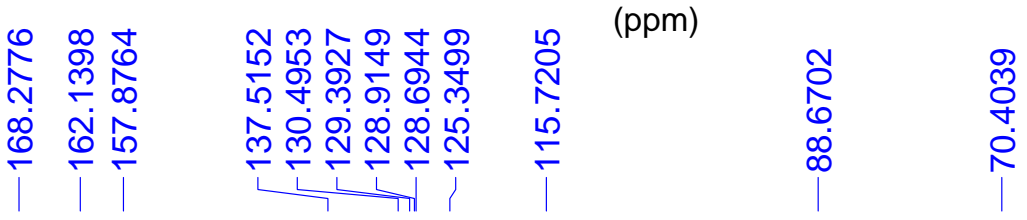

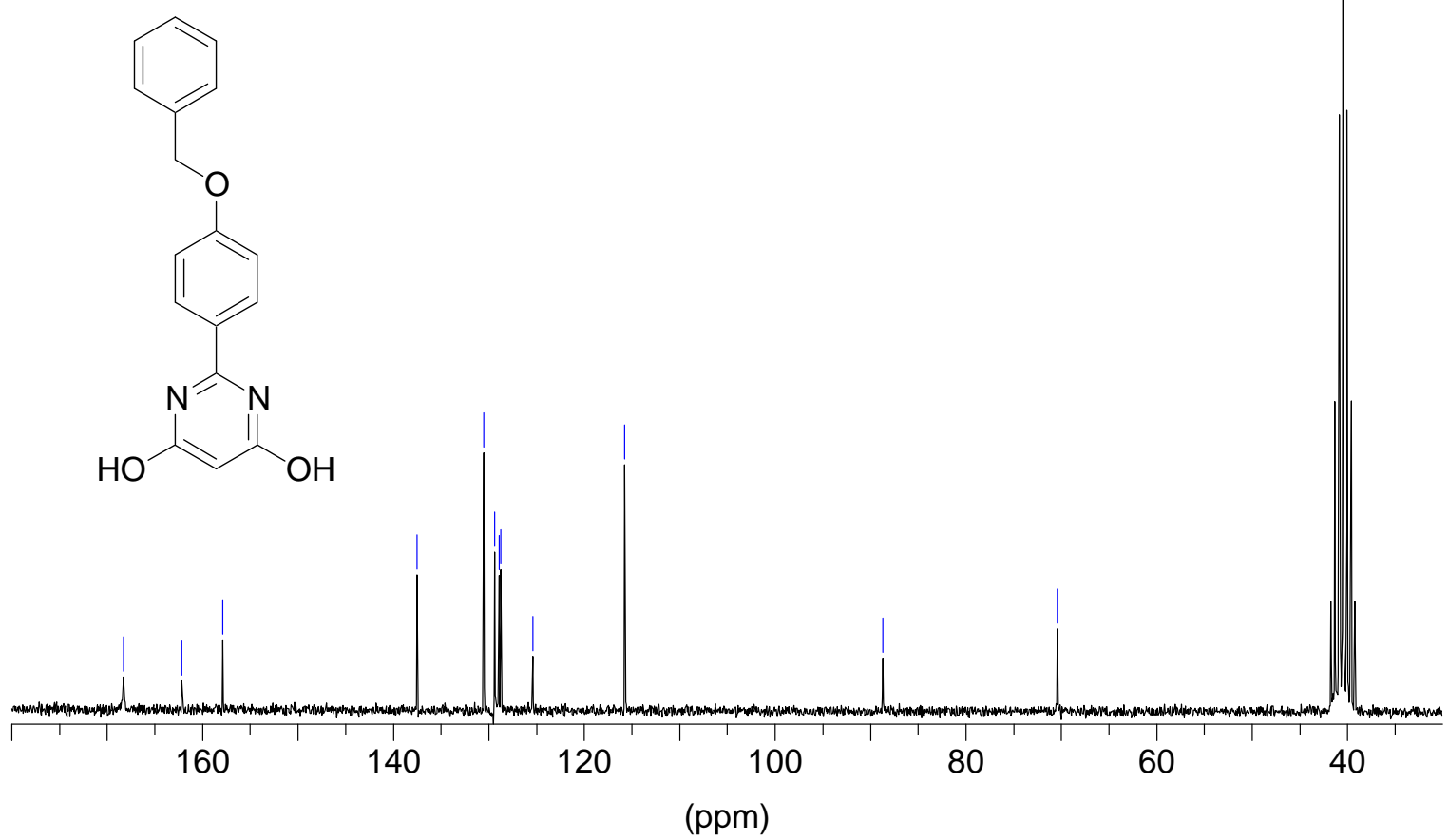


ֻ

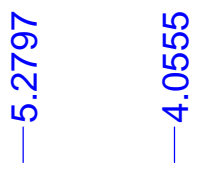

유ำ กิ ํํำ

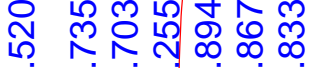

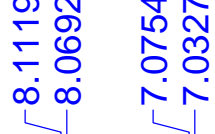

N
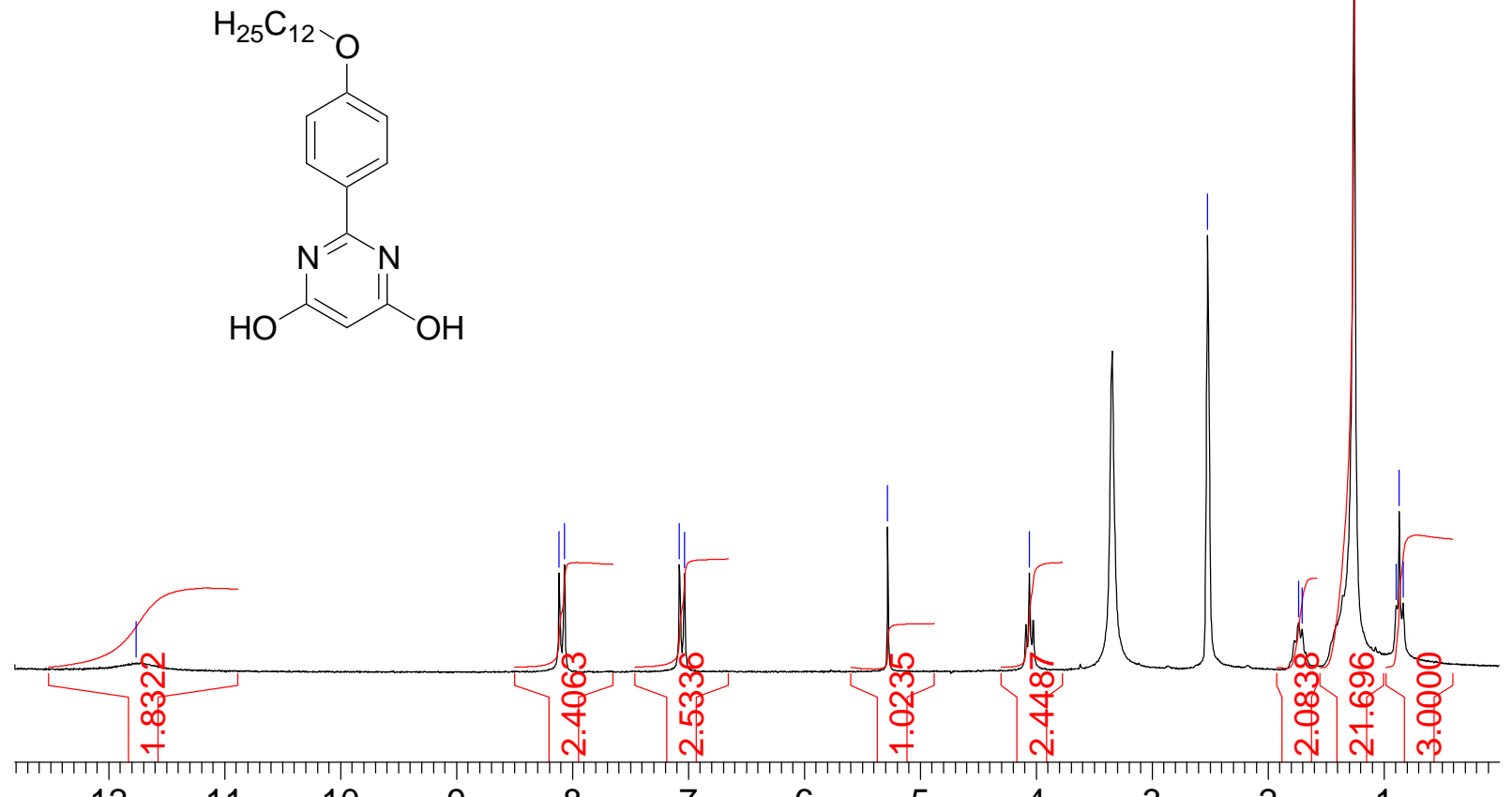

12

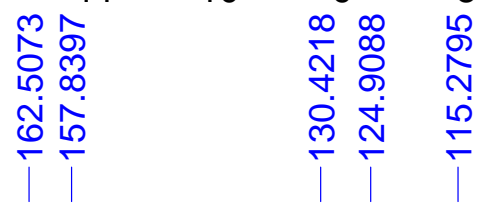

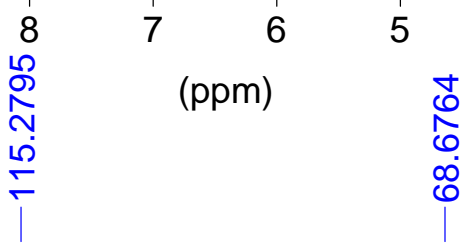<smiles>CCCCCCCCCOc1ccc(-c2nc(O)cc(O)n2)cc1</smiles>

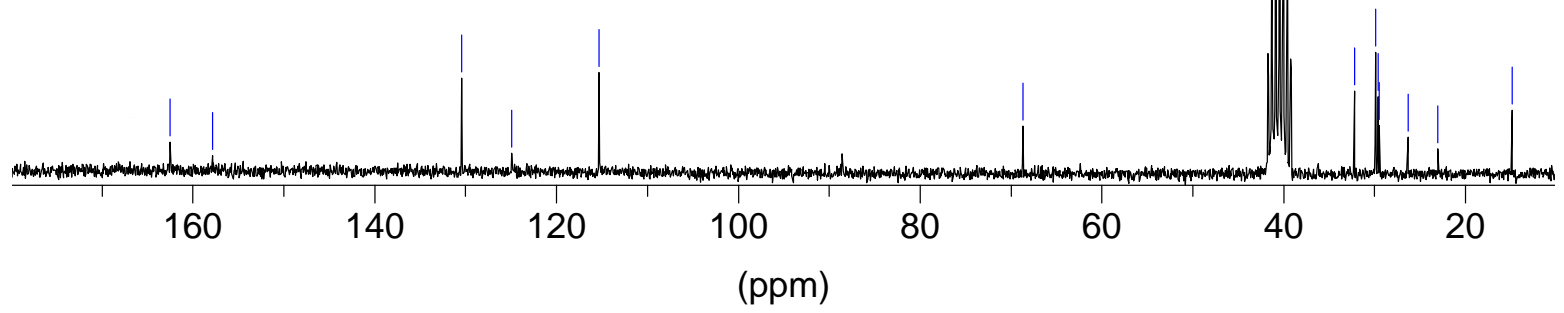



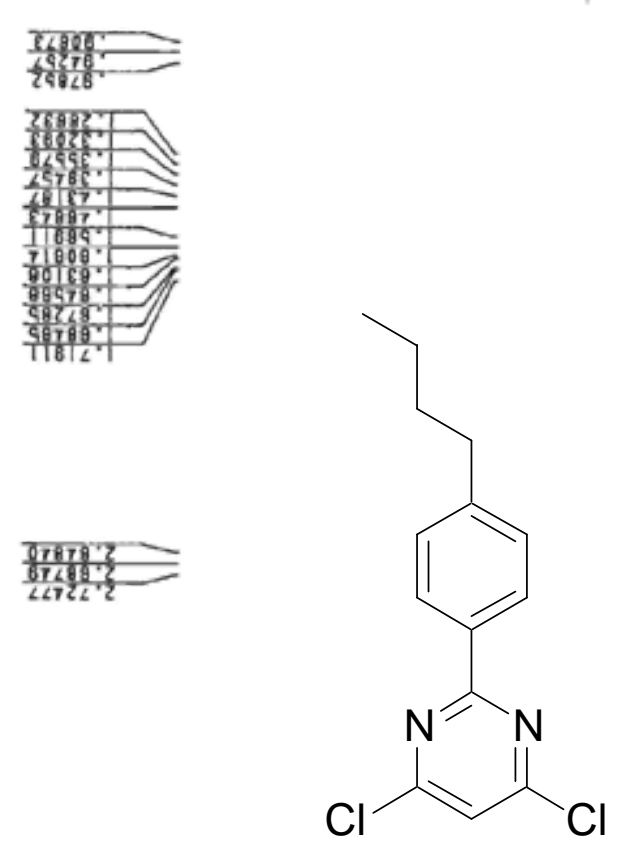

$\overline{18687^{\circ} \mathrm{T}}$

पहतहE'द

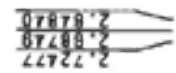

-

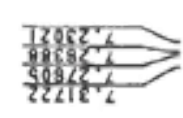


$2196^{\circ}$ ' V $\angle \angle 68^{\circ} 921^{\circ}$

$t+E^{\prime} 22 \quad 218+8 I^{-}-$
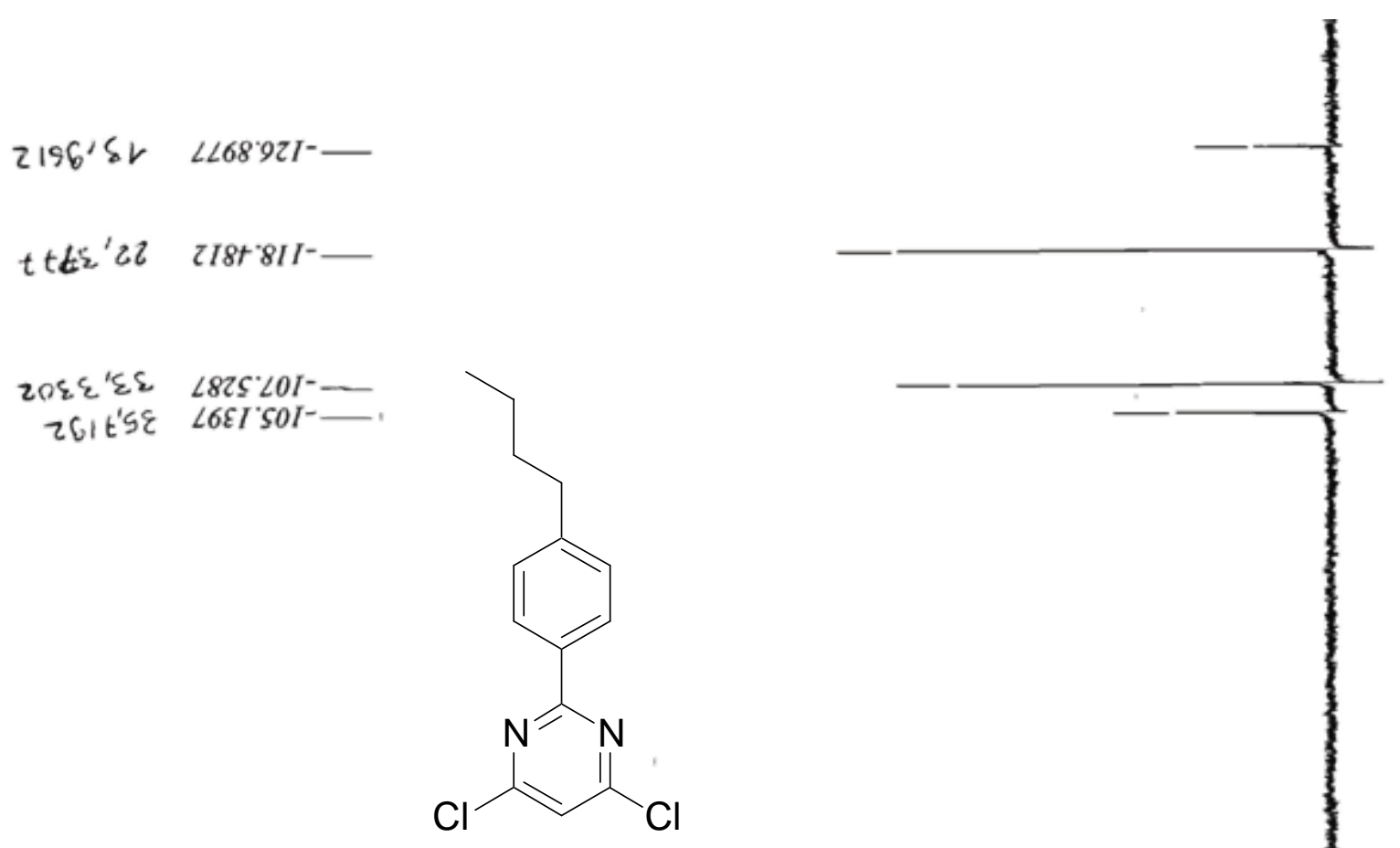

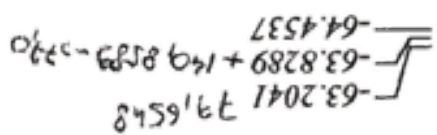

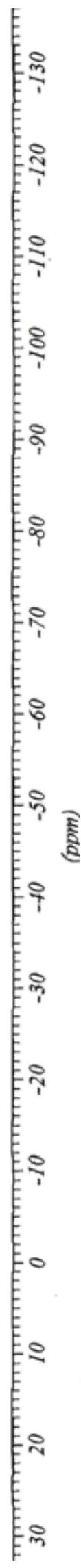

21

EG'r \& EILOIZ -

प6's9L $t \angle \angle 0 S Z-$

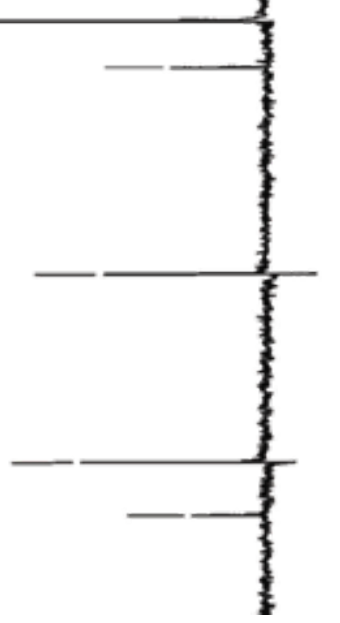




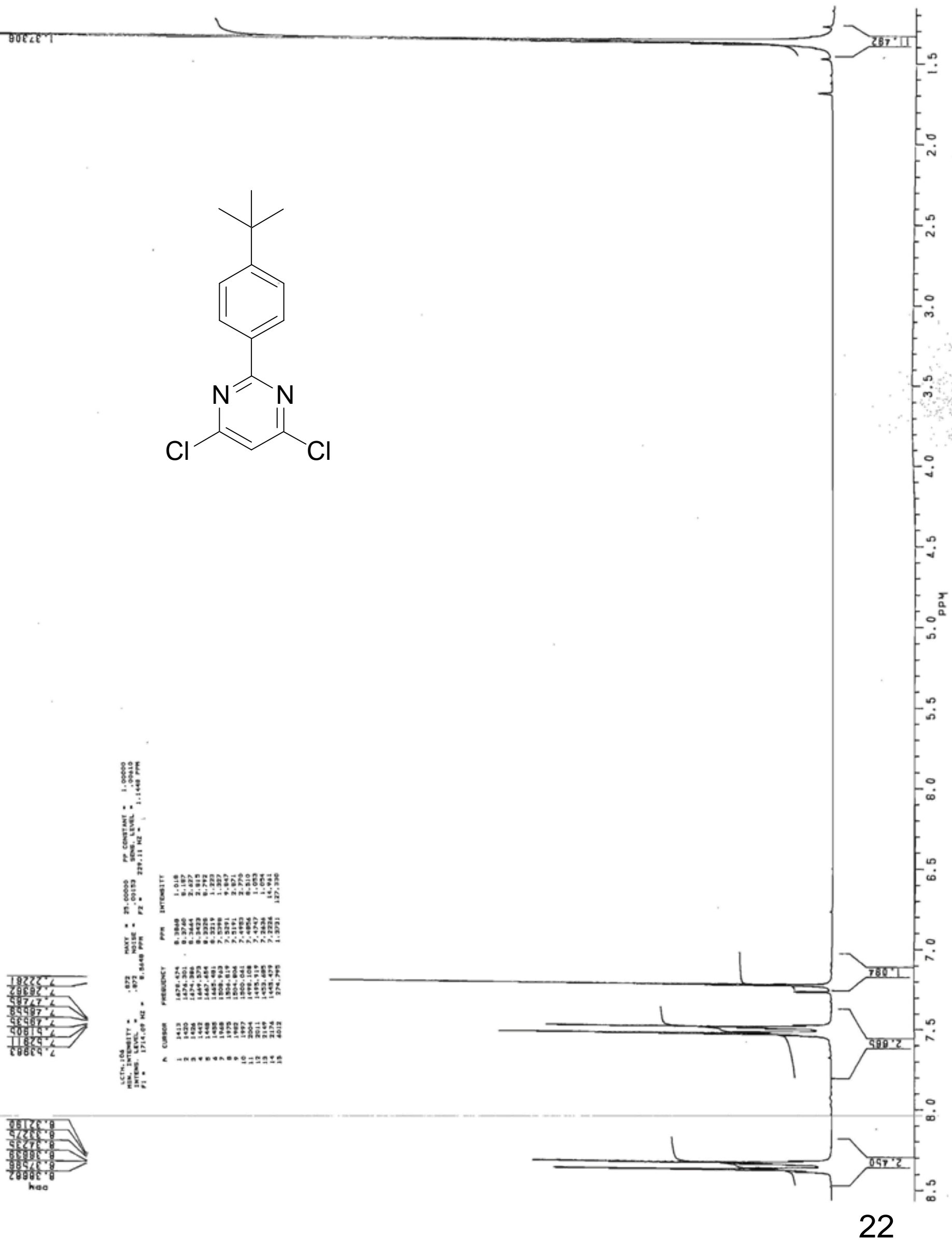



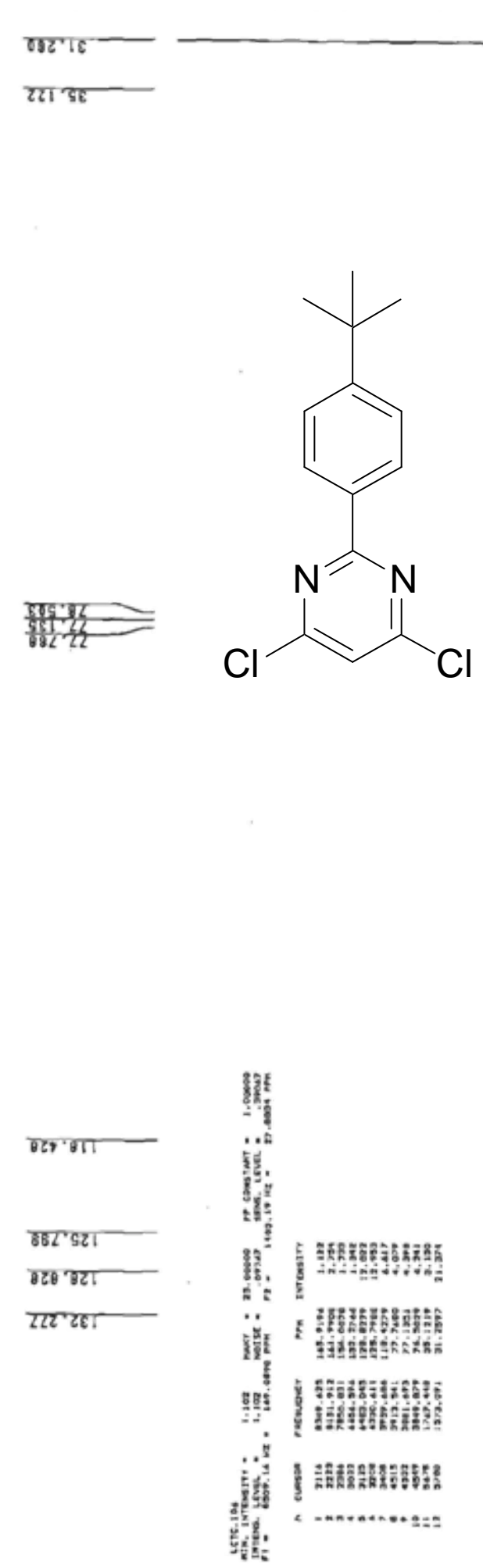

800 'gडा

TहB 191

गदहजश

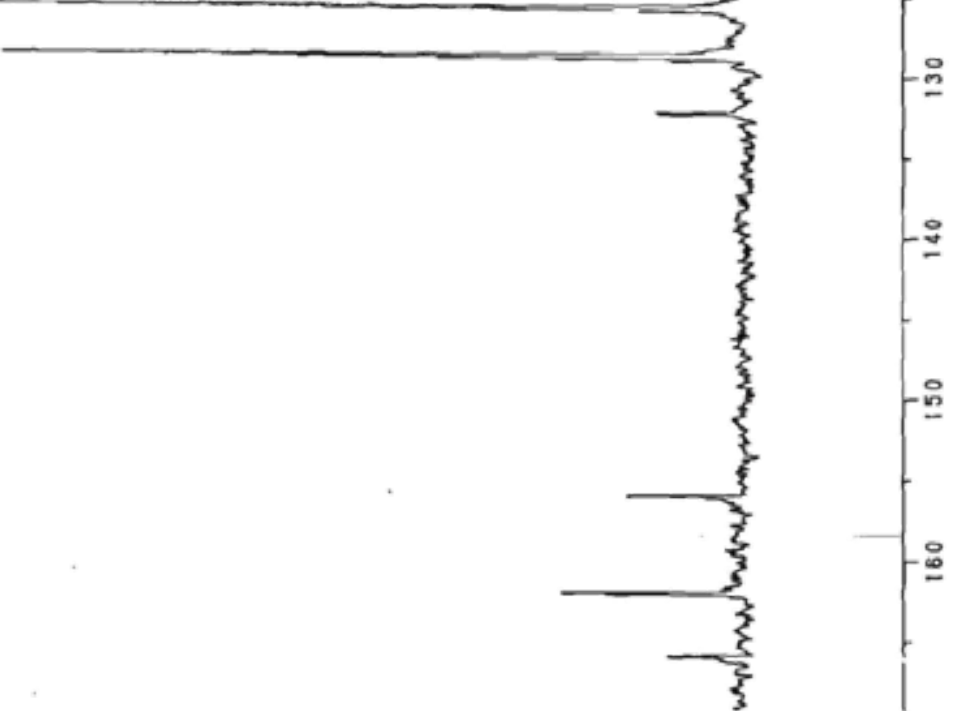

23 
चष्ताव -<smiles>Clc1cc(Cl)nc(-c2ccc(OCc3ccccc3)cc2)n1</smiles>
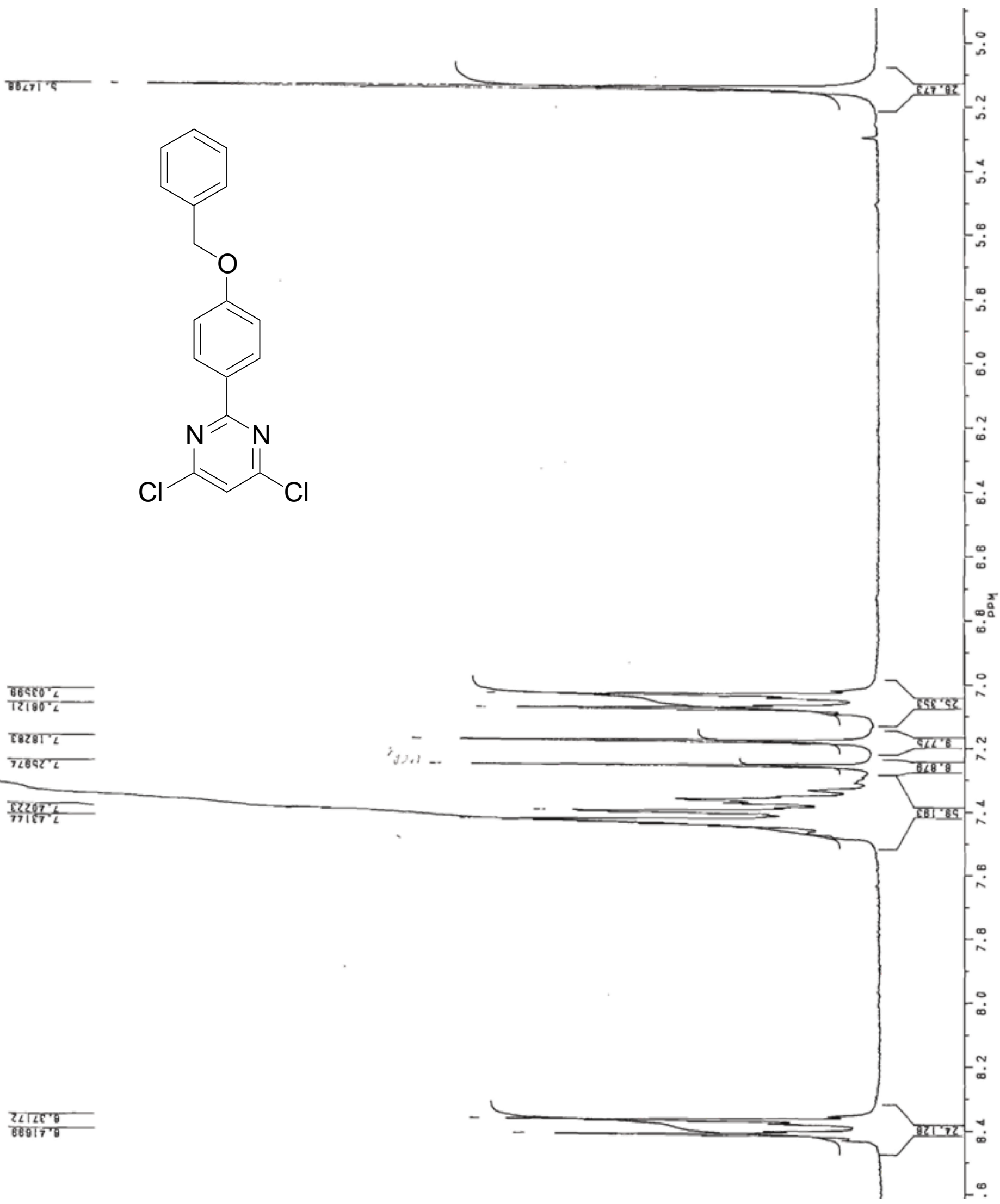

24 


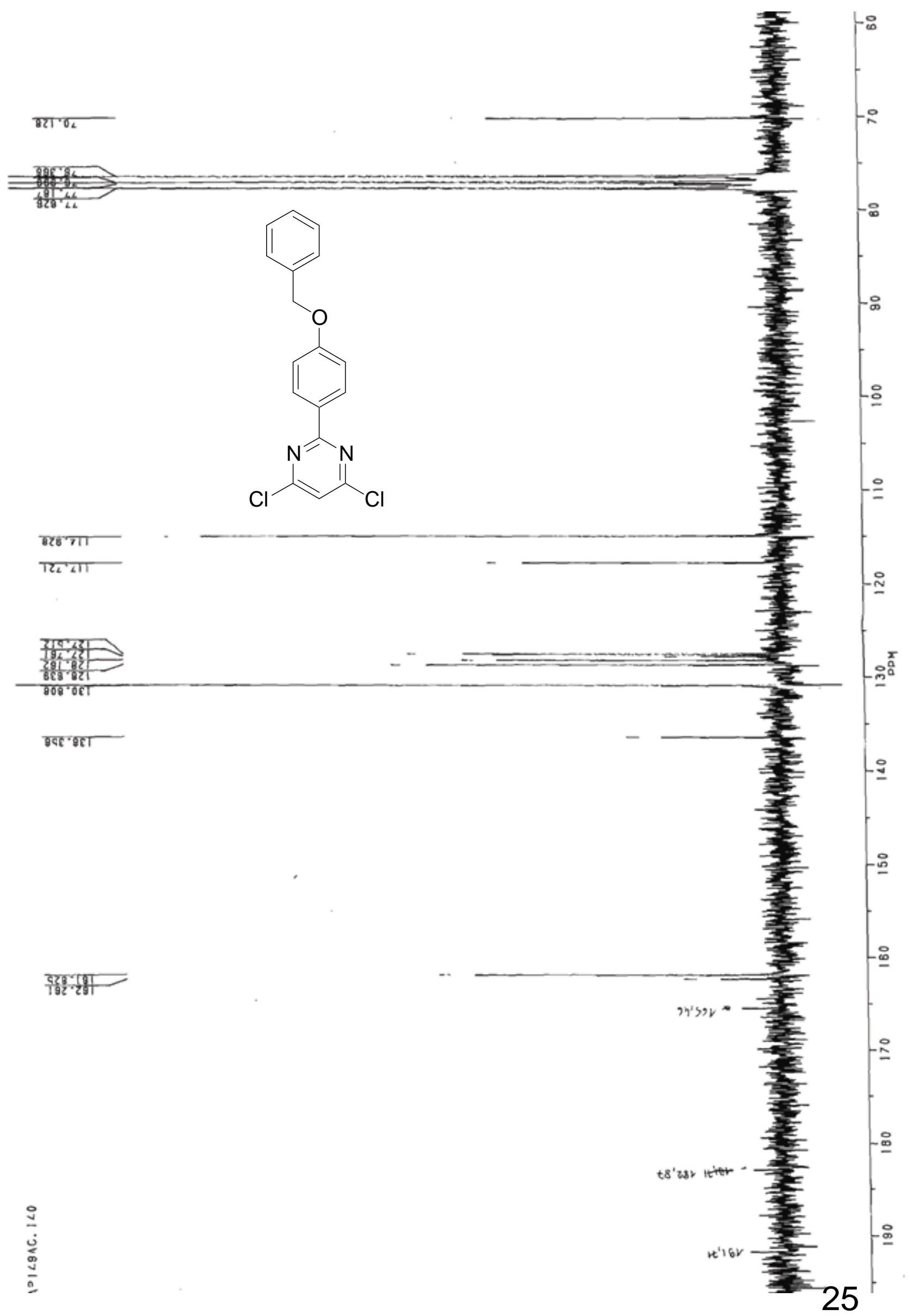




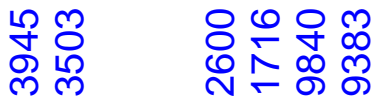

$\infty \infty \quad N$

ஸิ $\infty$

ช ป

षं

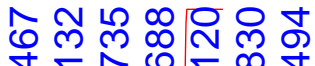

め

ت<smiles>CCCCCCCCCOc1ccc(-c2nc(Cl)cc(Cl)n2)cc1</smiles>
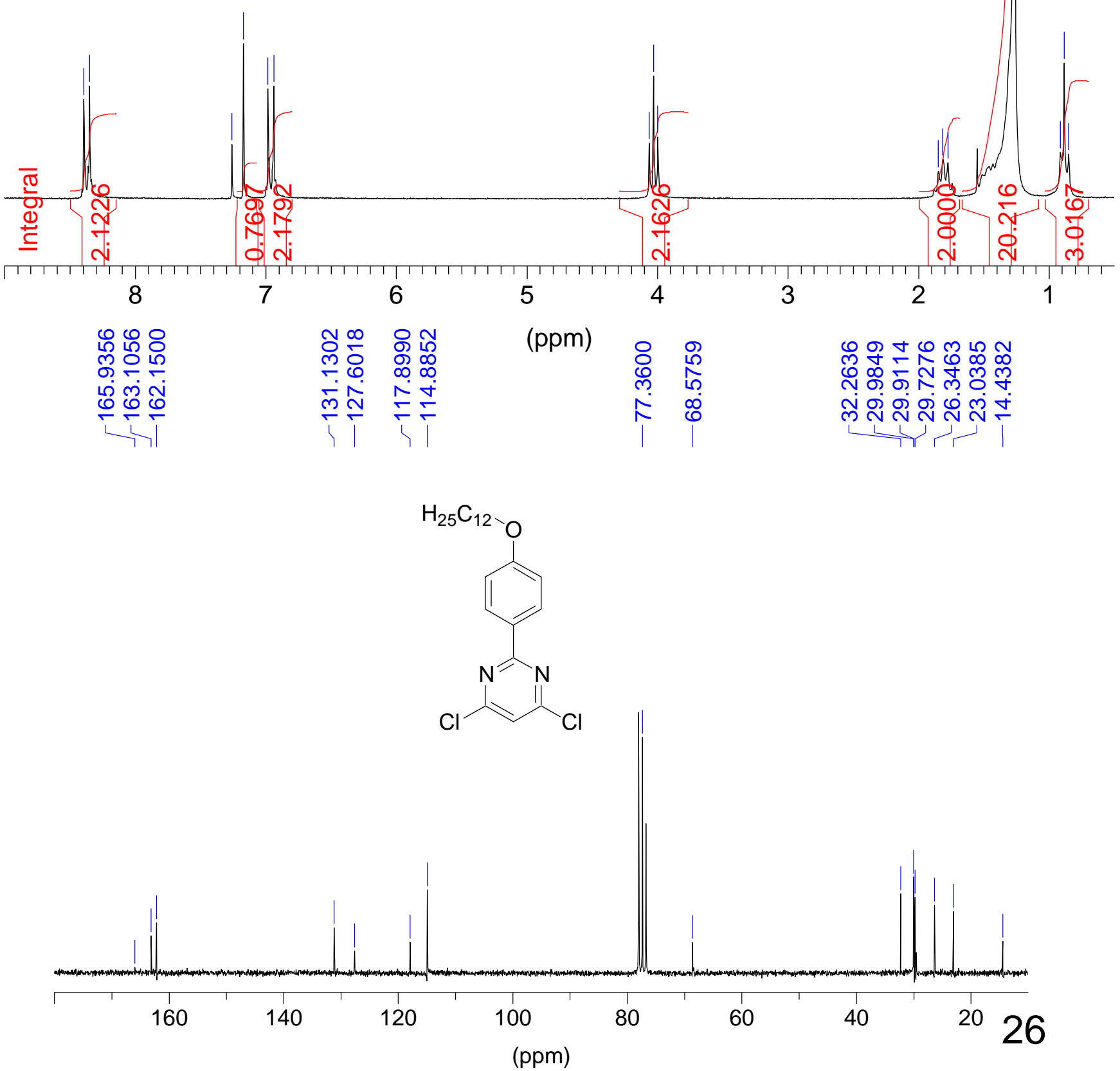

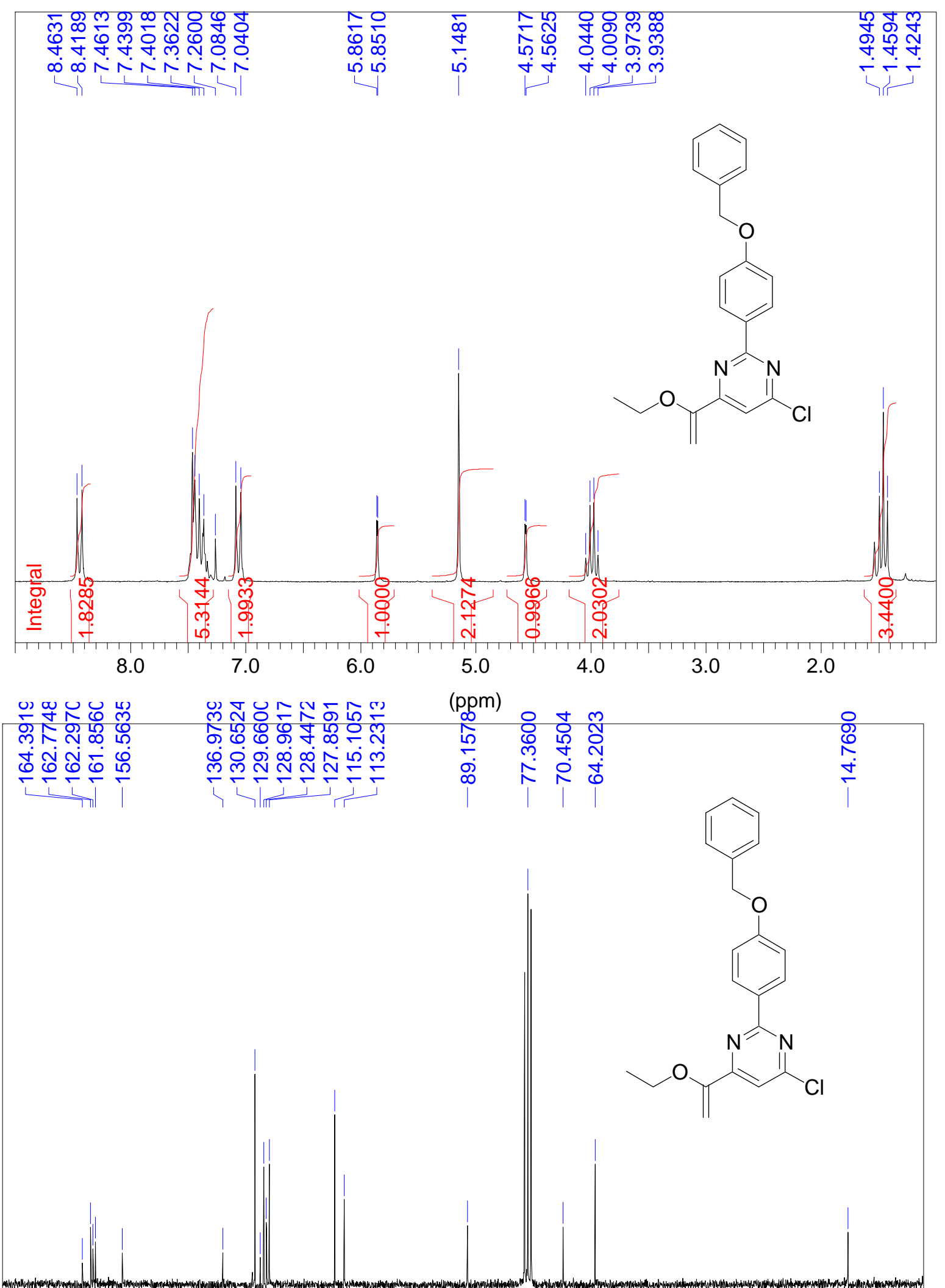


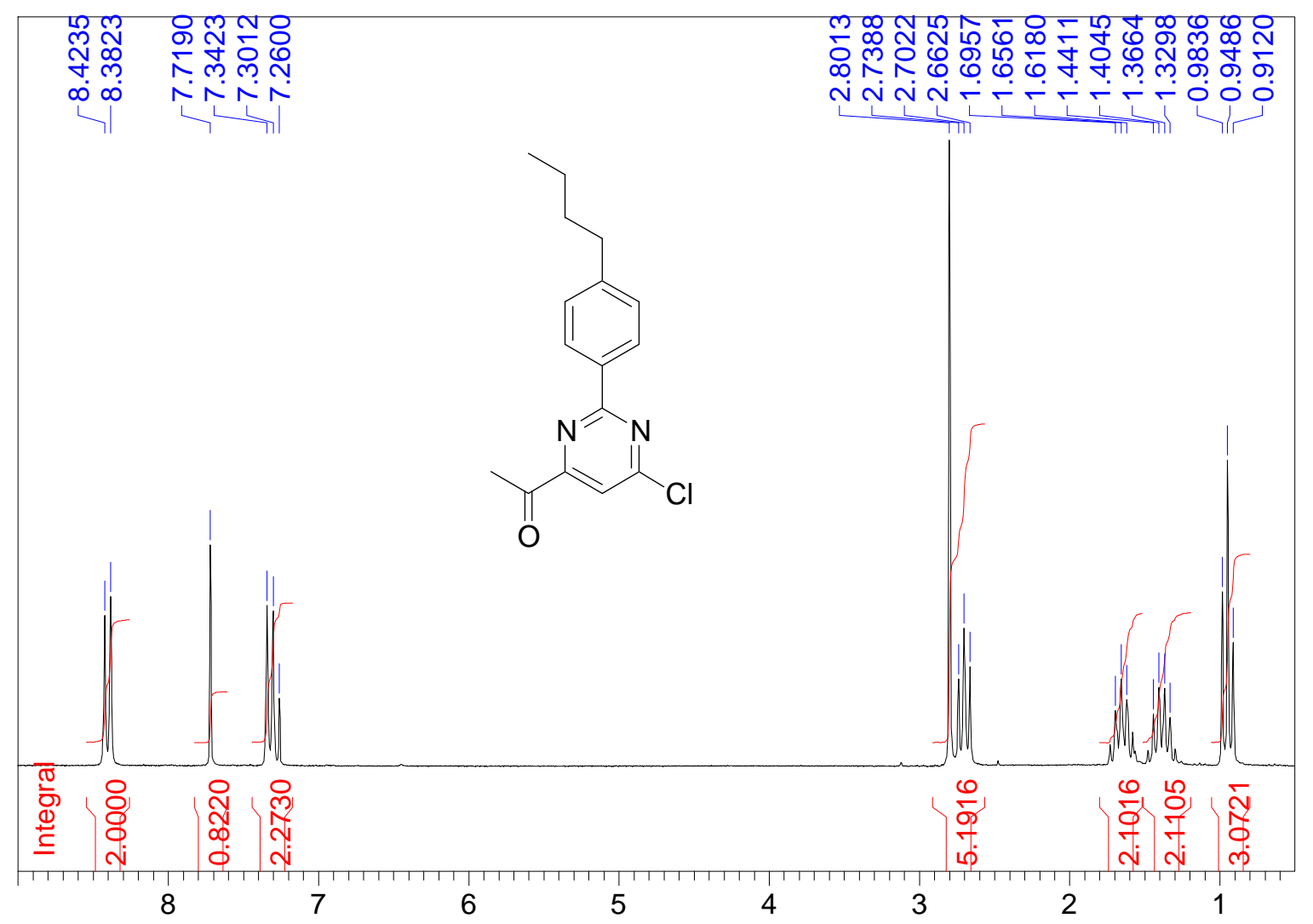

(ppm)

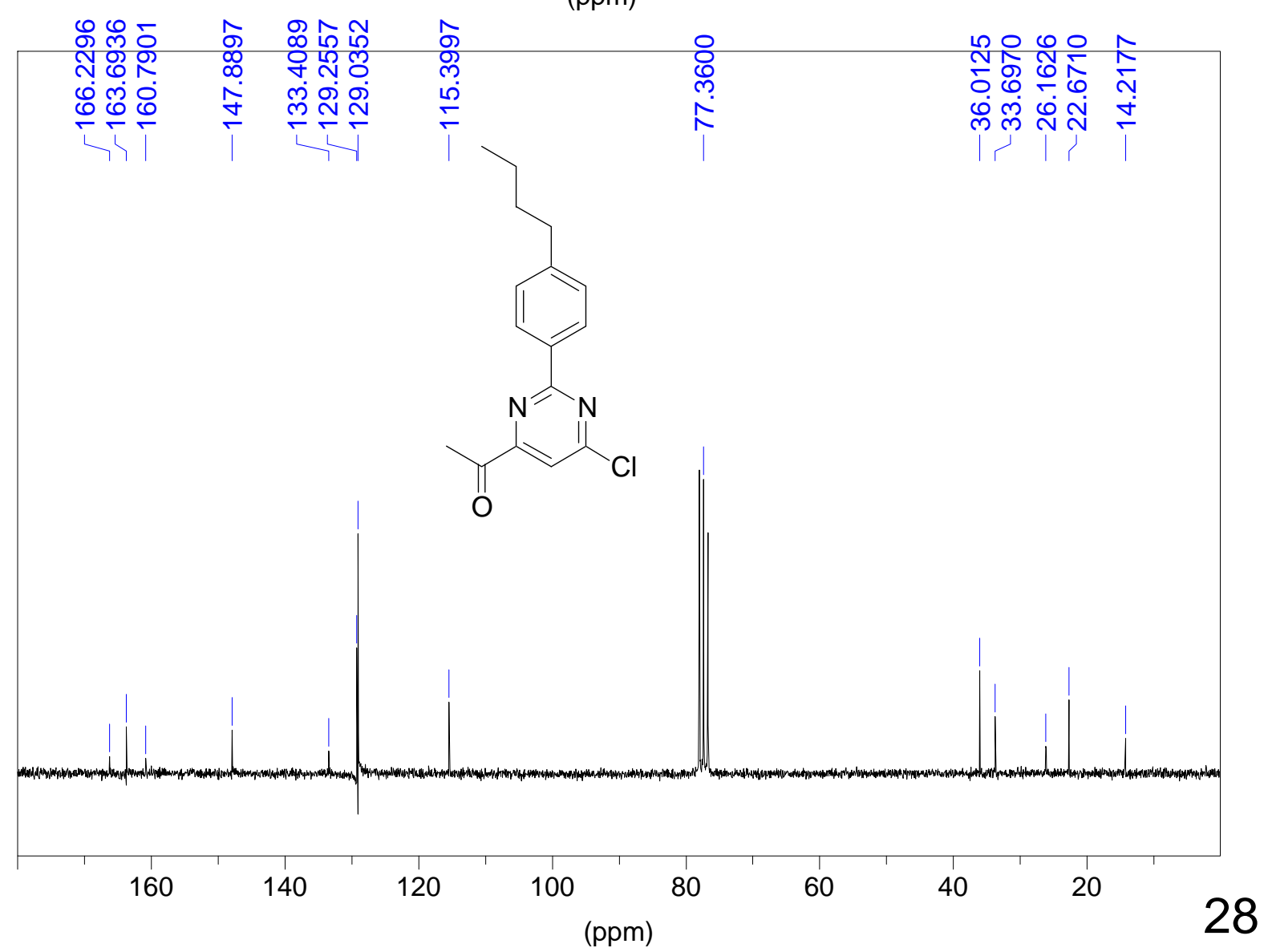




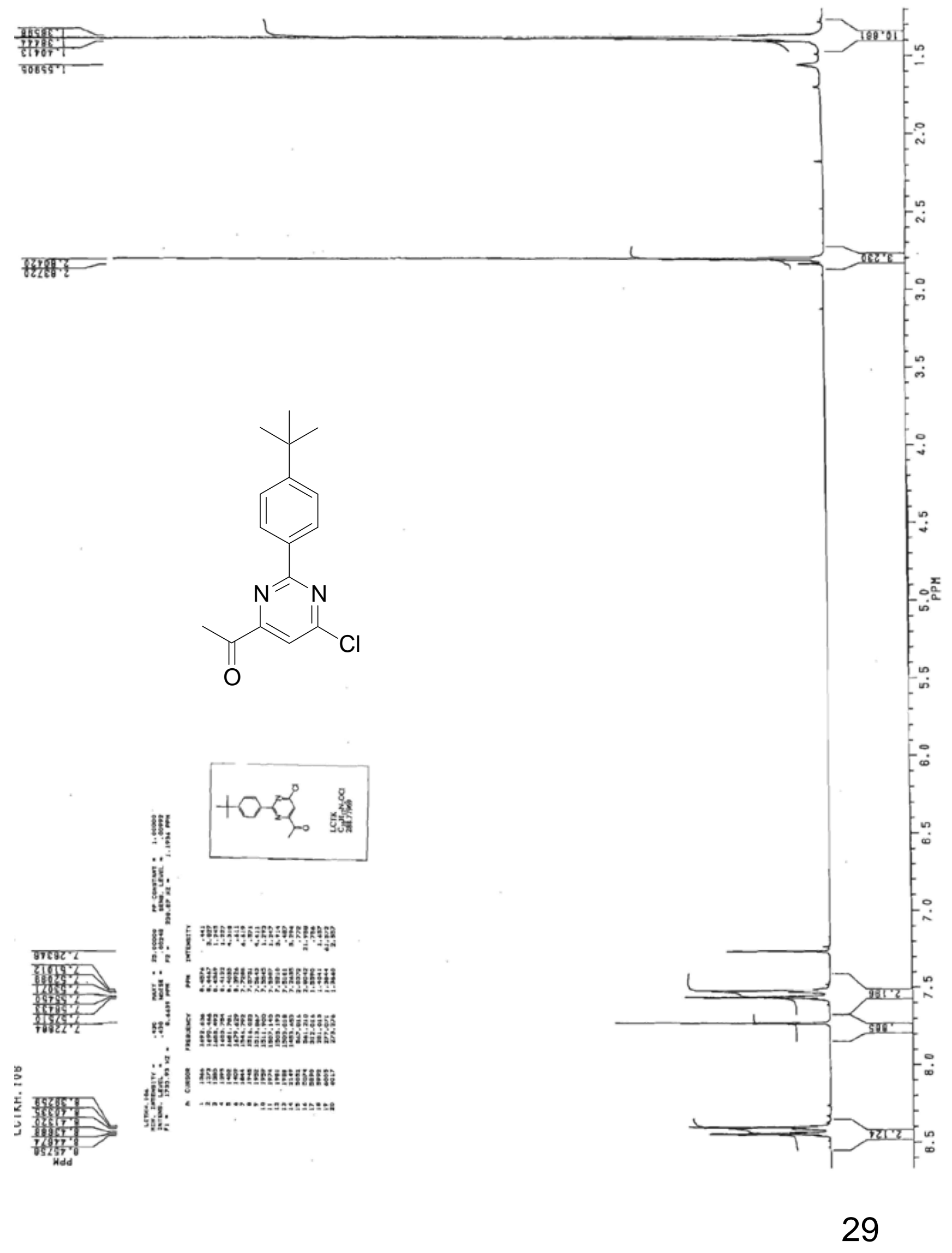




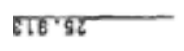

$228 \cdot \sqrt{6}$

हाग्द्रह

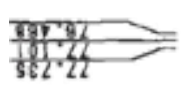

जहा जा।

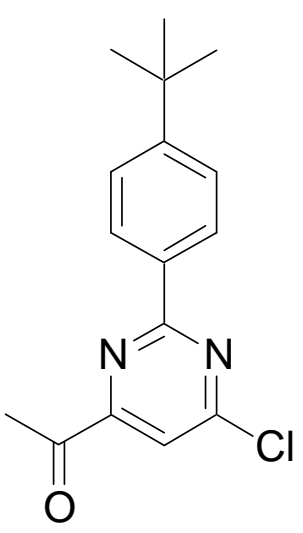

$\frac{200 \text { ड21 }}{600.921}$

डटह हहा

Tार्डst

सहम्श -

रहा होगा

हट० क्षा
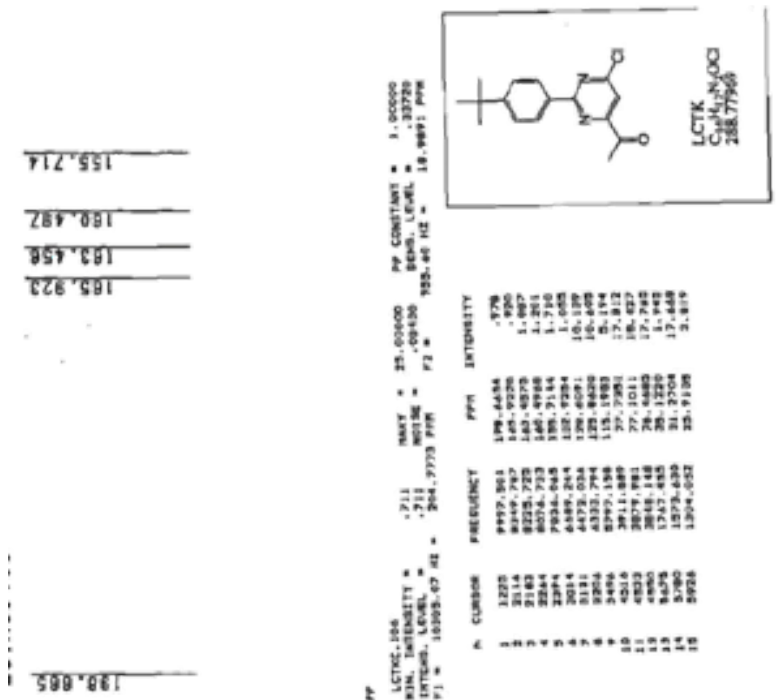

88.

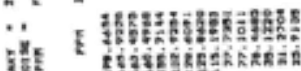

类

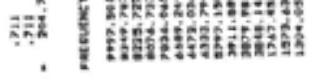

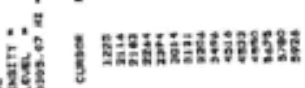

$+\frac{1}{2}=$

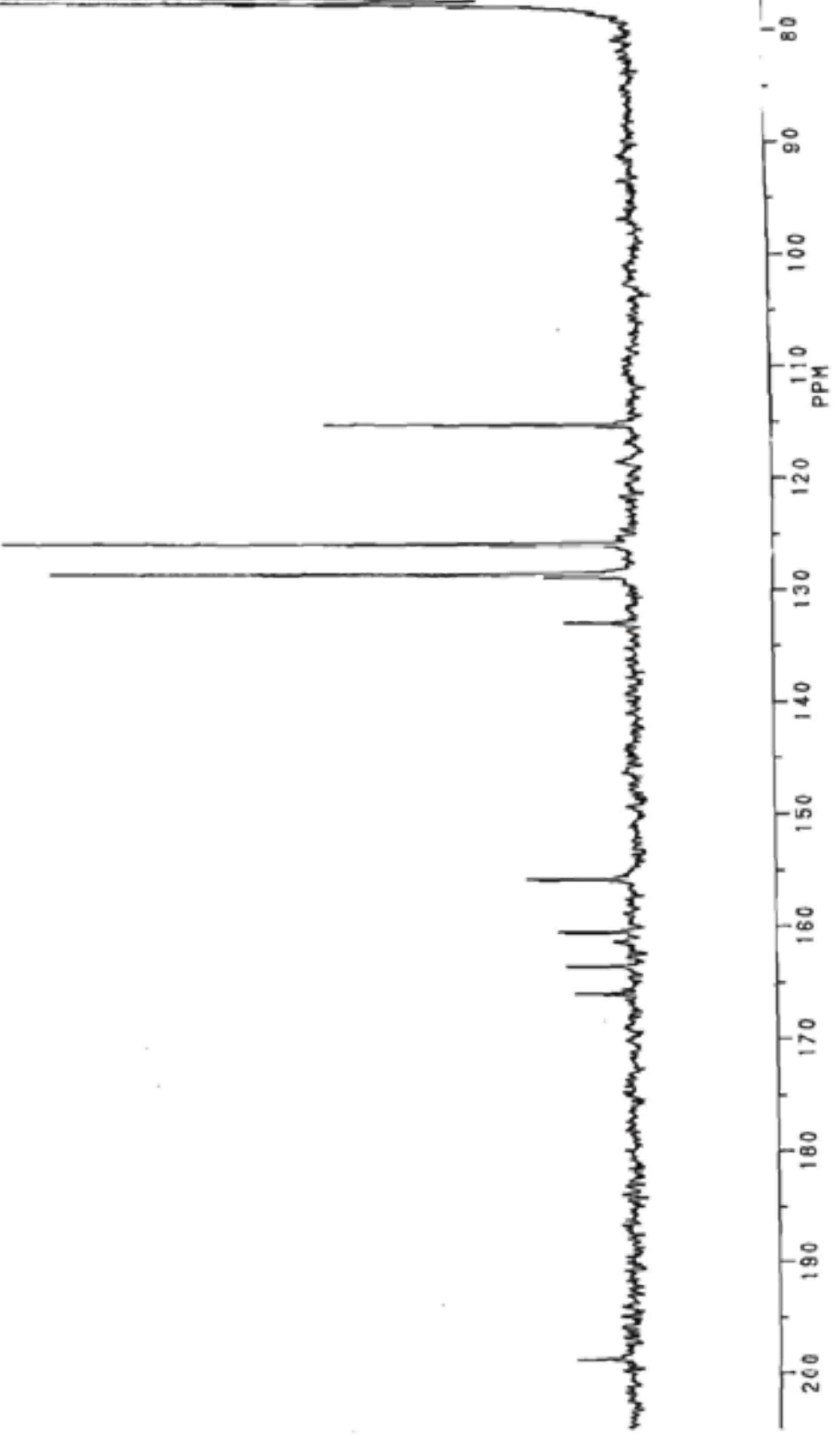




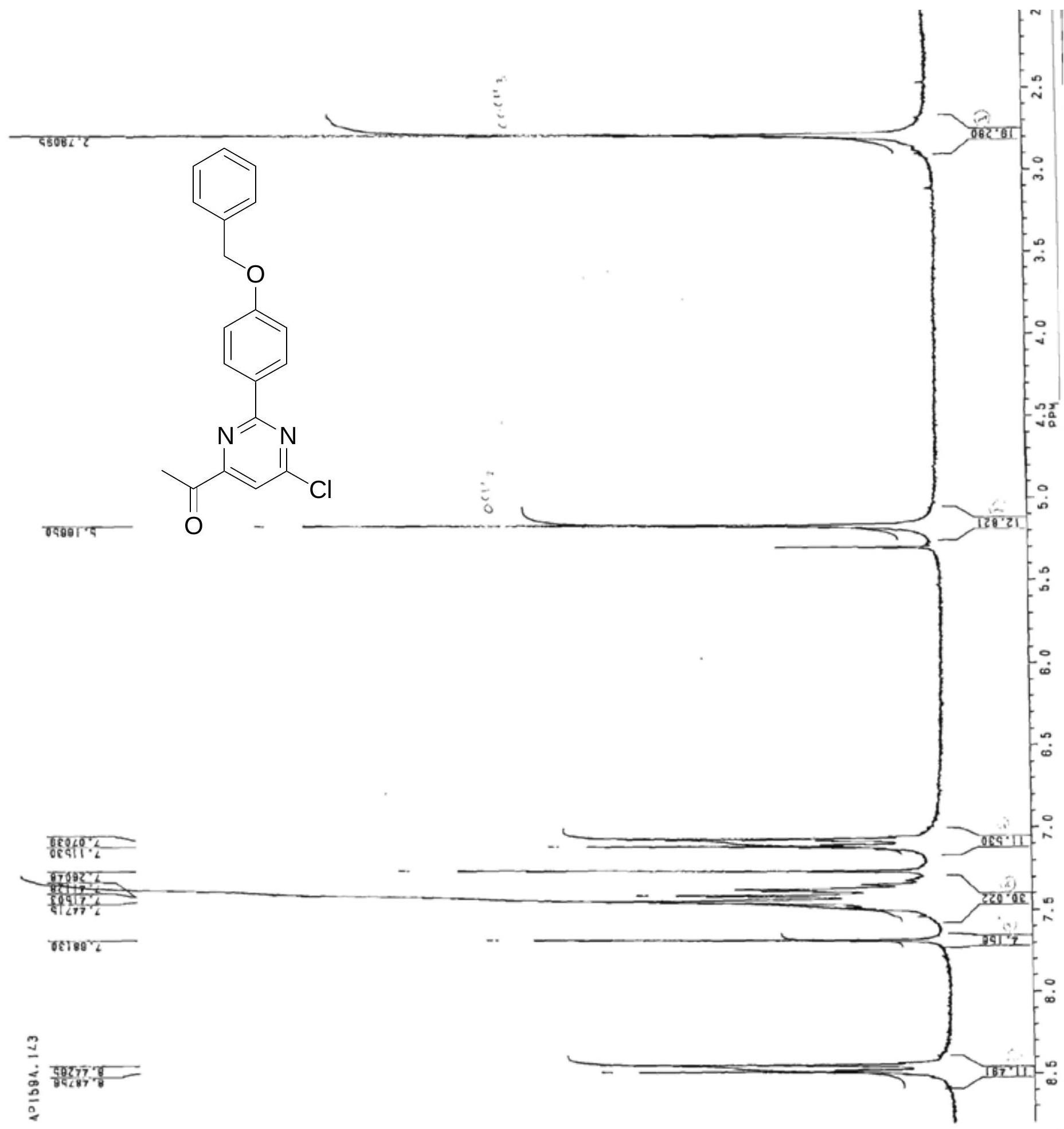




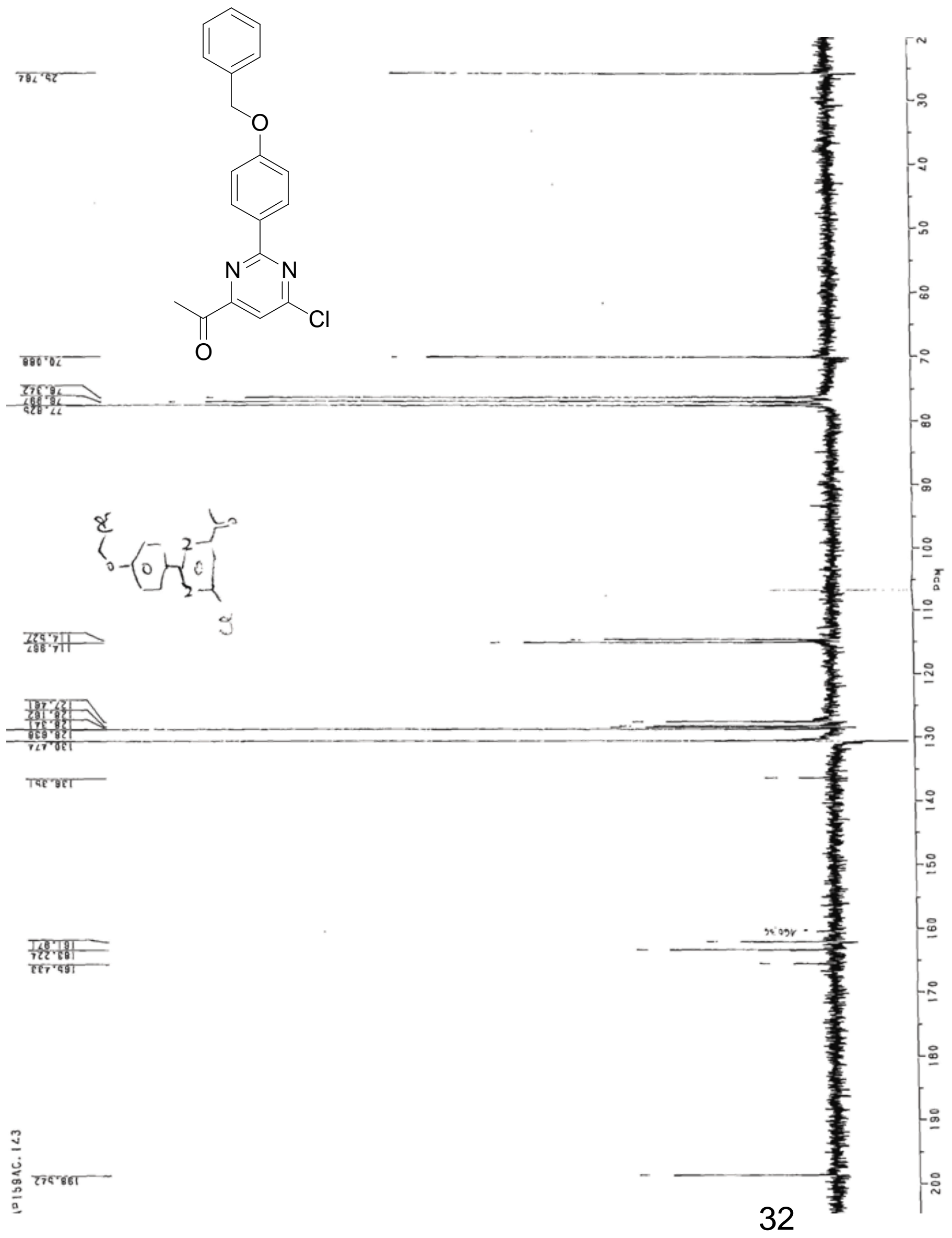




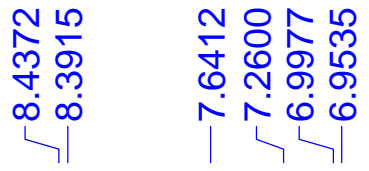

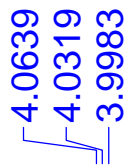

$\stackrel{\substack{N \\ N}}{N}$

ป

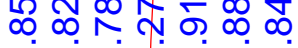

i

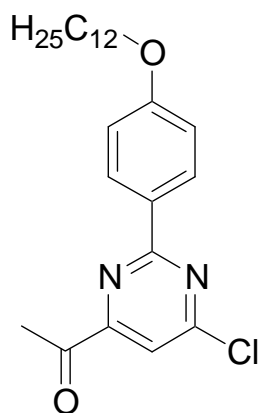

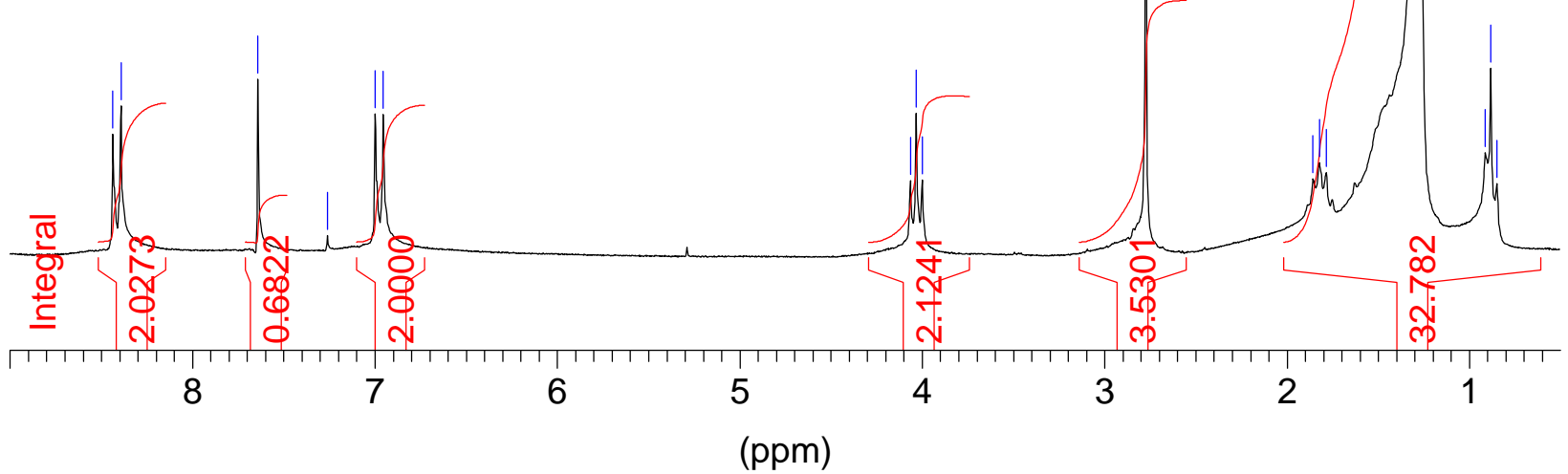

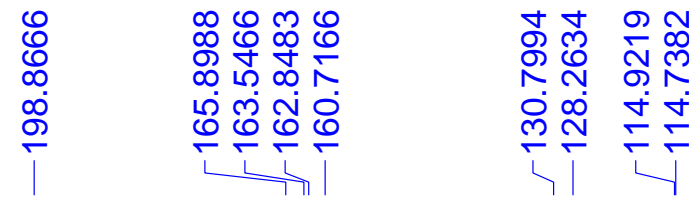

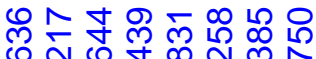
ํํㄴำ กิ

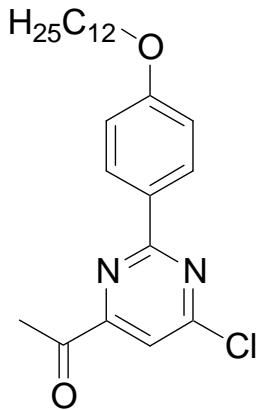

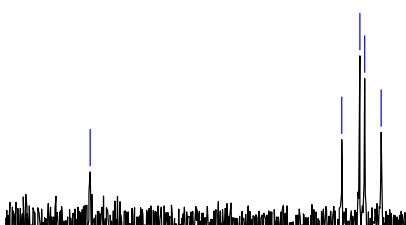




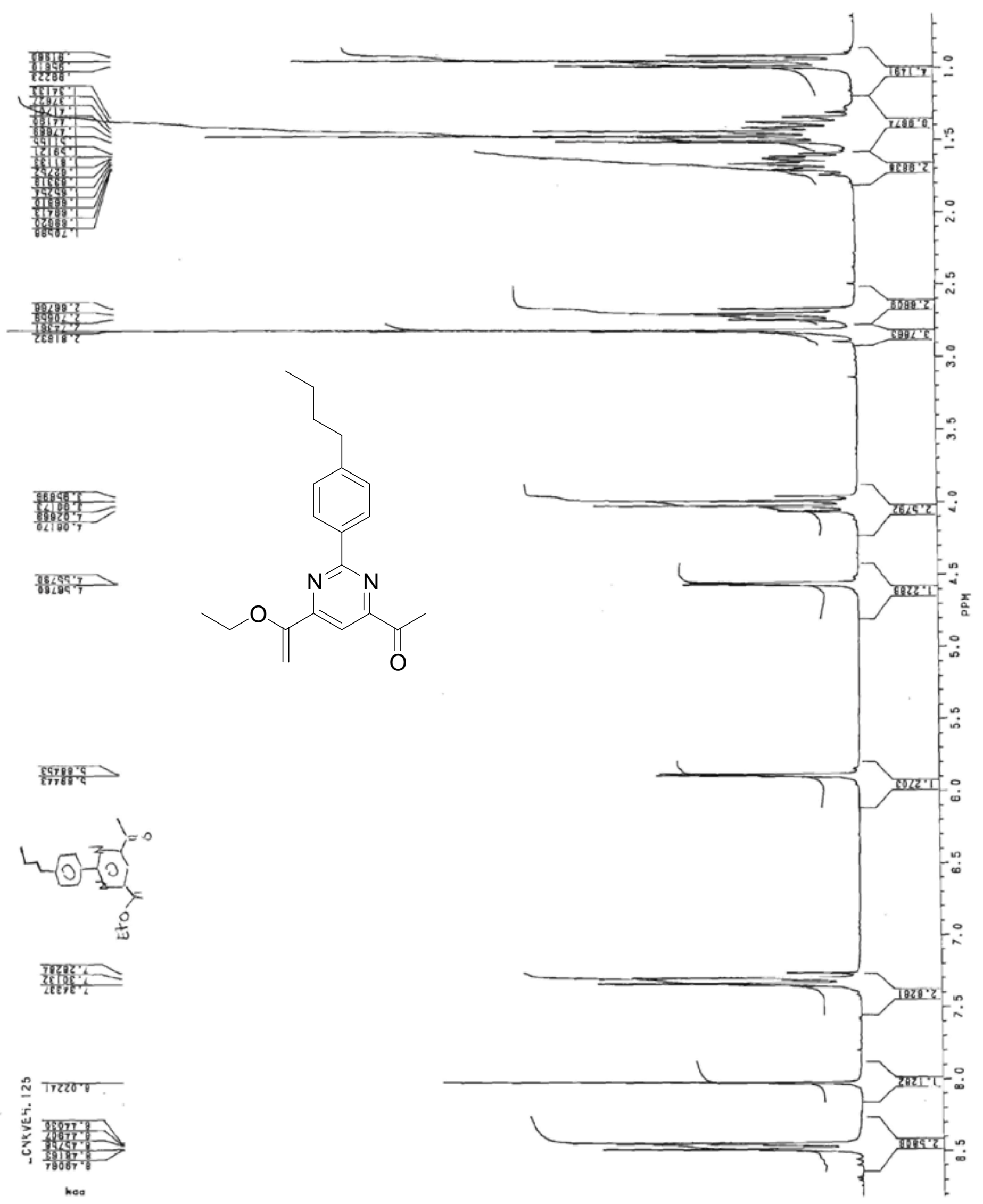


6ettr2 OZ98'sz -

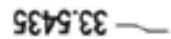
6ILL' $\varsigma$ -

乙SL6 \&9 -

180S' $9 L$

$6 Z E I$

LSLLL

L961'88

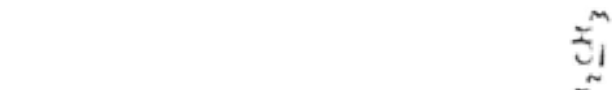

$=1$

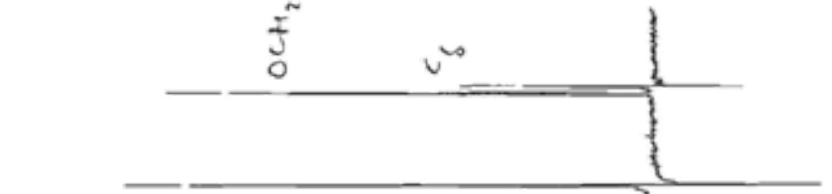

트으

오<smiles>C=C(OCC)c1cc(C(C)=O)nc(C)n1</smiles>

$91<0601$<smiles></smiles>
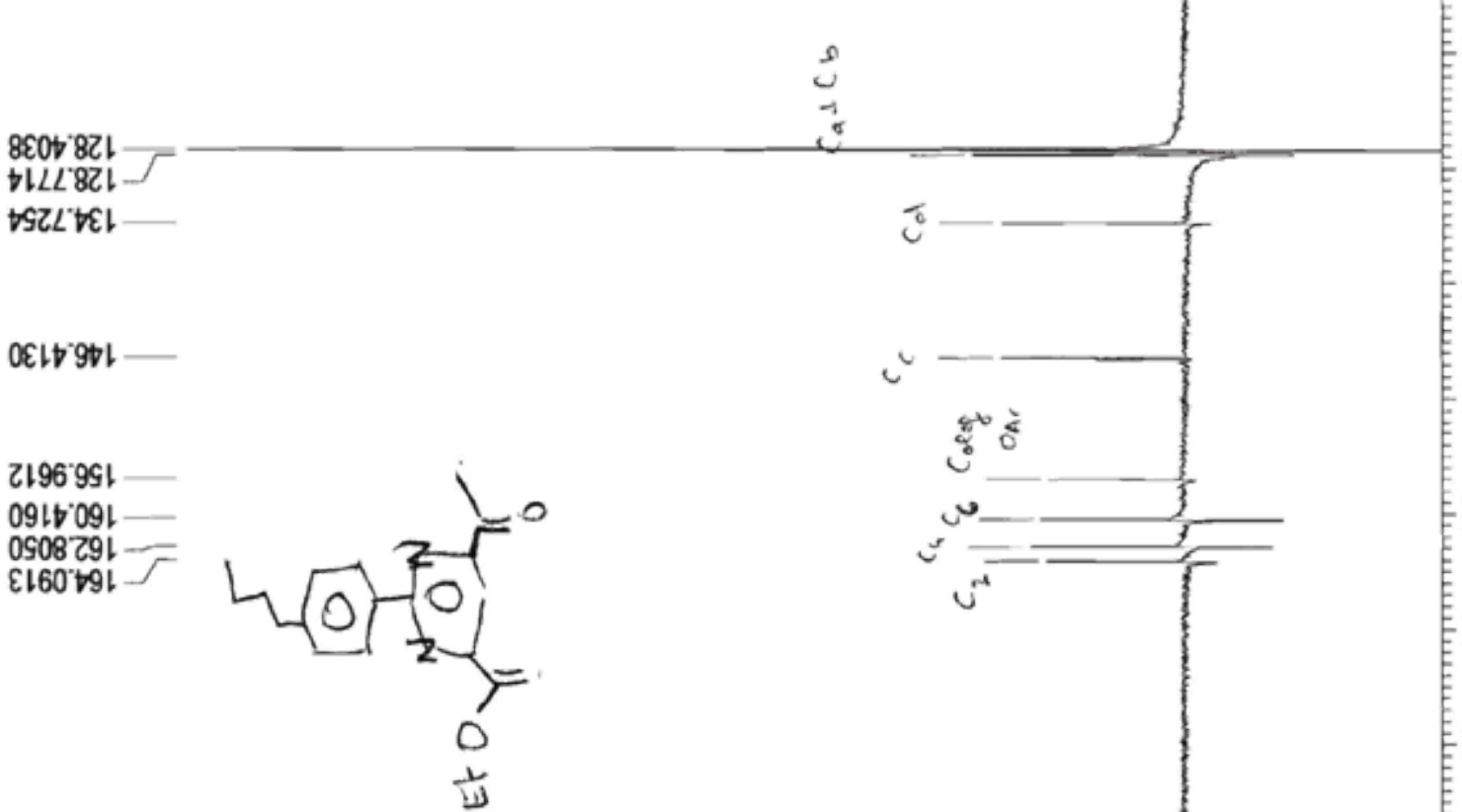


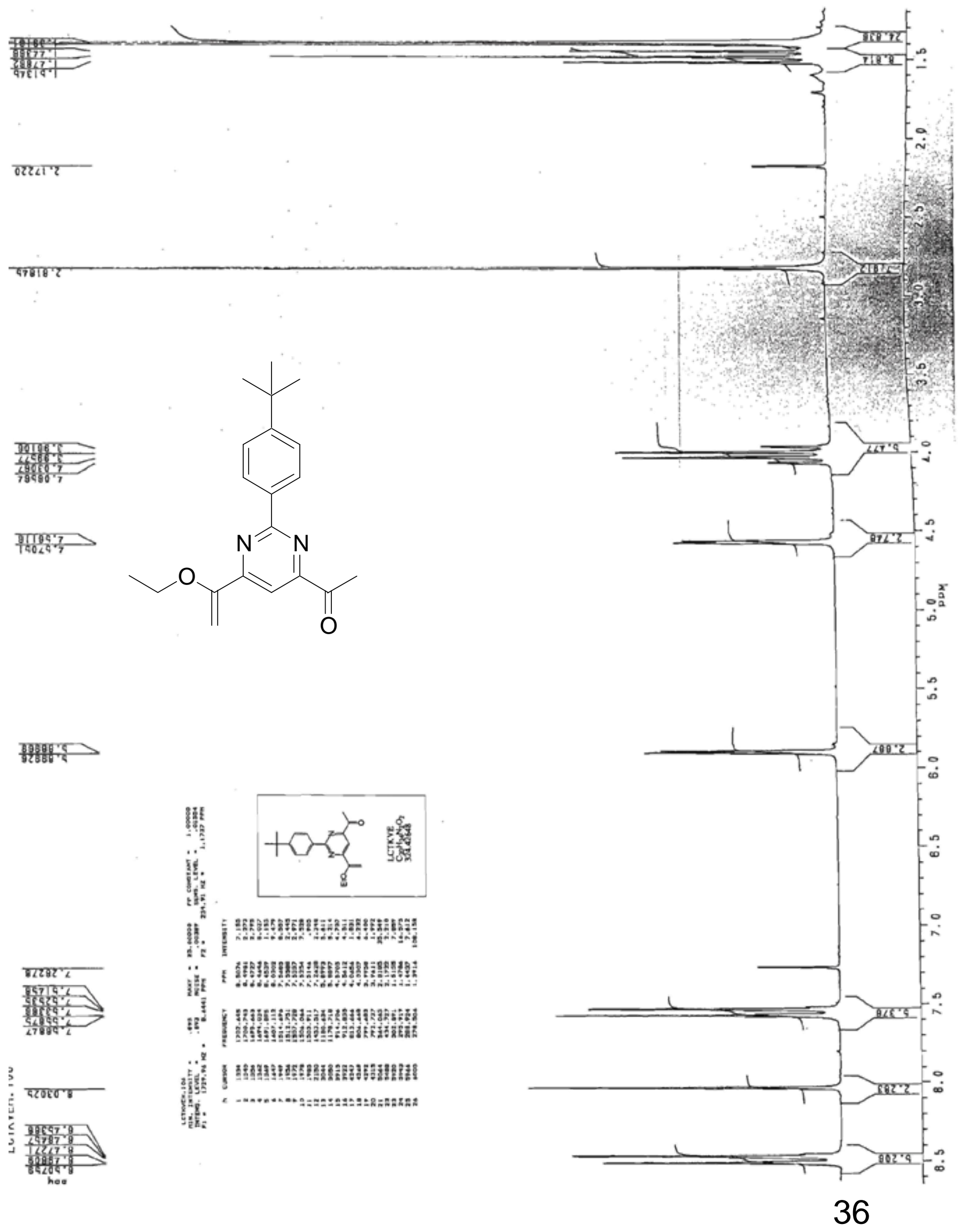




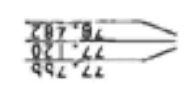<smiles>C=C(OCC)c1cc(C(C)=O)nc(-c2ccc(C(C)(C)C)cc2)n1</smiles>

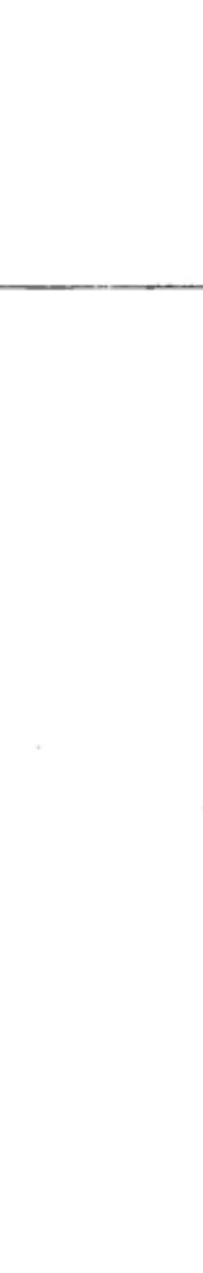

व7द्वरा हार

रत्बा

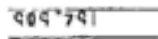
ग्राड द्वा 7470 वा

480790

언
는
는

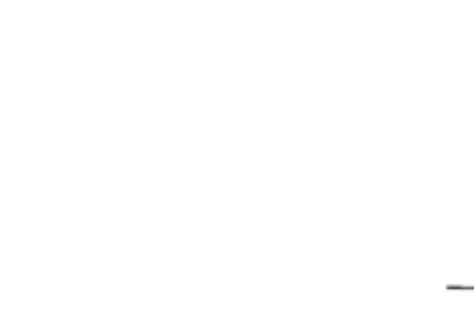

70298

${ }^{960} \cdot 601$
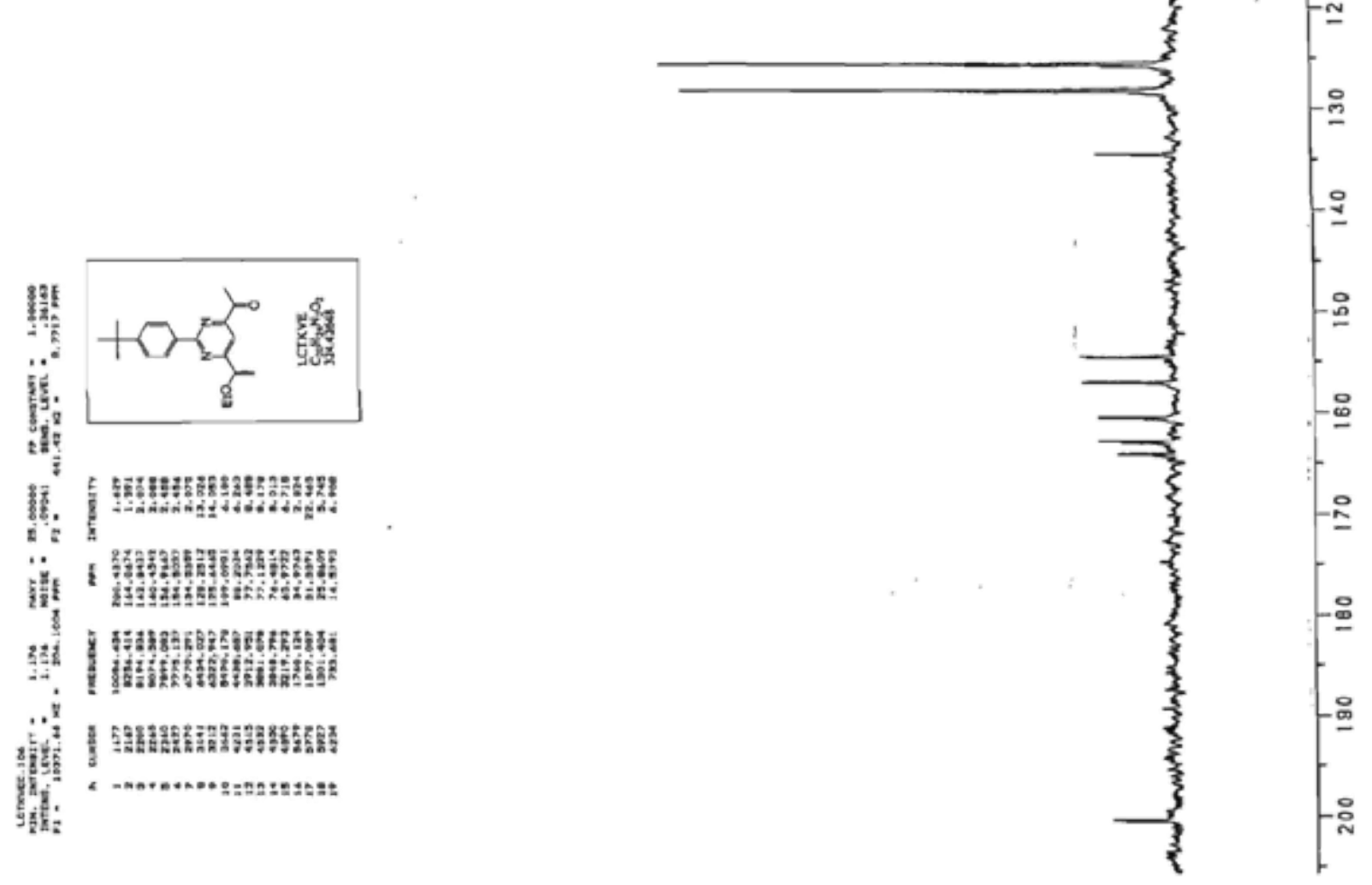


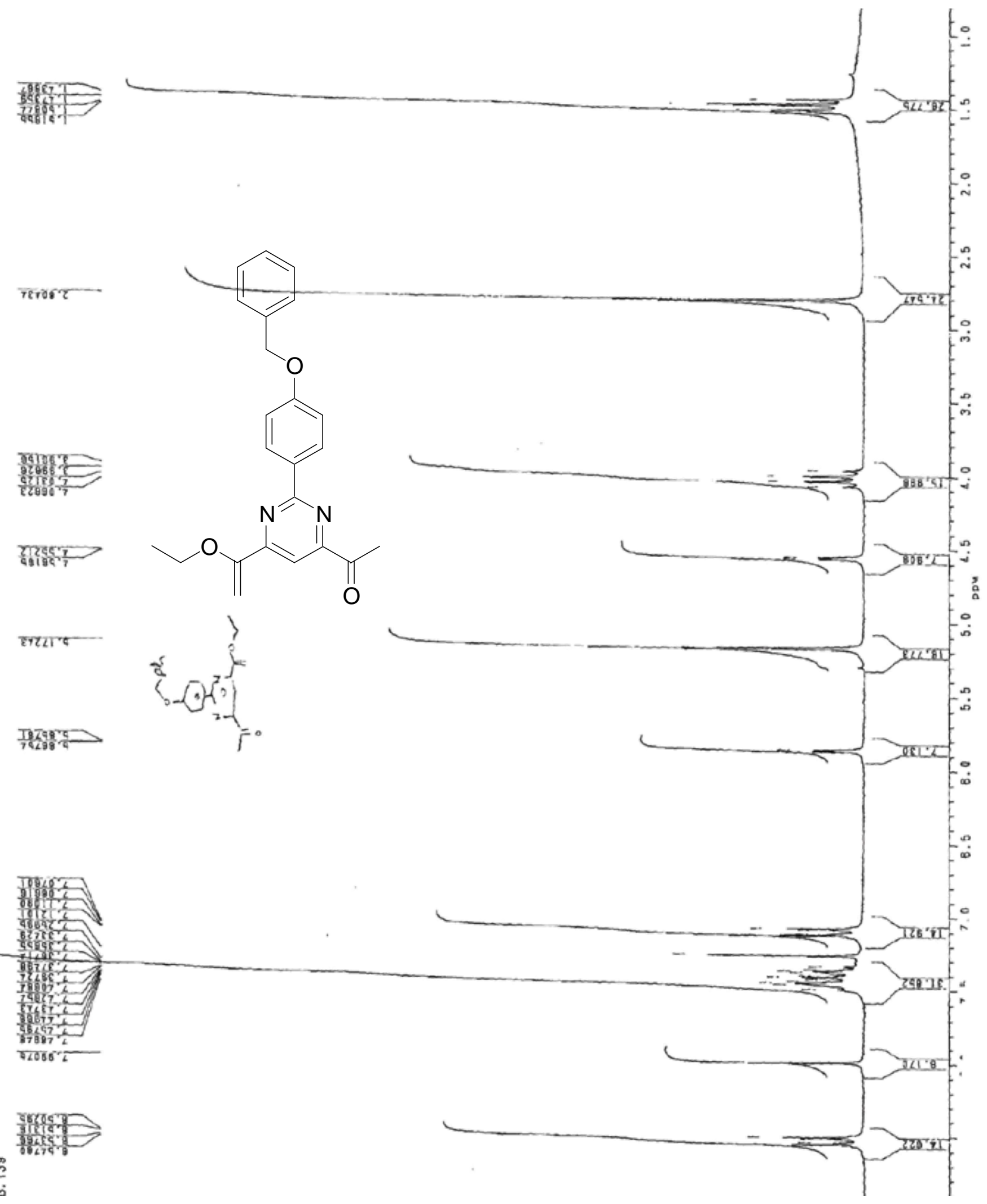



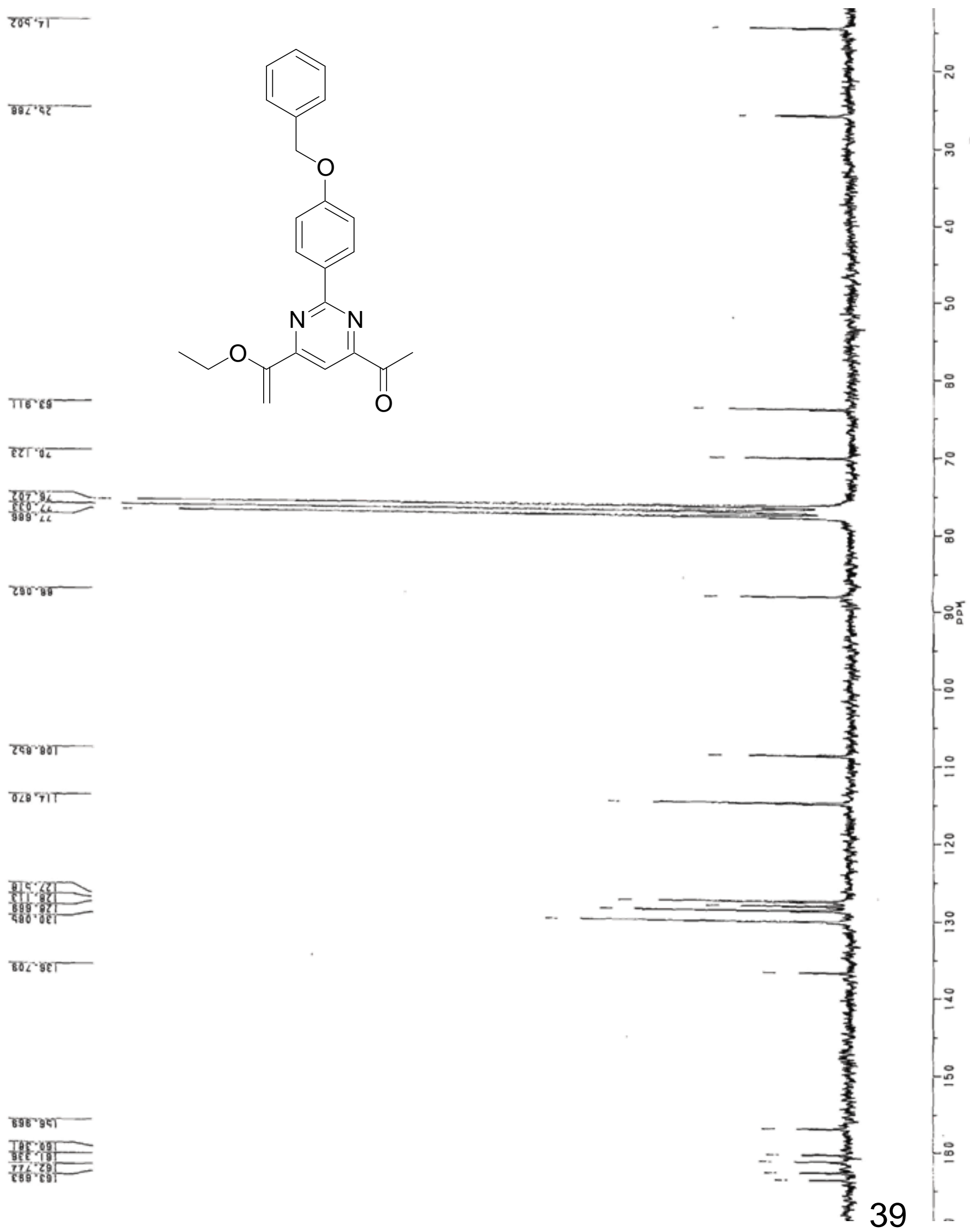
긍유

กิ ๙

$\infty \infty$ N

$\infty$

○

ᄂ

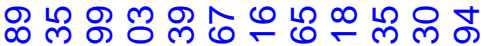

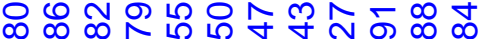
N-i

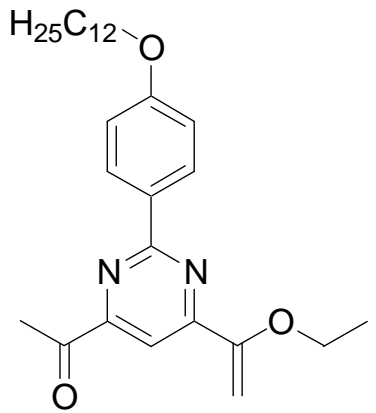

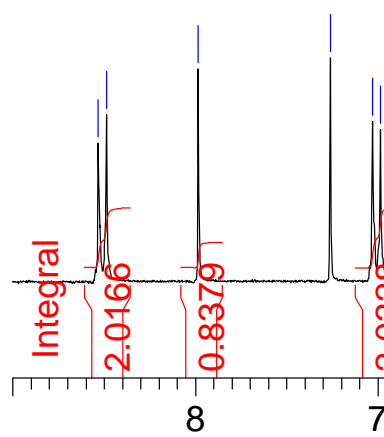

7

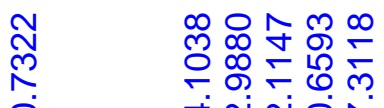

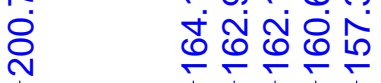

।

$\mathrm{H}_{25} \mathrm{C}_{12} \backslash \mathrm{O}$

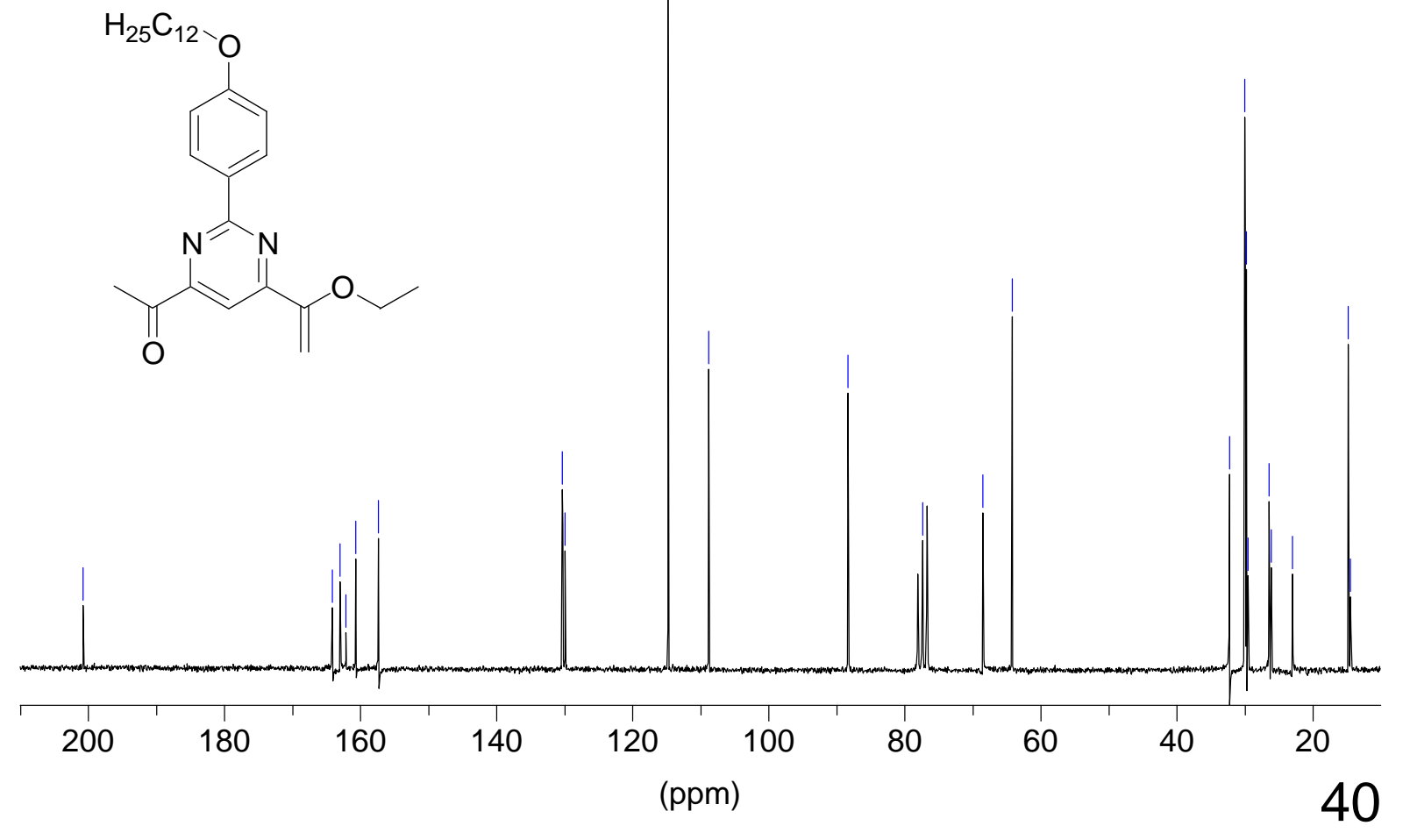

\section{(ppm)}

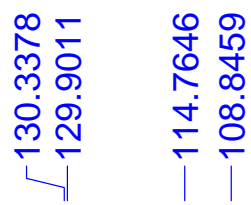

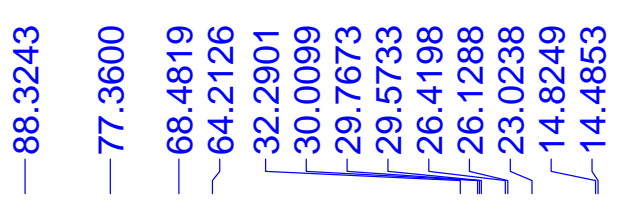




\section{0}

26560

$\varepsilon+66^{\circ} 0-$

$99+\varepsilon ' I\urcorner=$

ZE\&E $I$

$82 Z H^{\circ} \mathrm{I}$

6LS'I

$8 \angle E 9^{\circ} \mathrm{I} \longrightarrow$

2299' I -

06L9' I

ILILI

0069 '

$182 L ' Z-F$ $2994 \mathrm{~L}-$

†9E $\mathrm{Z}$
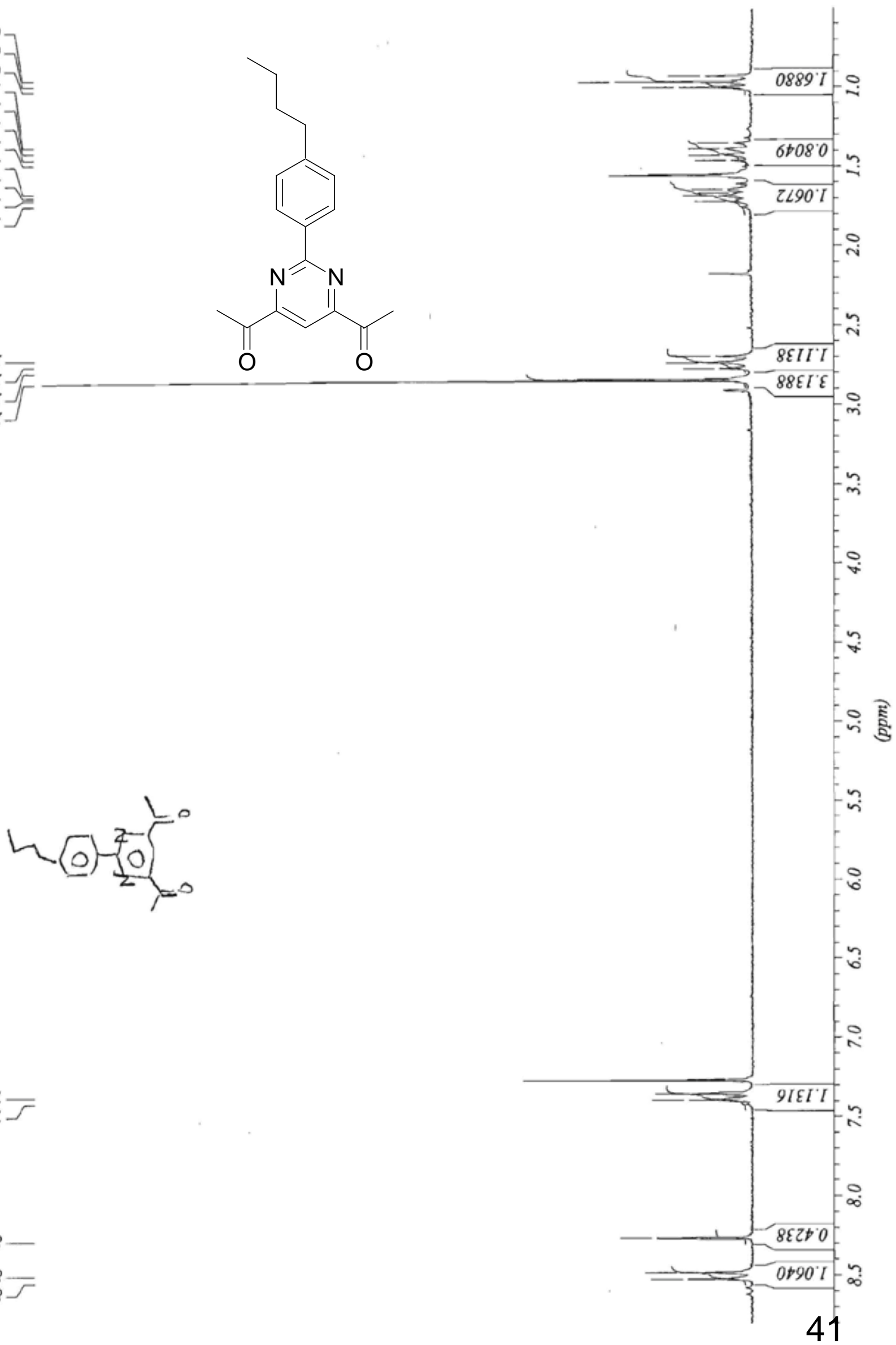

$\rightarrow S t \varepsilon L$

$998 \varepsilon^{\circ} L$

ELSZ:

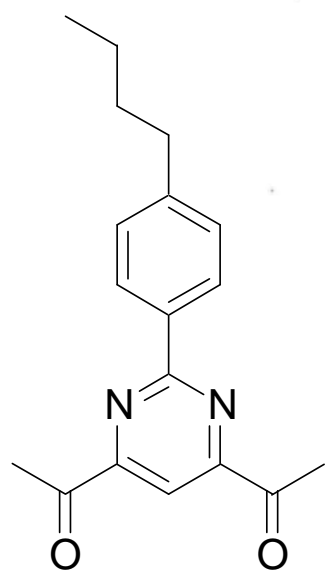


Stoczz-

$6 Z L S Z$

$989 \varepsilon \varepsilon \varepsilon-$

SL†9'SE
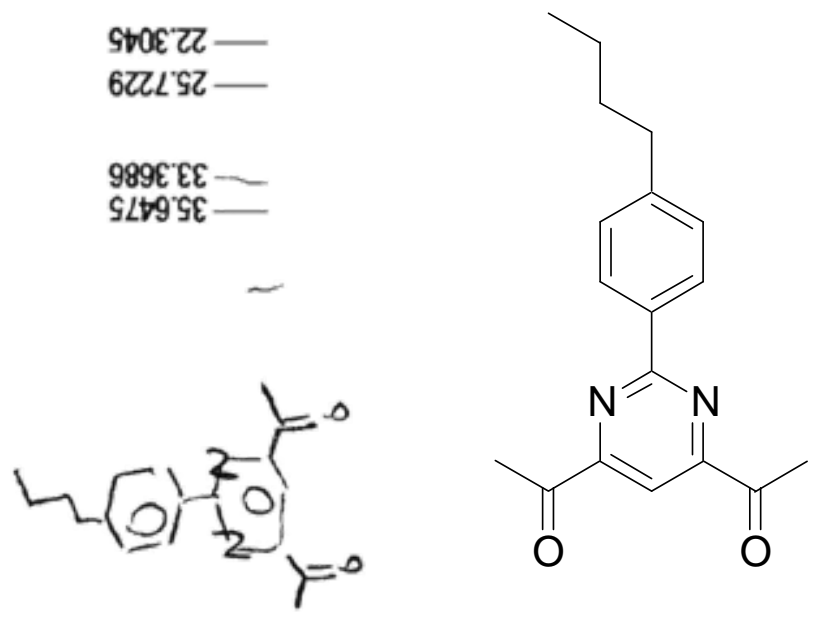

ISLE $9 L$

$0000^{\circ} L \frac{}{-}$

SZOEOLL-

$1 \rightarrow Z 78 Z$

$\angle 8 E 6^{\circ} 8 \mathrm{ZL}$.

†089"हย।

$\varepsilon \angle O Z \angle t b-$

OZLZ $เ 9 !-$

$682 \varepsilon \div 91-$ 


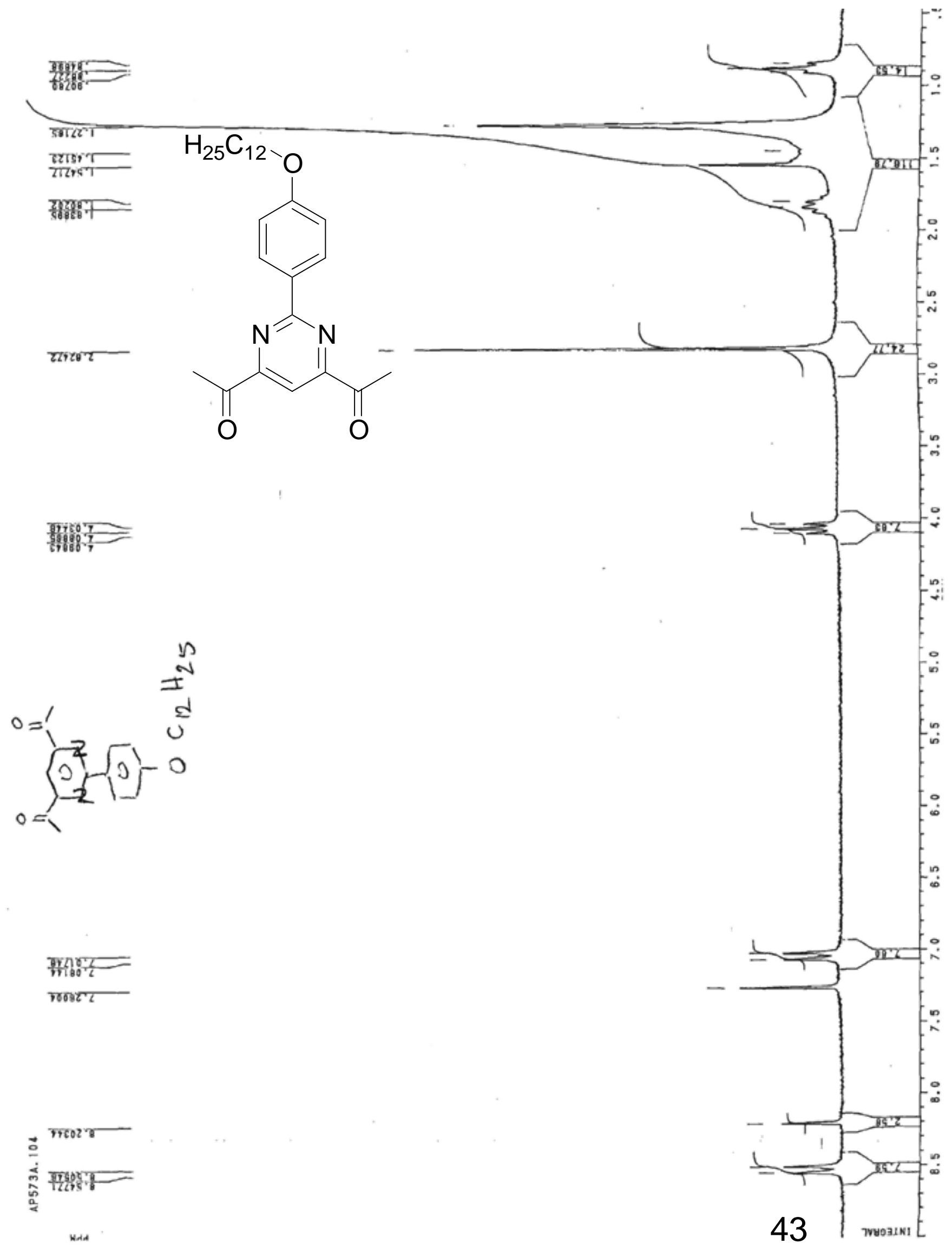




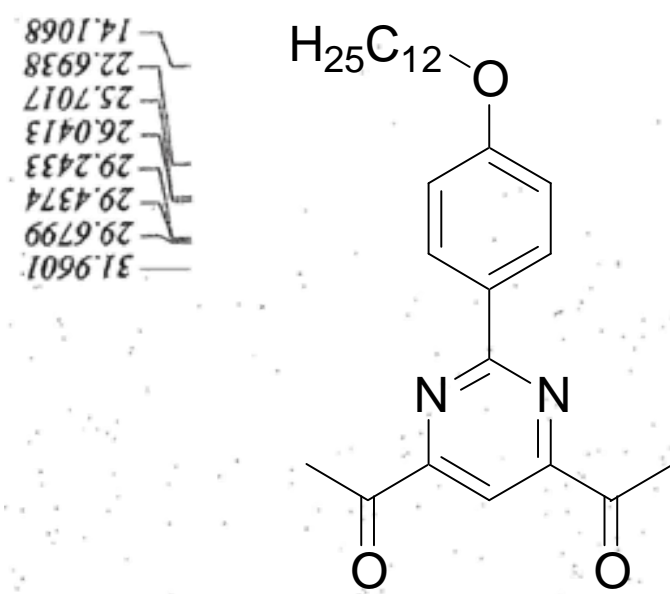

$\$ \angle 6289$

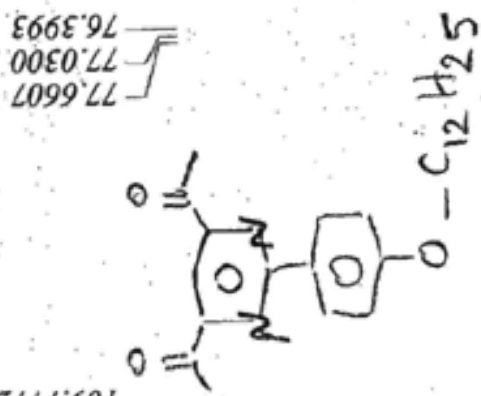

ZLLL6OI -

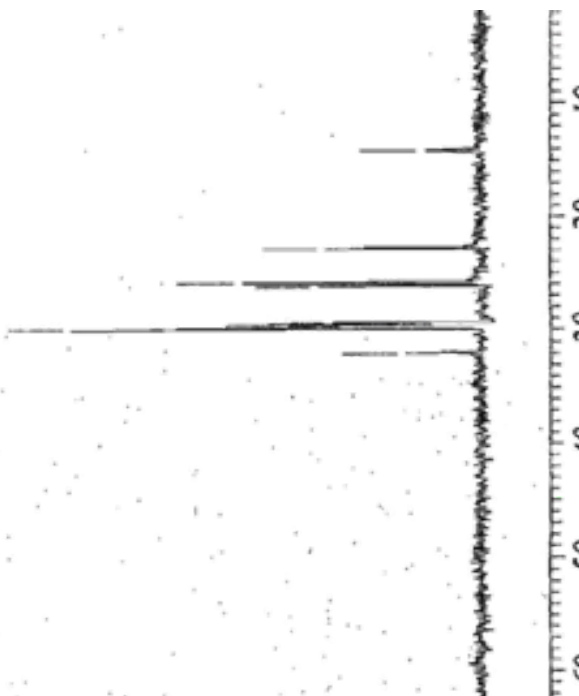

ZLLQ: $\$ I I-$

$86+9.821-$

$8102^{\circ} 0 E 1$ -

OISZ'19I-
E8IE'Z9I-

ZZEI'SOI

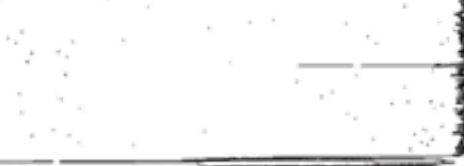

E요

E

영

8 
$\varepsilon \pitchfork \tau \varepsilon \cdot I$

कбSE $T=$

6SLS $I$

9SI9 $I \perp$

ZLE9 $Z$

DSZ9 $Z-2$
$0 Z T L 2$

$I \angle \mathcal{E} \mathcal{E}^{\circ} \mathcal{-}$

$3_{\mathrm{nBu}}$

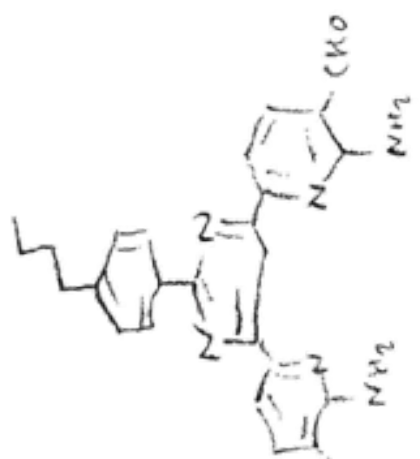

$\varepsilon+\varepsilon 60^{\circ}$

$x$
0

$506 I^{\circ} \mathrm{L}$

DOLE $\angle I T-$

$\varepsilon \varepsilon \varepsilon L{ }^{\prime} L-$

$1+662 \mathrm{~L}$
22808

$60928-$
$900 \varepsilon 8-\Gamma$

EL6t 8 -

$58 E 58$

42268
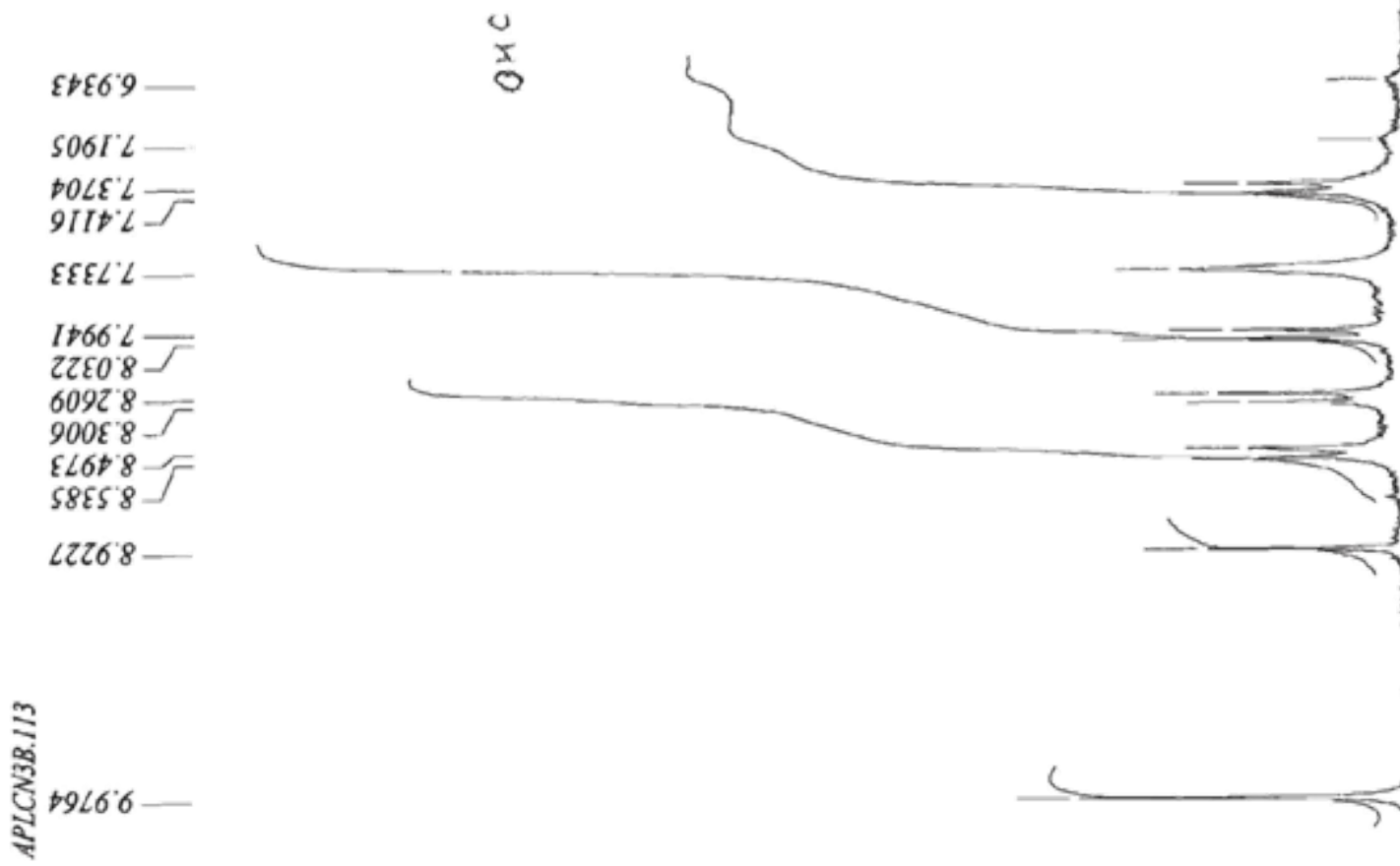
$\angle 686^{\circ} 60 I-$

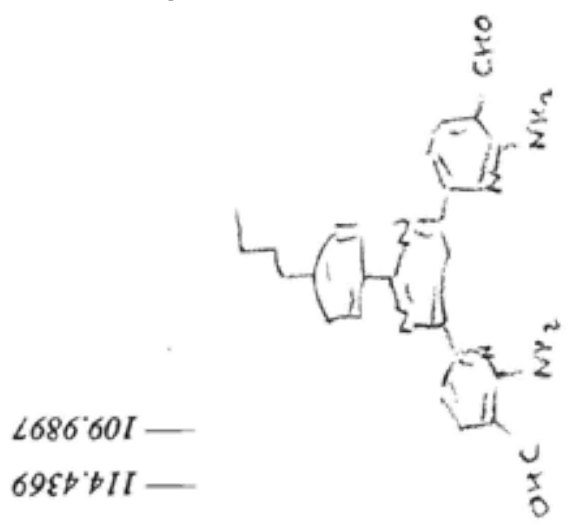

$69 \varepsilon \mapsto " b I I-$

$0296^{\circ} \angle 2 I-$

EEIS" $8 Z I-ᄂ$

$3_{\mathrm{nBu}}$

$00 s S s+I-T$

OLL9'SDI-

$\varepsilon \angle 8 L ' S D I-$

$\left.9 \angle 68^{\circ} S D I\right]$

$\angle Z 6 G=9 s$

$S L I Z \angle S I-]$
$\left.\varepsilon Z+8^{\circ} \angle S I\right]$

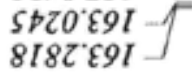

ปิ 
$3_{\text {tertBu }}$

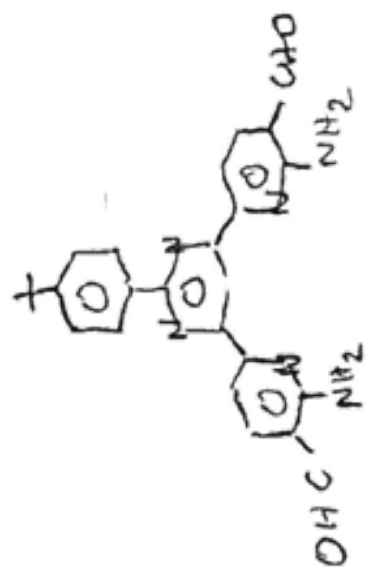

0๕98.9

S6SZL -

$0999^{\circ} L$

$\angle 809^{\circ} \mathrm{L}-$

$0966^{\circ} \mathrm{L}$

เ

SSt:8

12818-

$0<\angle 98$

L6เ9'8

9LOL 6

0

D

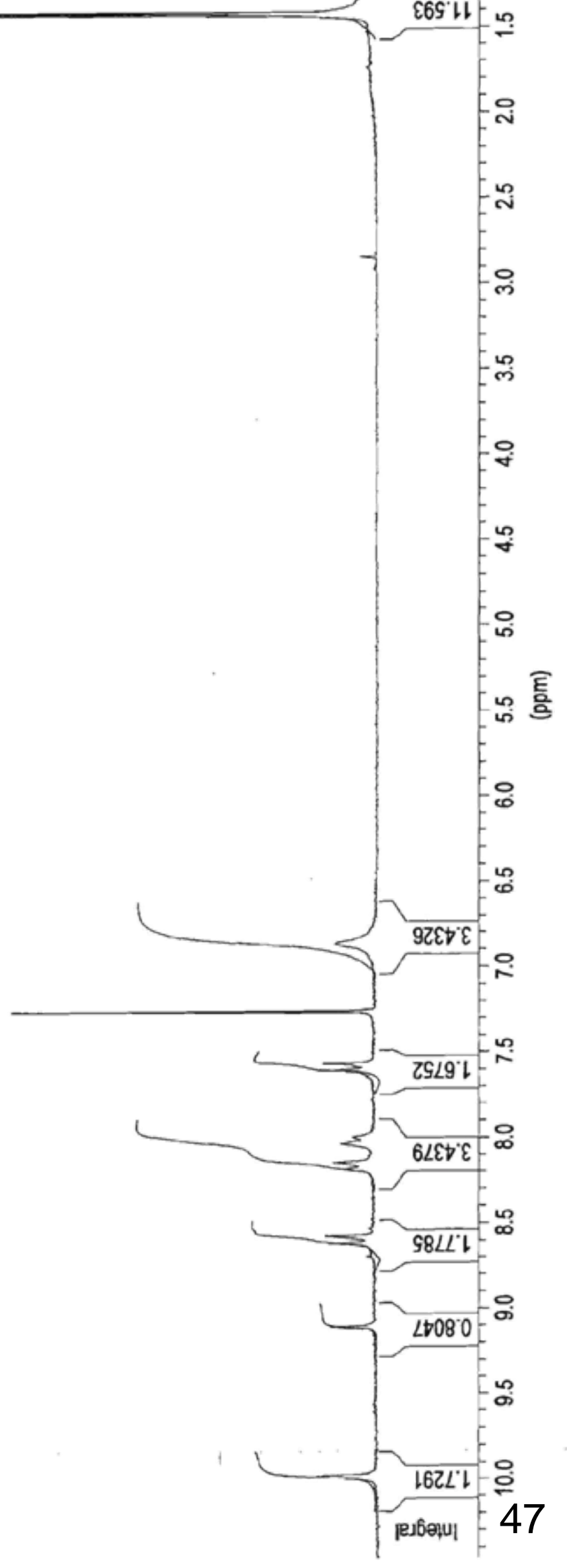


$3_{\text {tertBu }}$

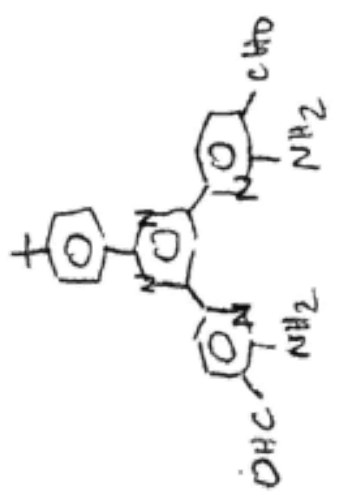

$1800 \mathrm{OHL}-$

8978 ' IL

SS9Stทト -

ZI8t'GZL -

S888 $\angle Z 1-$

เย90"เยเ -

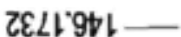

S9ד6 $\varepsilon S$

0L66 951

ZL8L'LSI -

$62700^{\circ} \mathrm{Il}$

ESSE

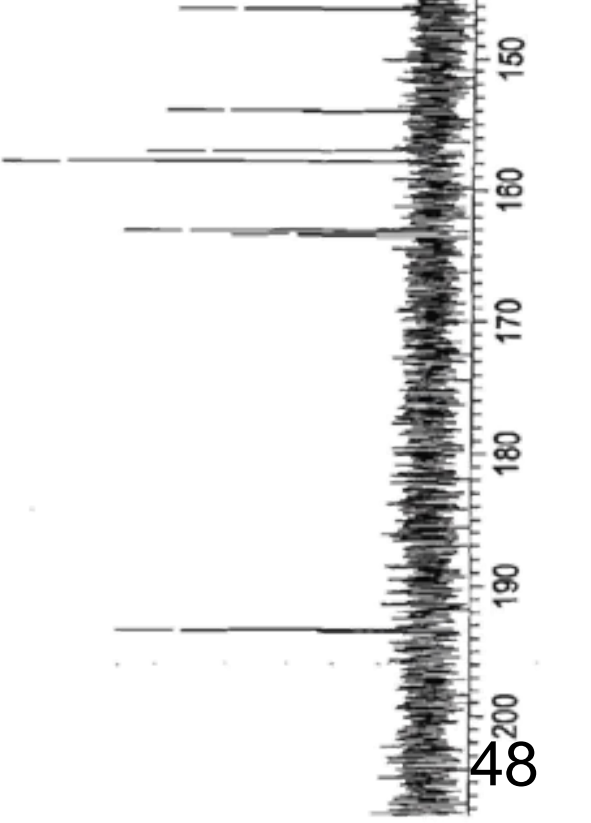




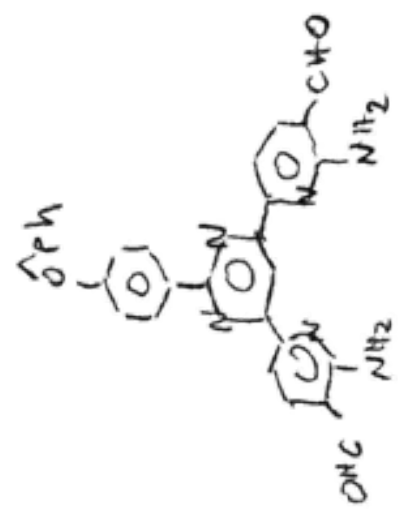

$3_{\mathrm{OBZ}}$

$0 I \varepsilon Z ' S$
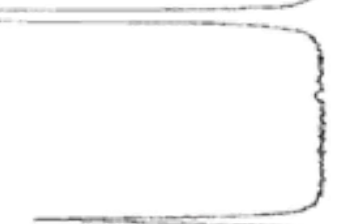
$3_{\mathrm{OBz}}$

(2)

$9960011-$

$+115+11$

$08+6^{\circ}+I I \div$

$+\varepsilon 6 L 621-$

$+289^{\circ} 9 \varepsilon 1$

$\angle Z b I \cdot 9+I-$

9ZE9'\$SI -

$+6+\varepsilon^{\circ} \angle S I$

$91 E 6^{\circ} \mathrm{LS}$

ऽ68609

IILI $E 9 I$
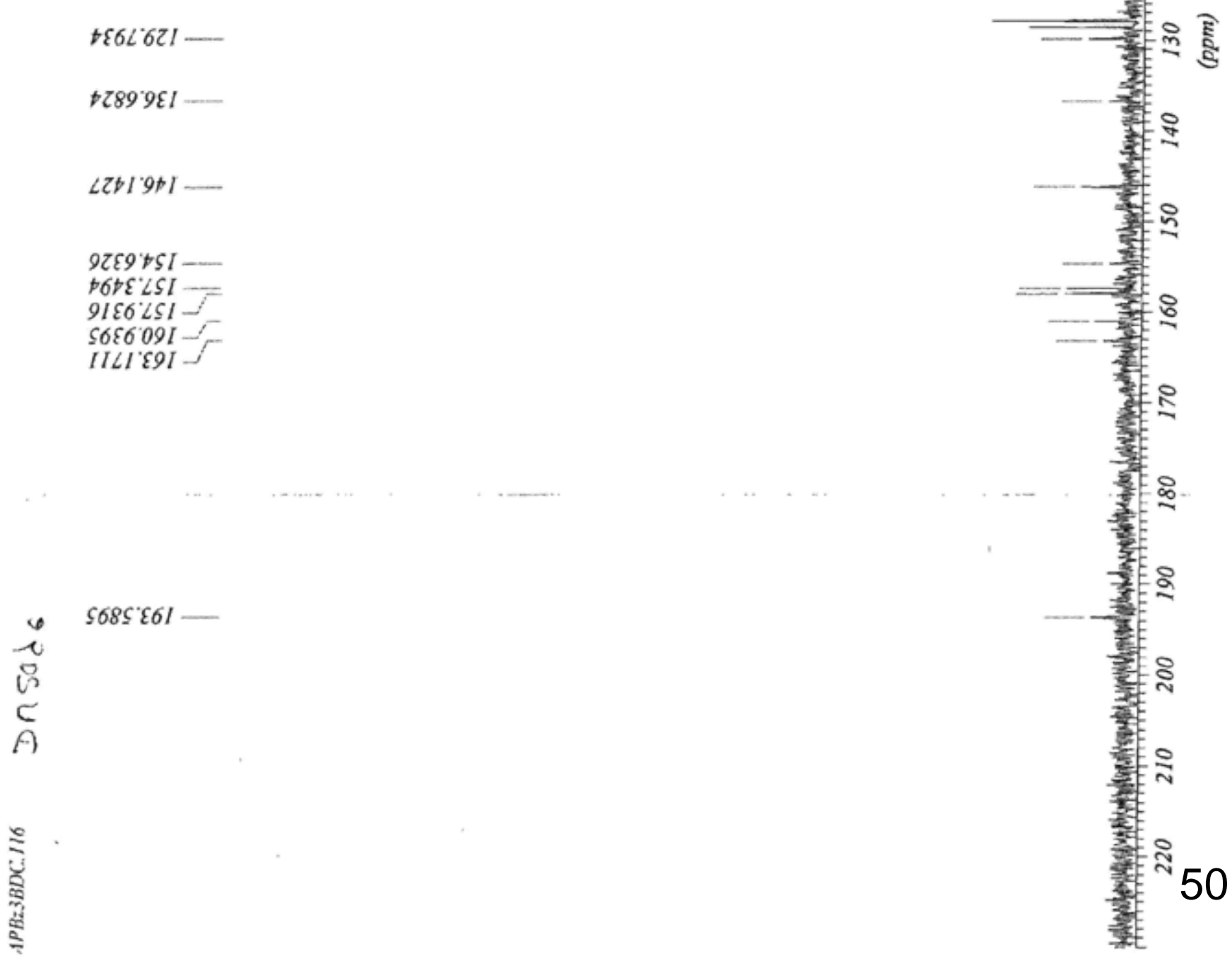
$\varepsilon 809^{\prime \prime}$

$99+9^{\circ} 1-$

$60 \angle 9^{\circ} 1-$

- $1989^{\circ} 1$

OZLLI

ZtZL'L

$0169 \mathrm{Z}$

$90 \varepsilon L Z$

ZL $92 Z 2$

11582

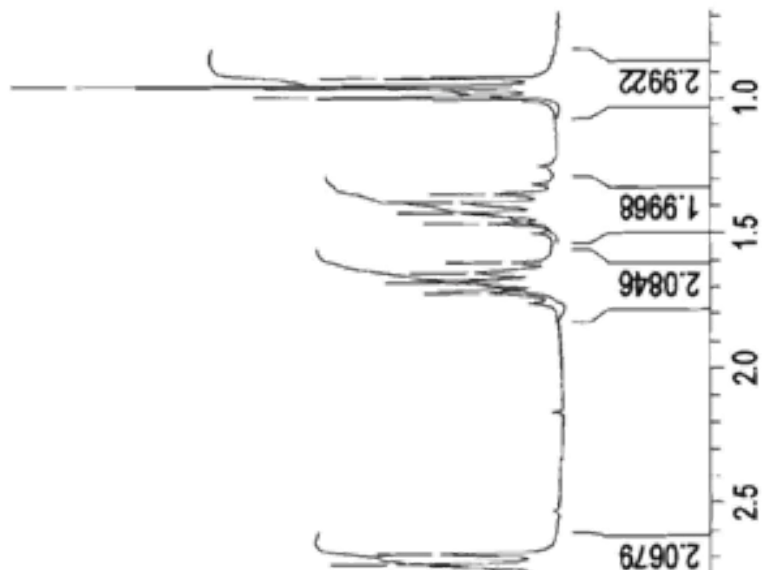

$4_{n B u}$

Olg2 $L$ OLSE'L

$\angle E 6 \varepsilon^{\prime} L$

60ES' $L-F$

EZSS' $L$

IZLS'L

SE6S'L -

259Z 8

t6928-

$6 t 678$

99088

6tBE 8

9L2t 8

1tSS 8

ES69'8

CL68'8

$66 £ 68$

6 เSเ 6
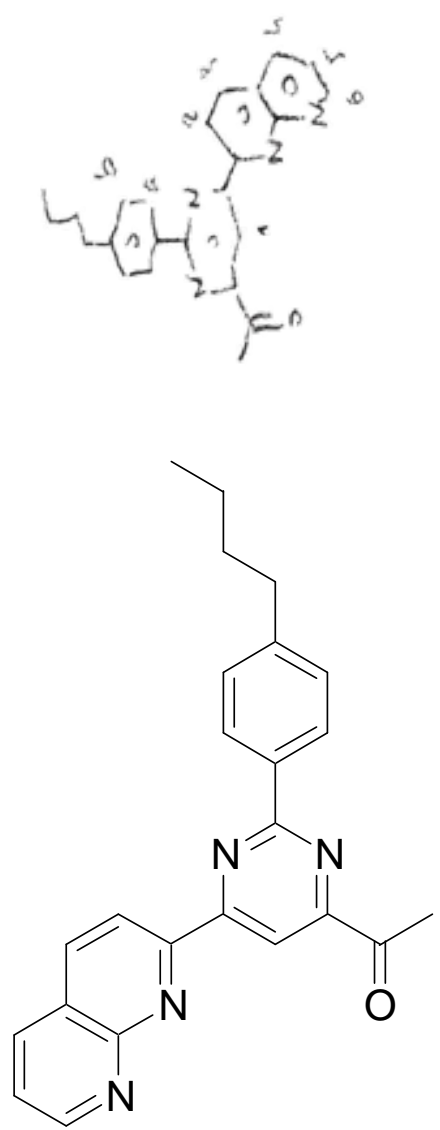

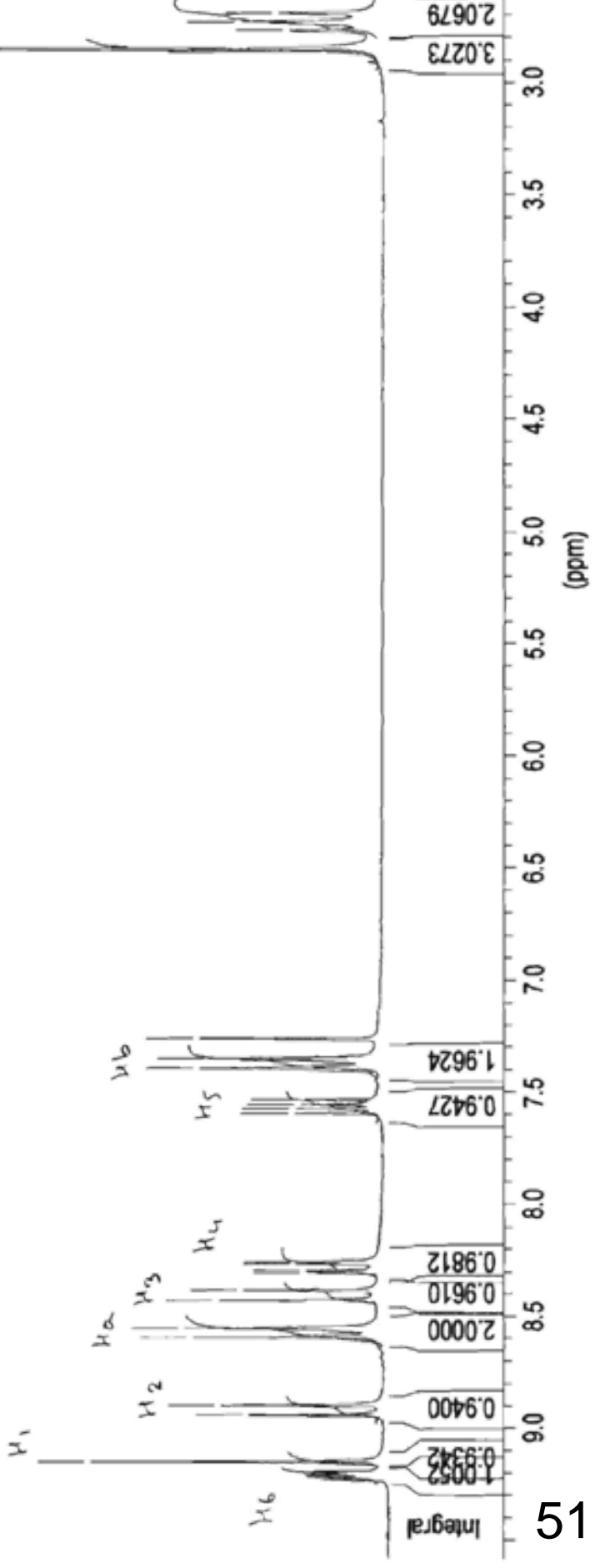


89t0"t -

6etorz-

๕ZL6'SZ -

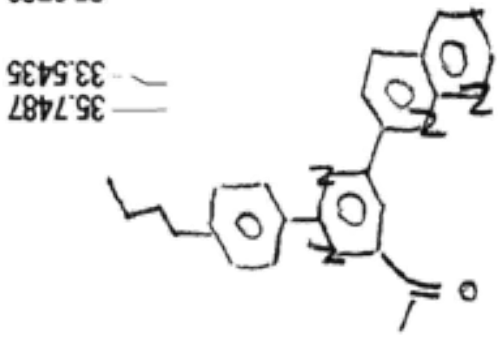

ن

⿶

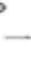

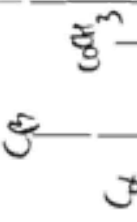

S68เ $O Z$ L

Z9S0'ZZ

เSL6 EZ!

เท!G 82 !

$89 \& 6^{\circ} 8 \mathrm{ZL}$

$8965 t \varepsilon l$

E6SO"LEL .

SLET BEL

$8 \varepsilon t<9 t$

$\varepsilon 08 \nabla \forall S$

8128 '

L $\angle E ' \angle S$.

90ع6 $09 \mathrm{~L}$.

0199't9

$\forall 928$ เ
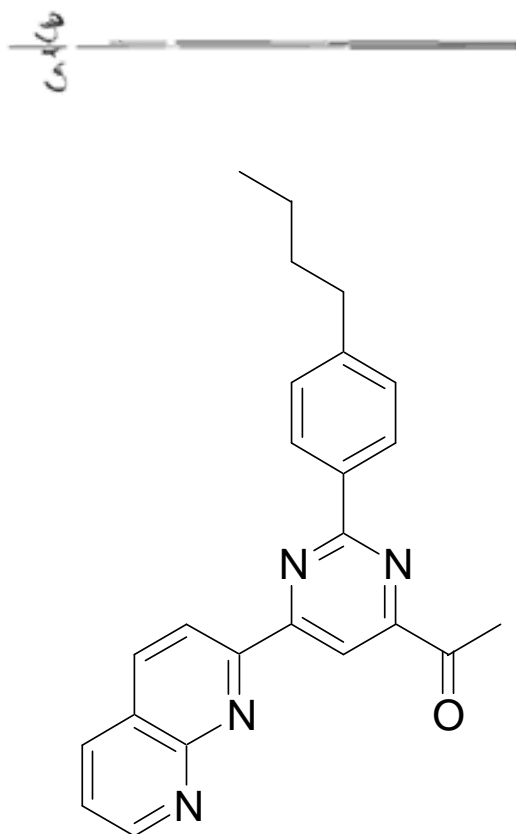


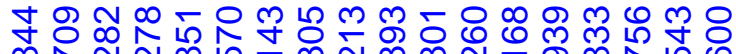

$\infty N$ N $\infty$ म

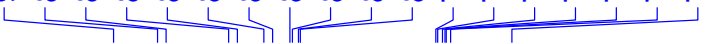

$\mathbf{4}_{\text {tertBu }}$
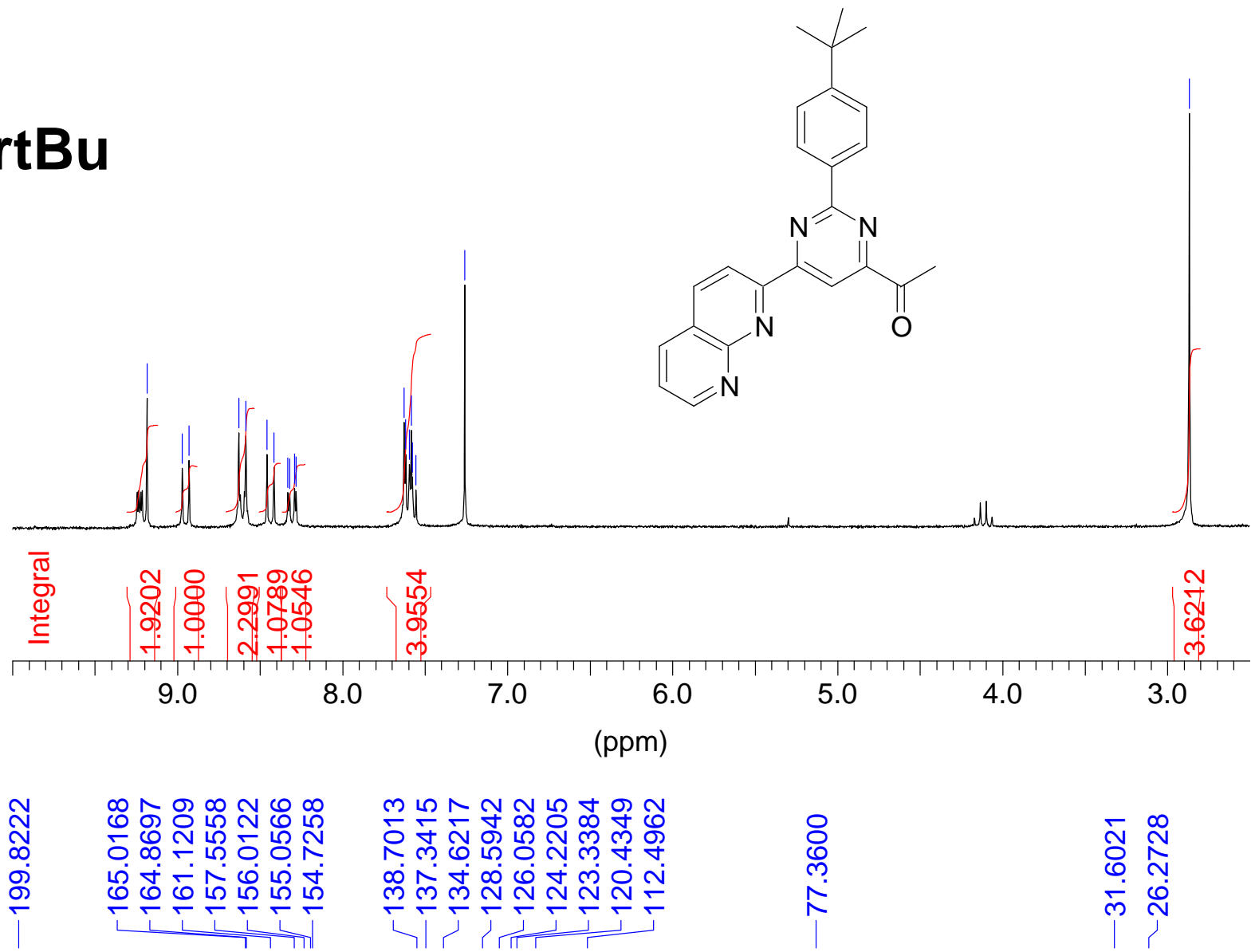

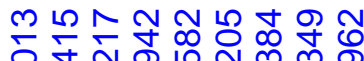
กิ

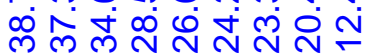

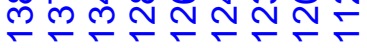<smiles>CC(=O)c1cc(-c2ccc3cccnc3n2)nc(-c2ccc(C(C)(C)C)cc2)n1</smiles>
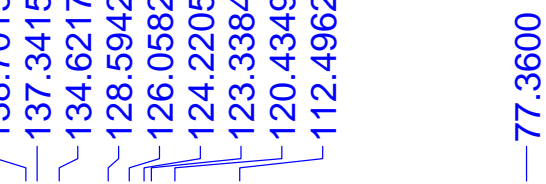

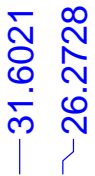

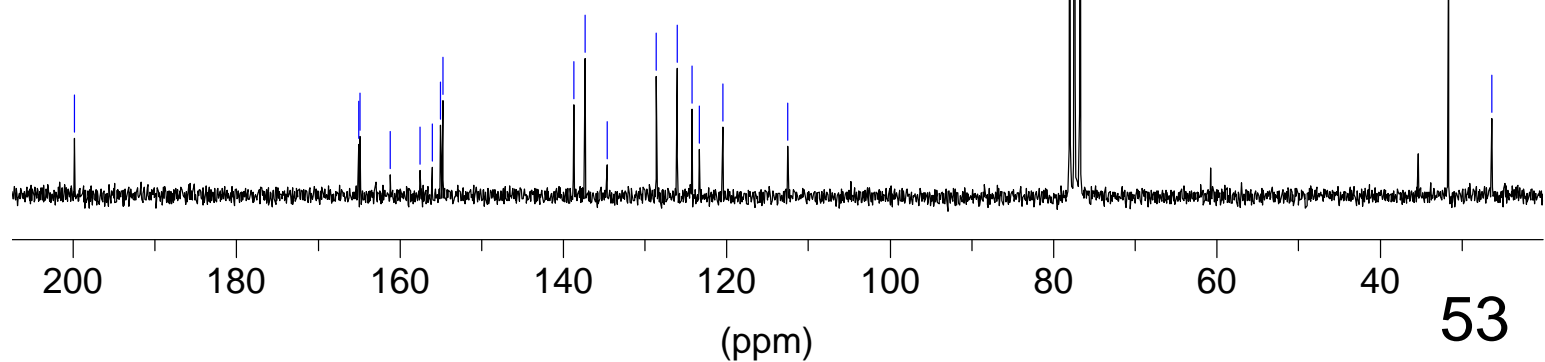




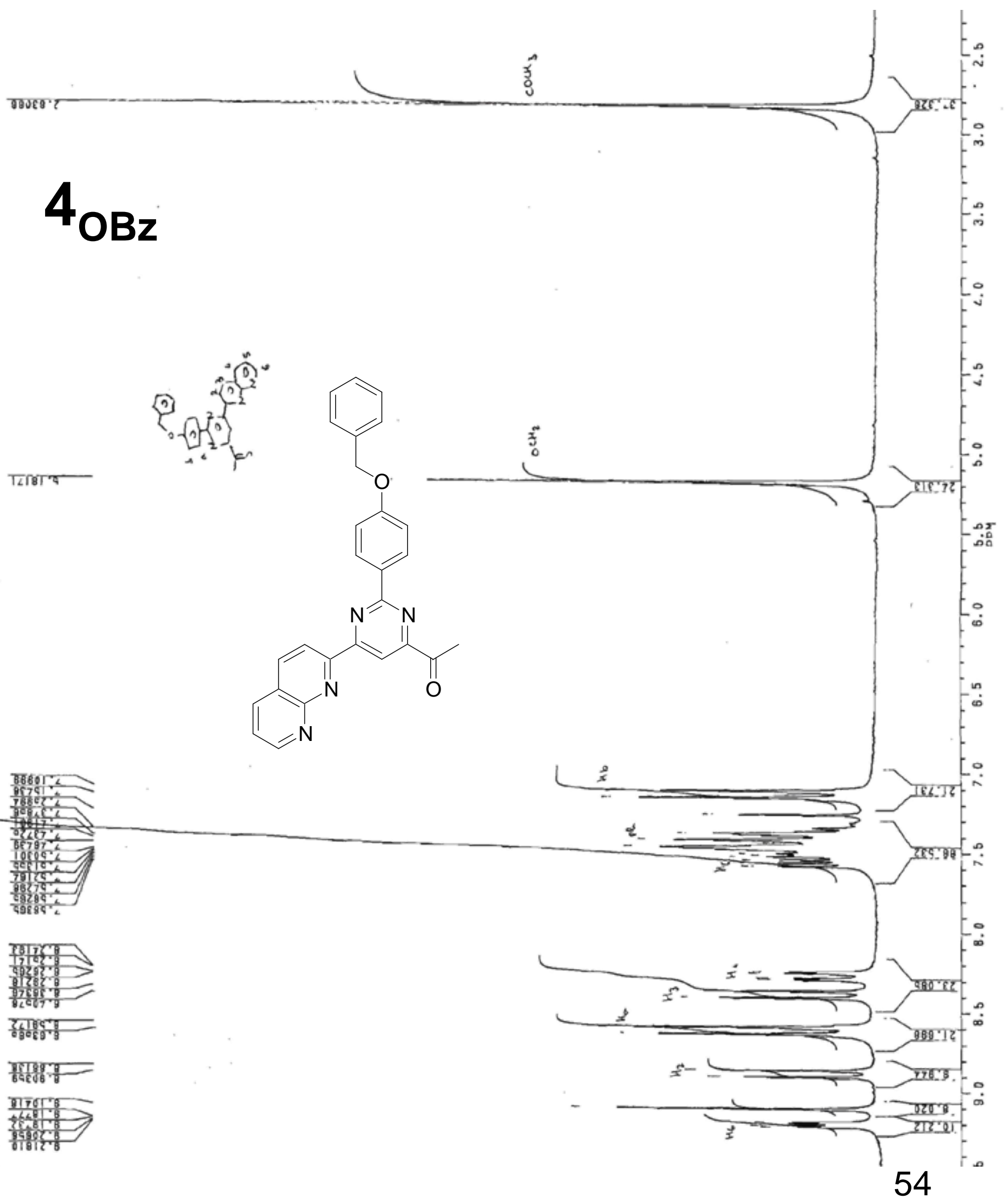




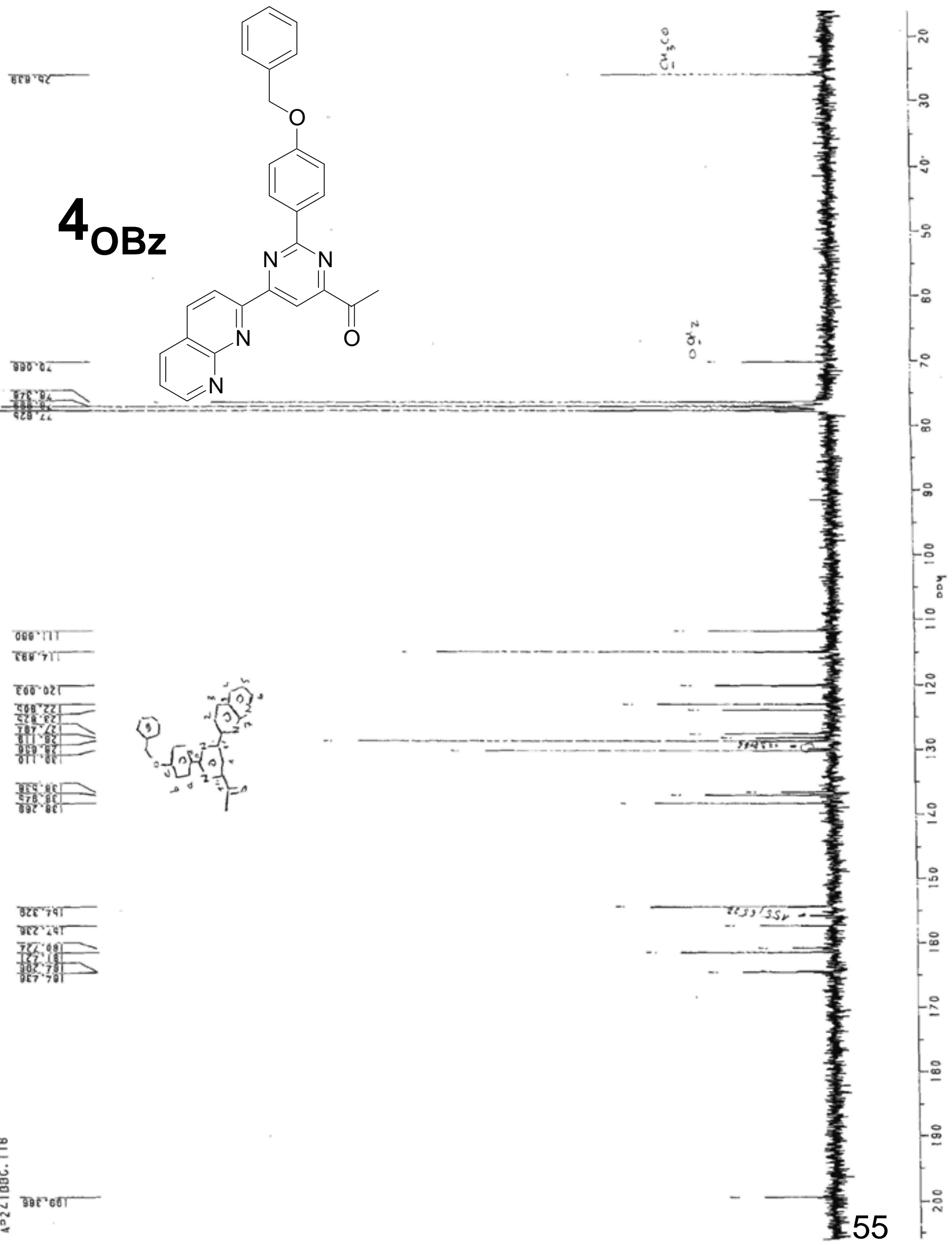




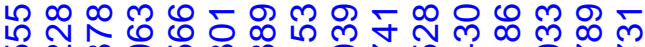

ᄂ $N \infty$ \%

$\infty \infty^{\infty} \infty \infty^{\infty} \infty \infty^{\infty}$

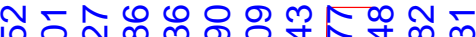
व 0 m

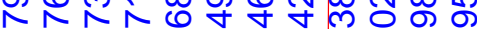
nัn n

\section{$5_{(\mathrm{nBu})(\mathrm{nBu})}$}
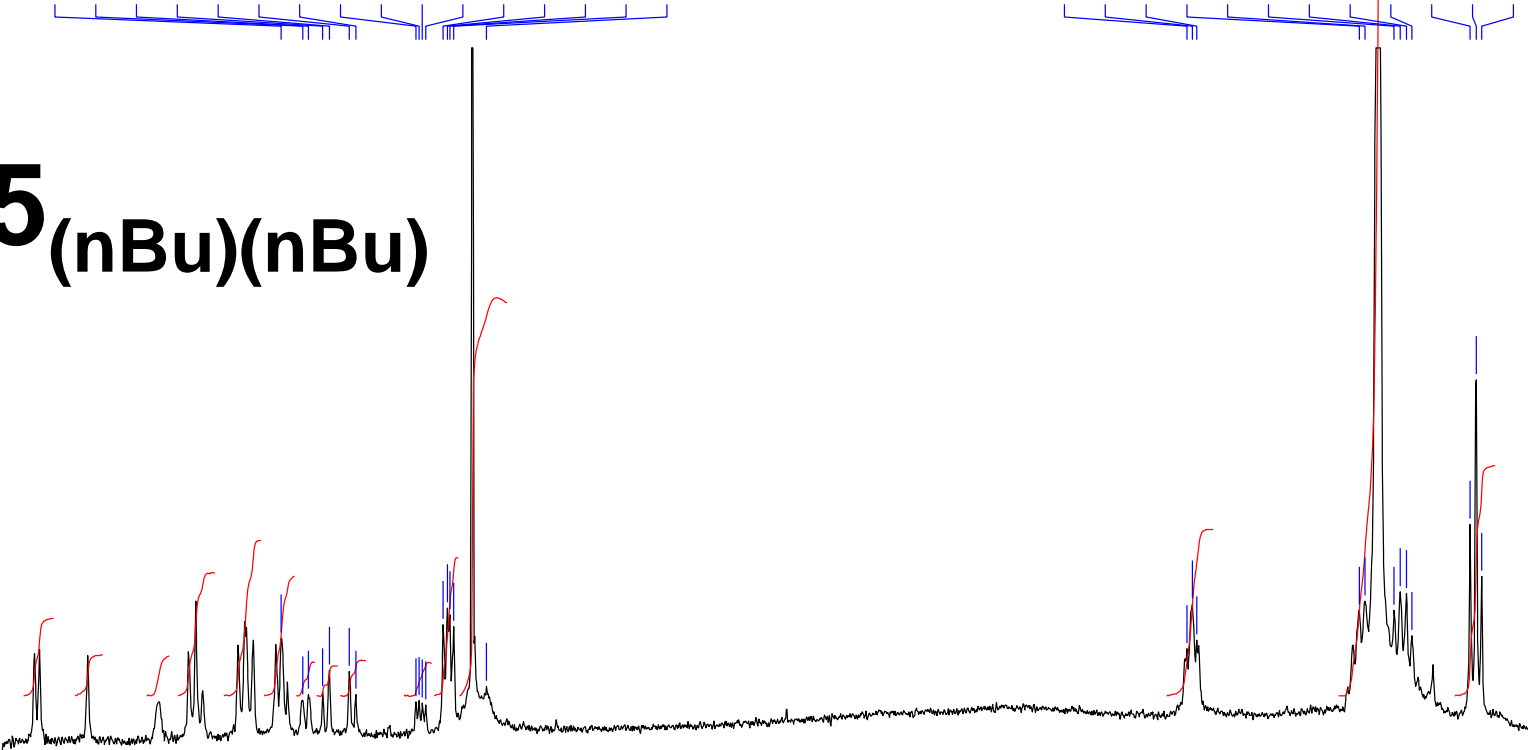

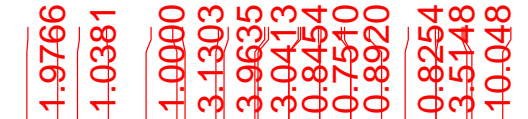

10
9

8
7
6
ป

3

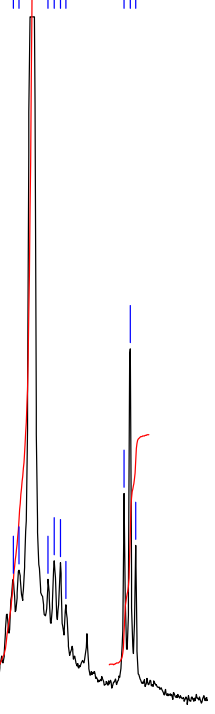

4

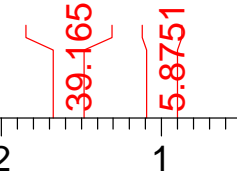

(ppm)

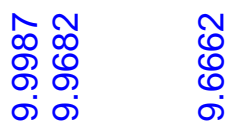

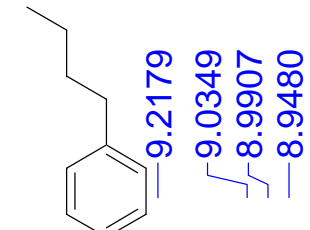

オ্̛ำ N a

ᄂ

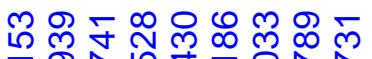

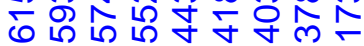
隹

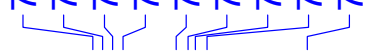<smiles>CCCCc1ccc(-c2nc(-c3ccc4cccnc4n3)cc(-c3ccc4ccc(-c5cc(-c6ccc(C=O)c(N)n6)nc(C)n5)nc4n3)n2)cc1</smiles>

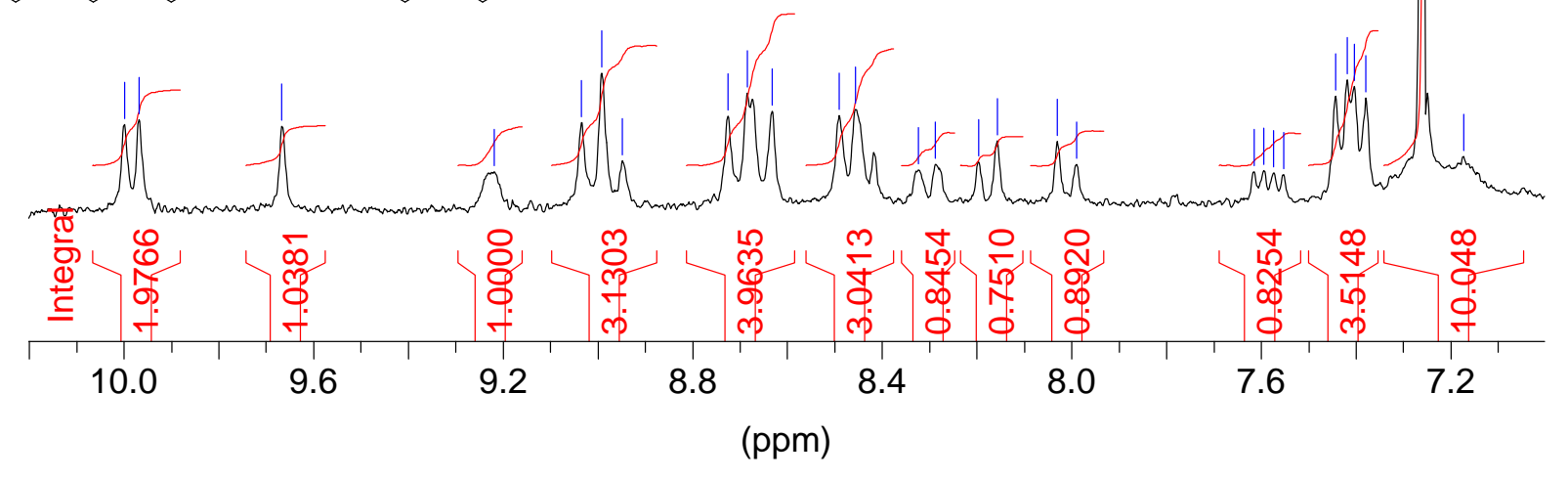




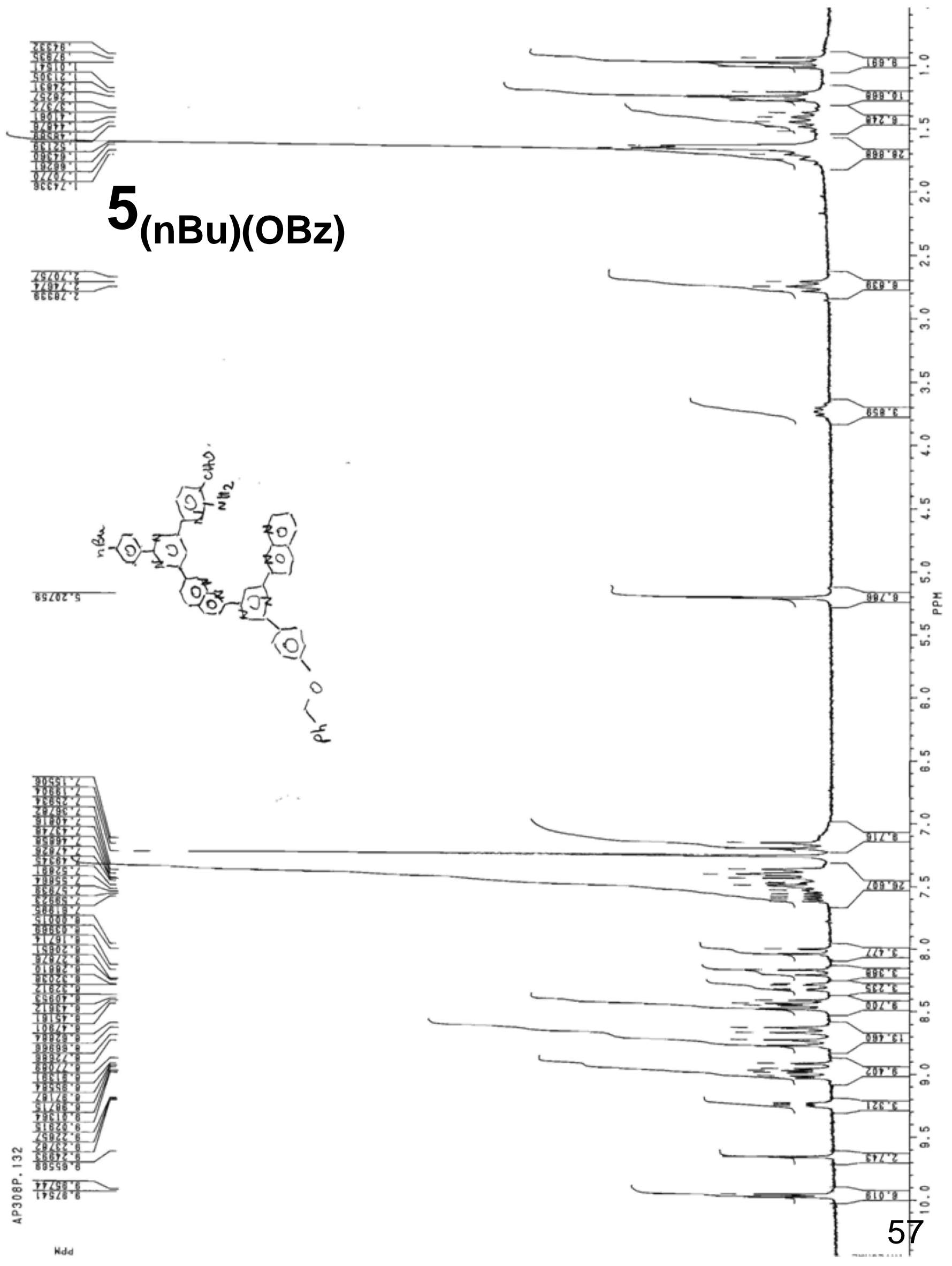


$6_{\mathrm{nBu}}$
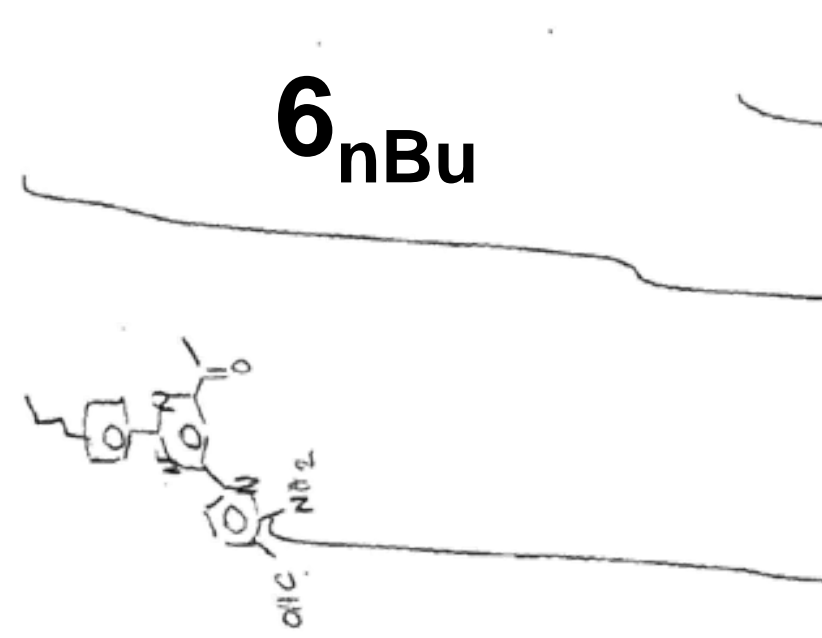
$-\boldsymbol{6}_{\mathrm{nBu}}$

वहलेट

蓶照

18962

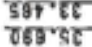

70.57 -
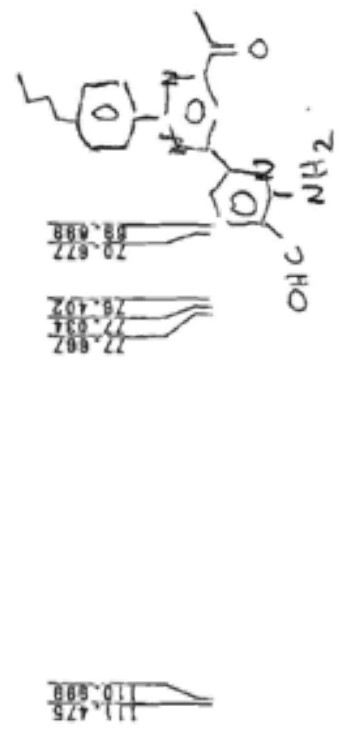

गणन्डा

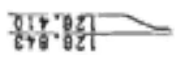

75\%

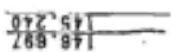

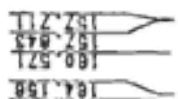

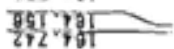

$\operatorname{TaE} \gamma_{B}$

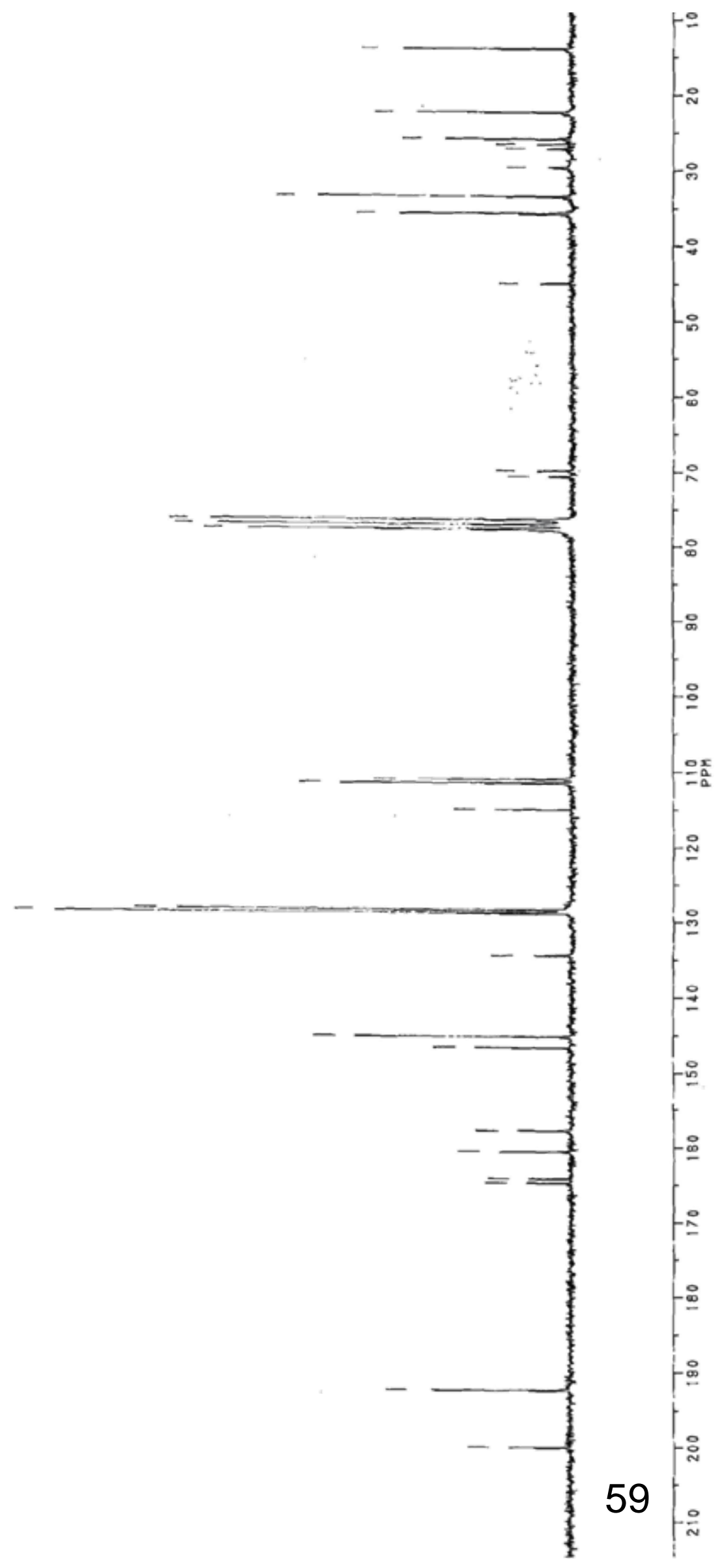



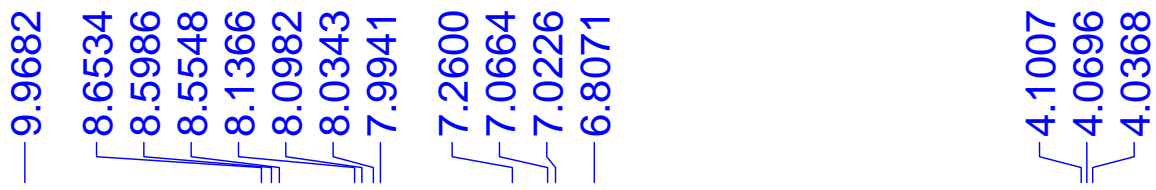

の

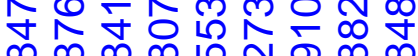

$6_{\mathrm{OC} 12}$

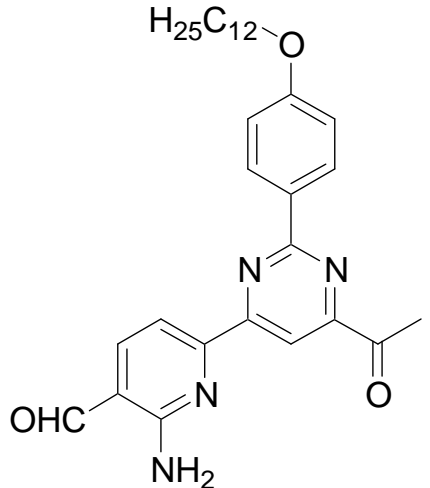

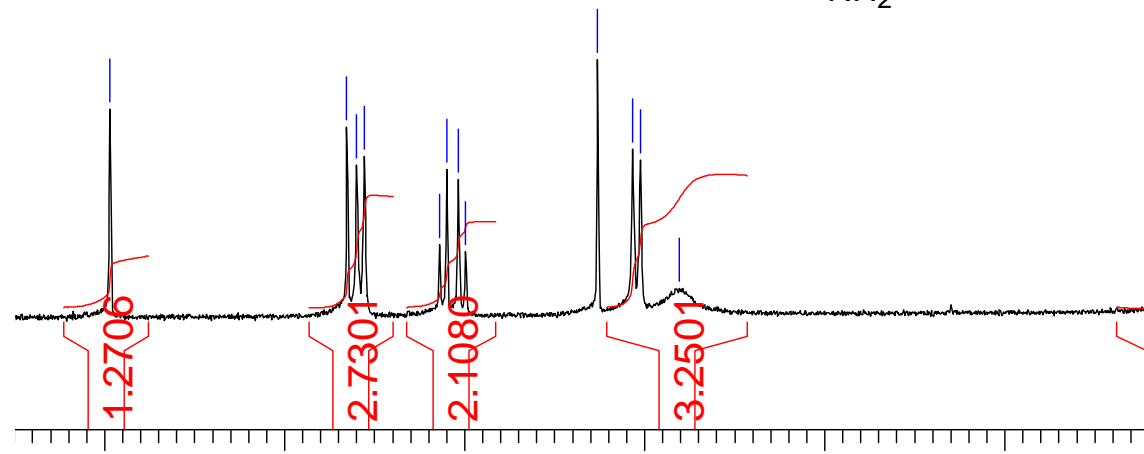

10

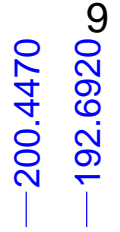

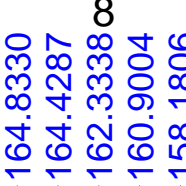

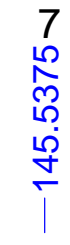

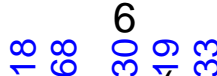

5

4

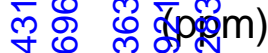

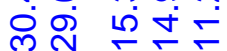

규 각각

๑

N

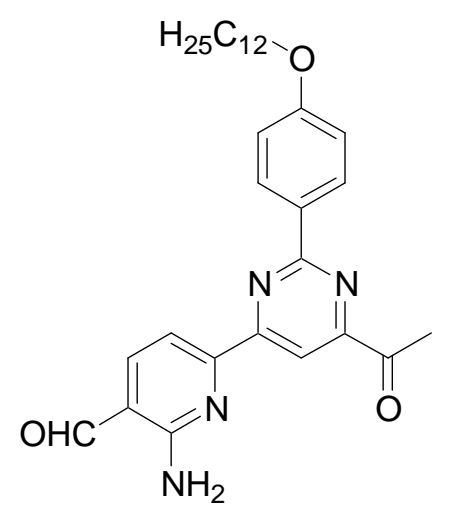

त.

N $\quad$ ஸे

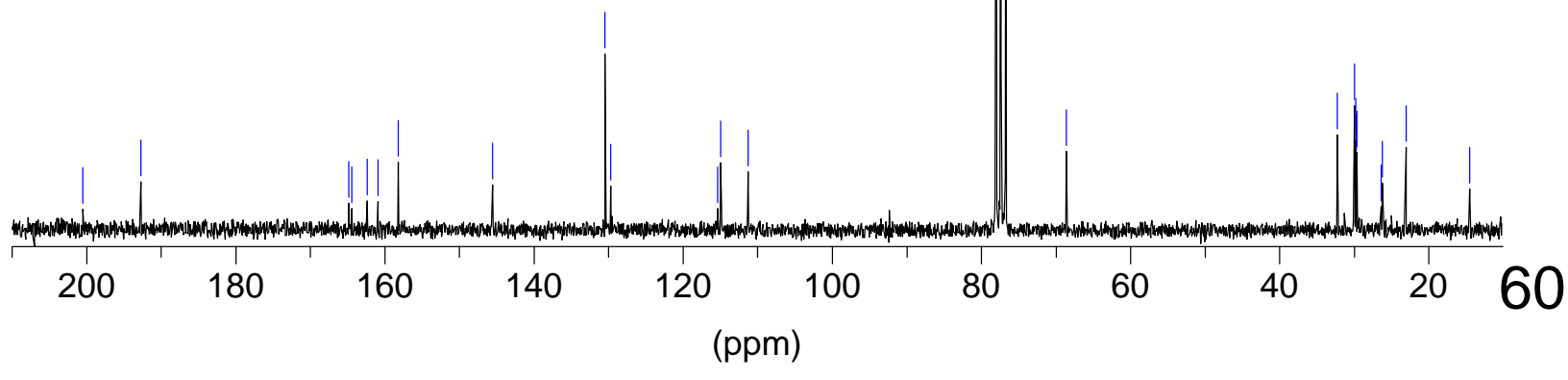




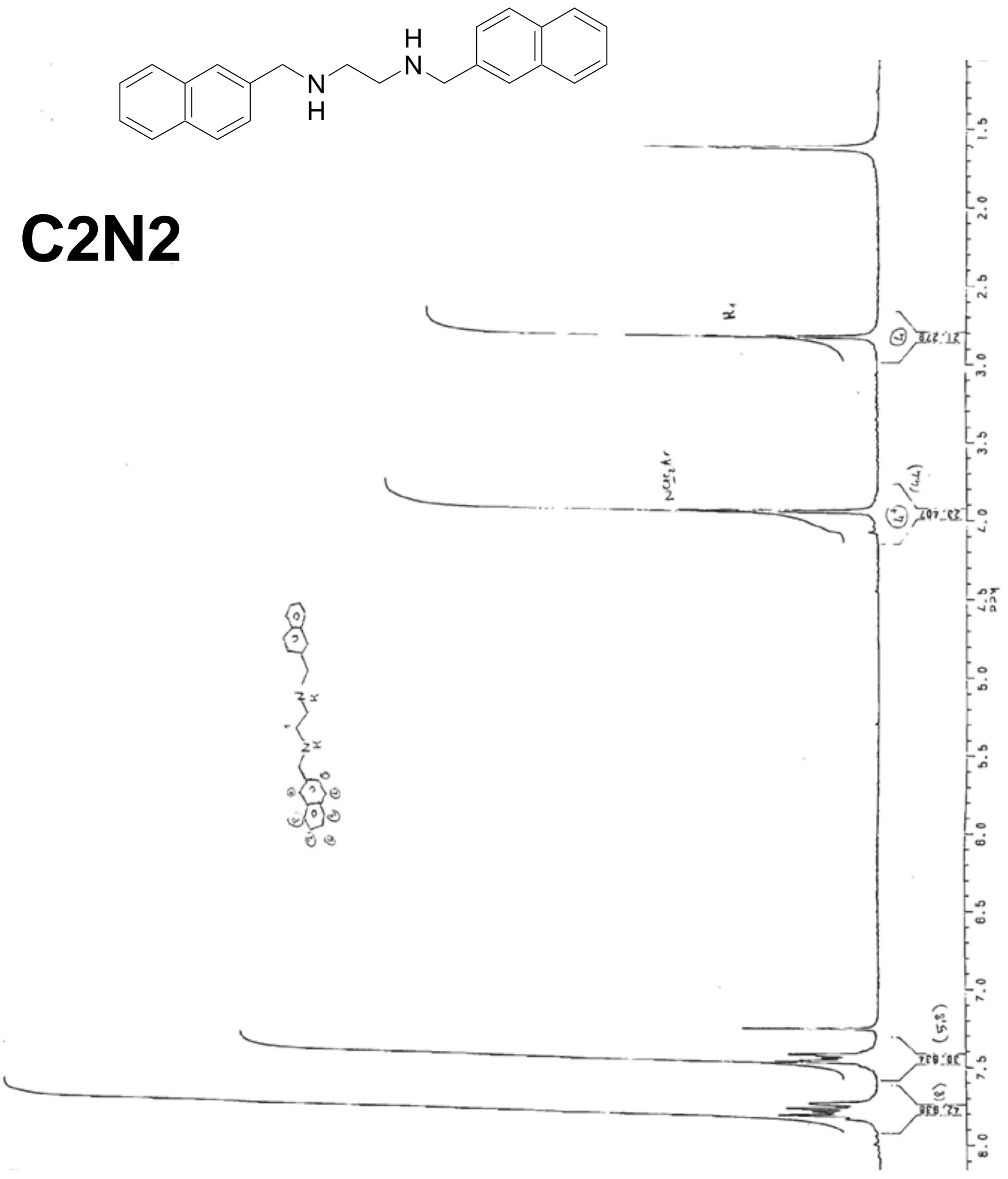

61 


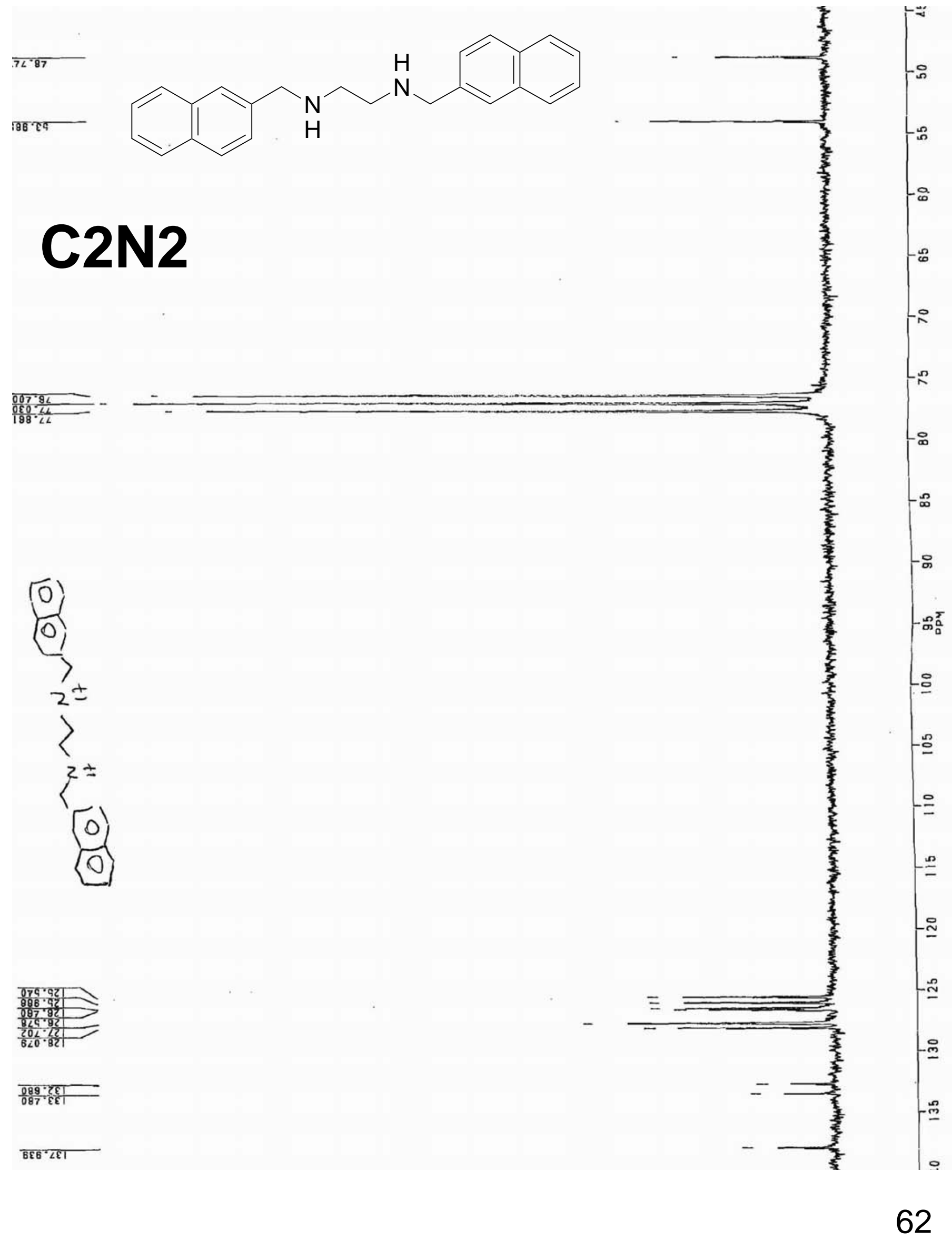




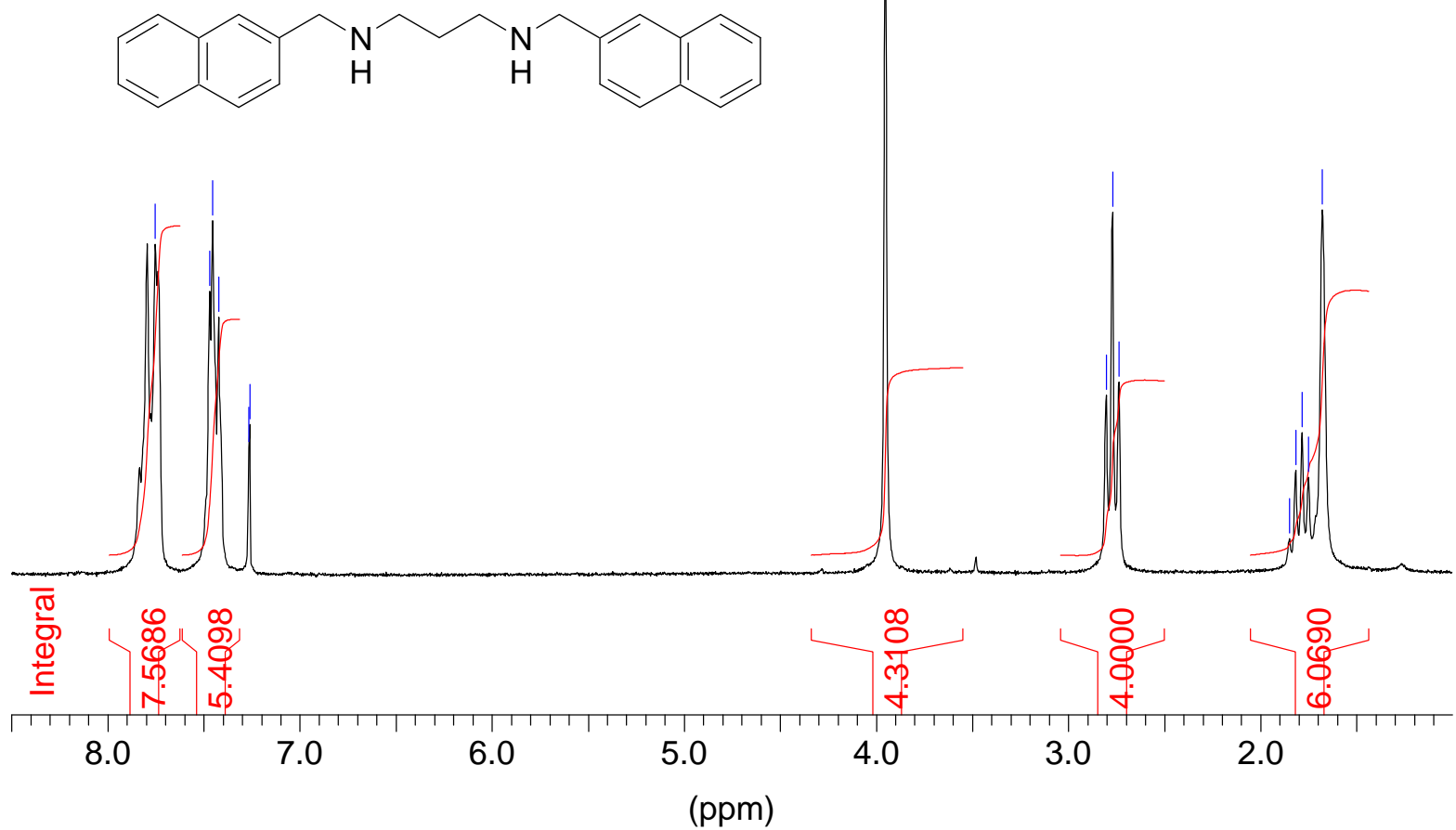

승 용 লे $\infty$ लं

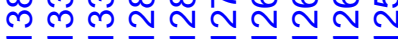<smiles>c1ccc2cc(CNCCCNCc3ccc4ccccc4c3)ccc2c1</smiles>

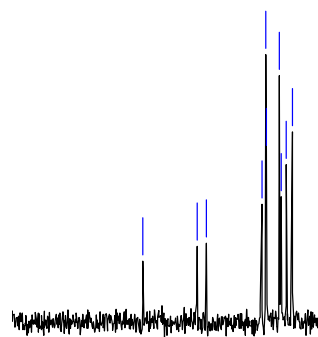


ำ

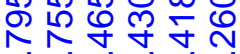

ベNヘN<smiles>c1ccc2cc(CNCCNCCNCc3ccc4ccccc4c3)ccc2c1</smiles>
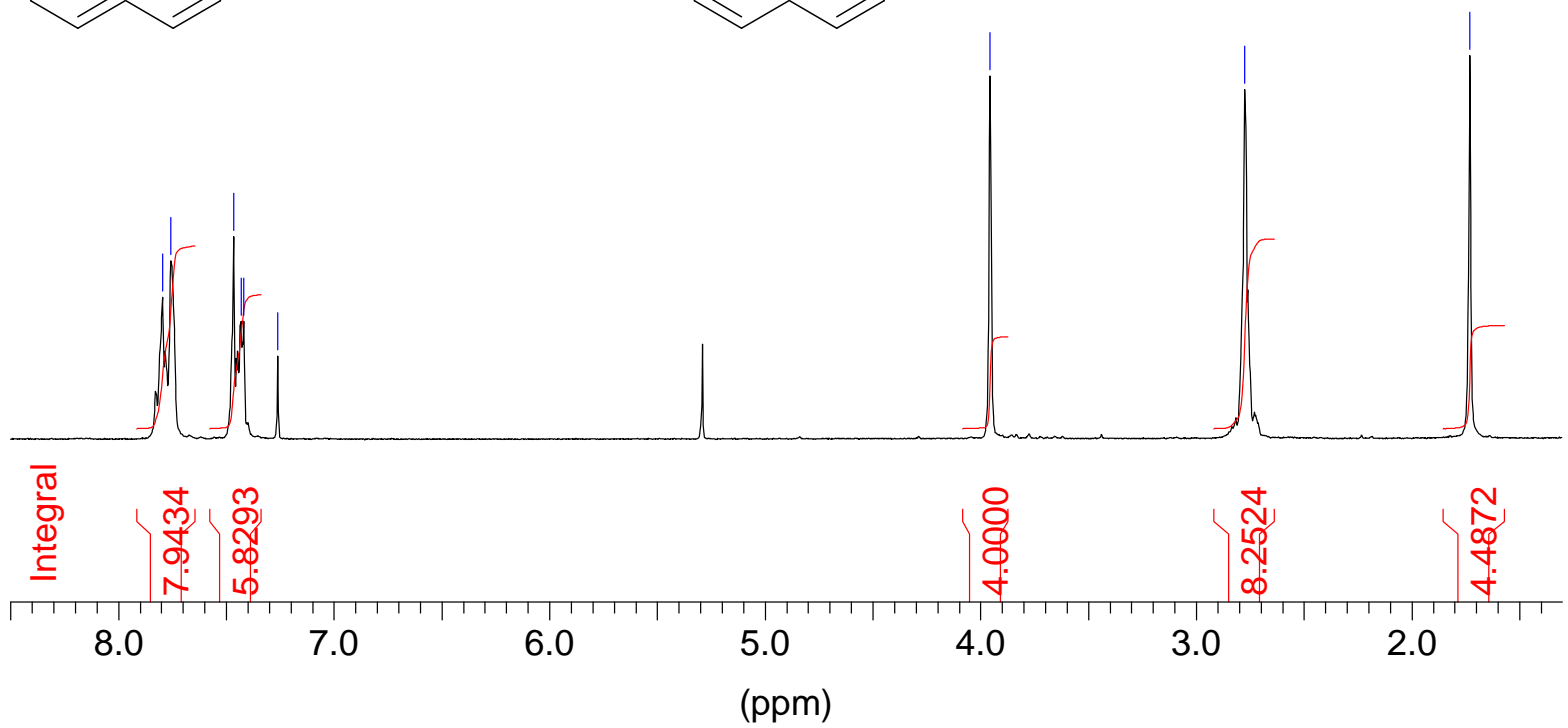

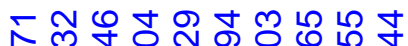

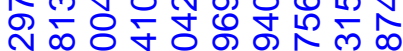

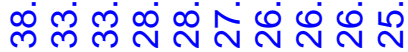

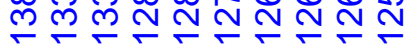<smiles>c1ccc2cc(CNCCNCCNCc3ccc4ccccc4c3)ccc2c1</smiles>

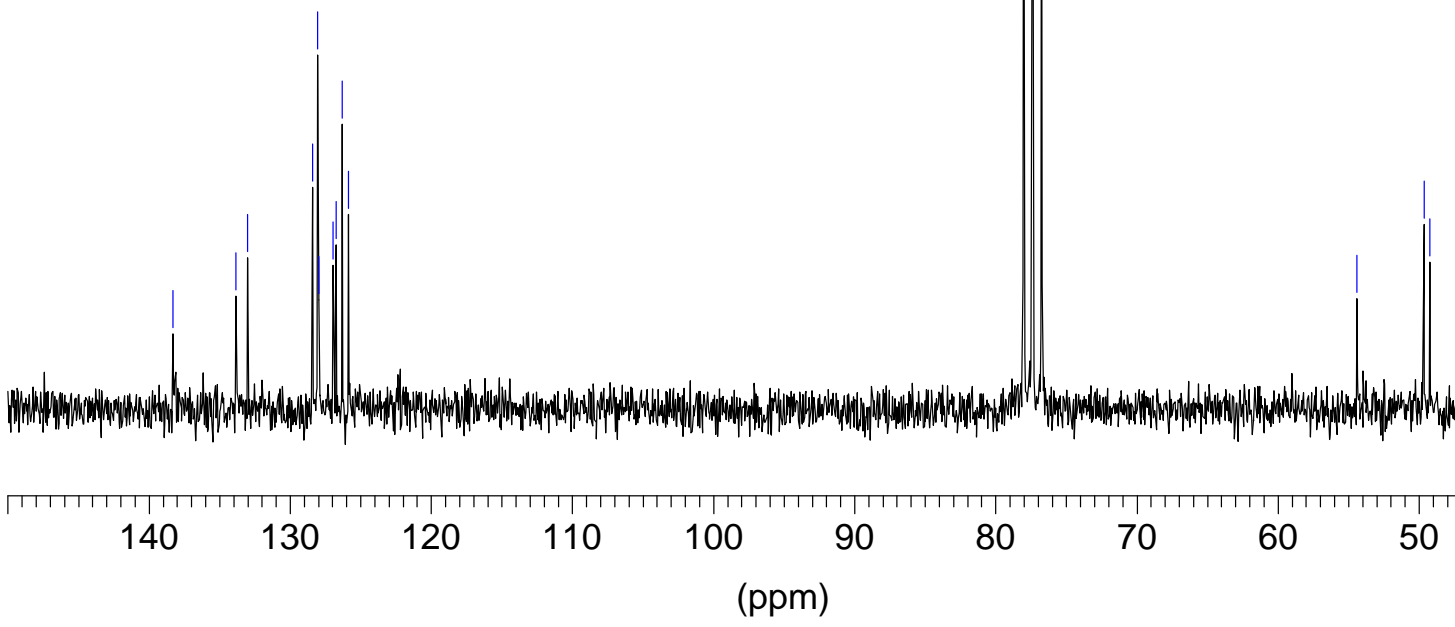


ำก

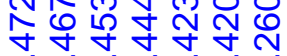

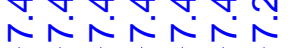

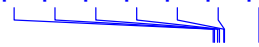

\section{C33N3}

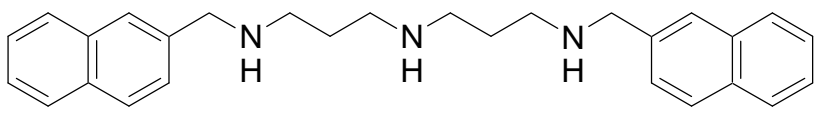
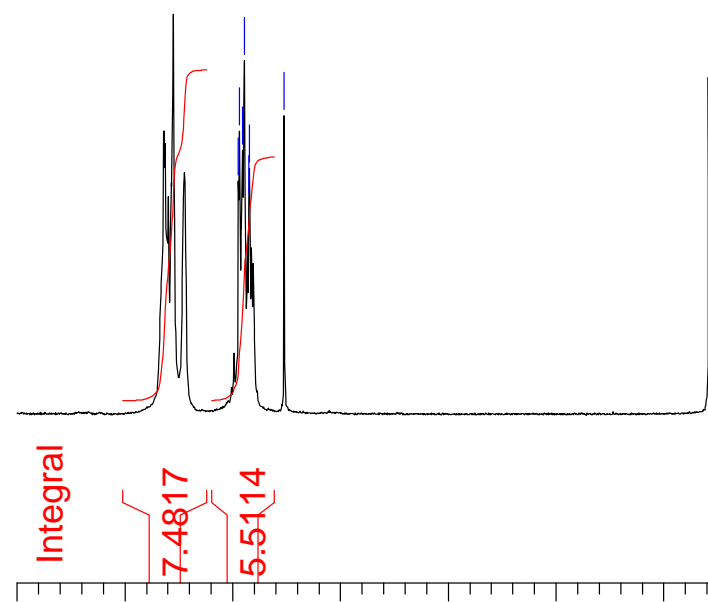

8.0
7.0

m ๙ m ल

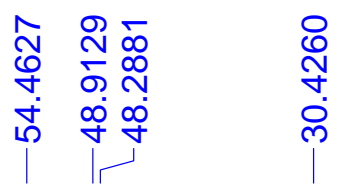

孛

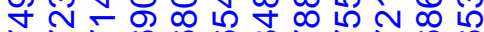
N

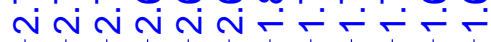

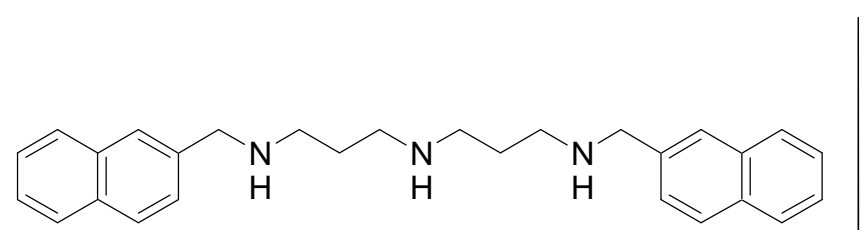

(ppm)

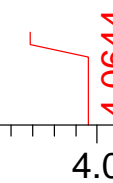

3.0
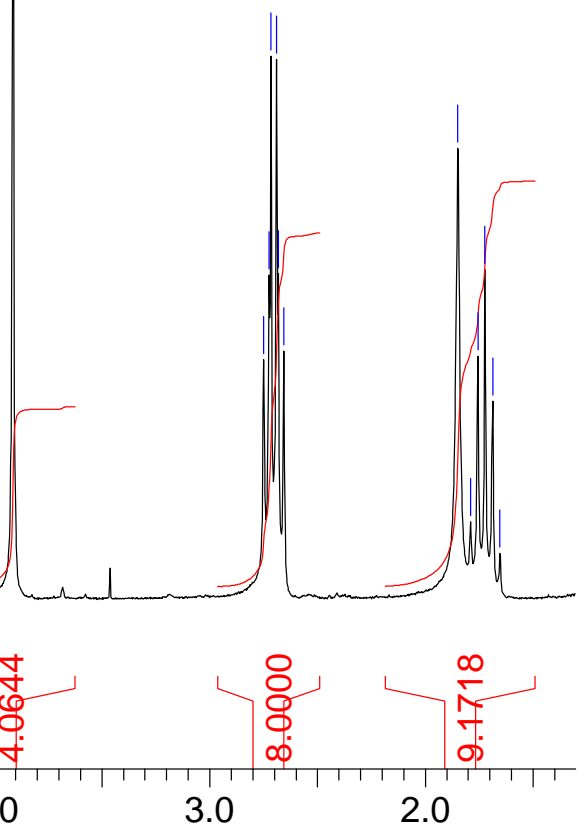


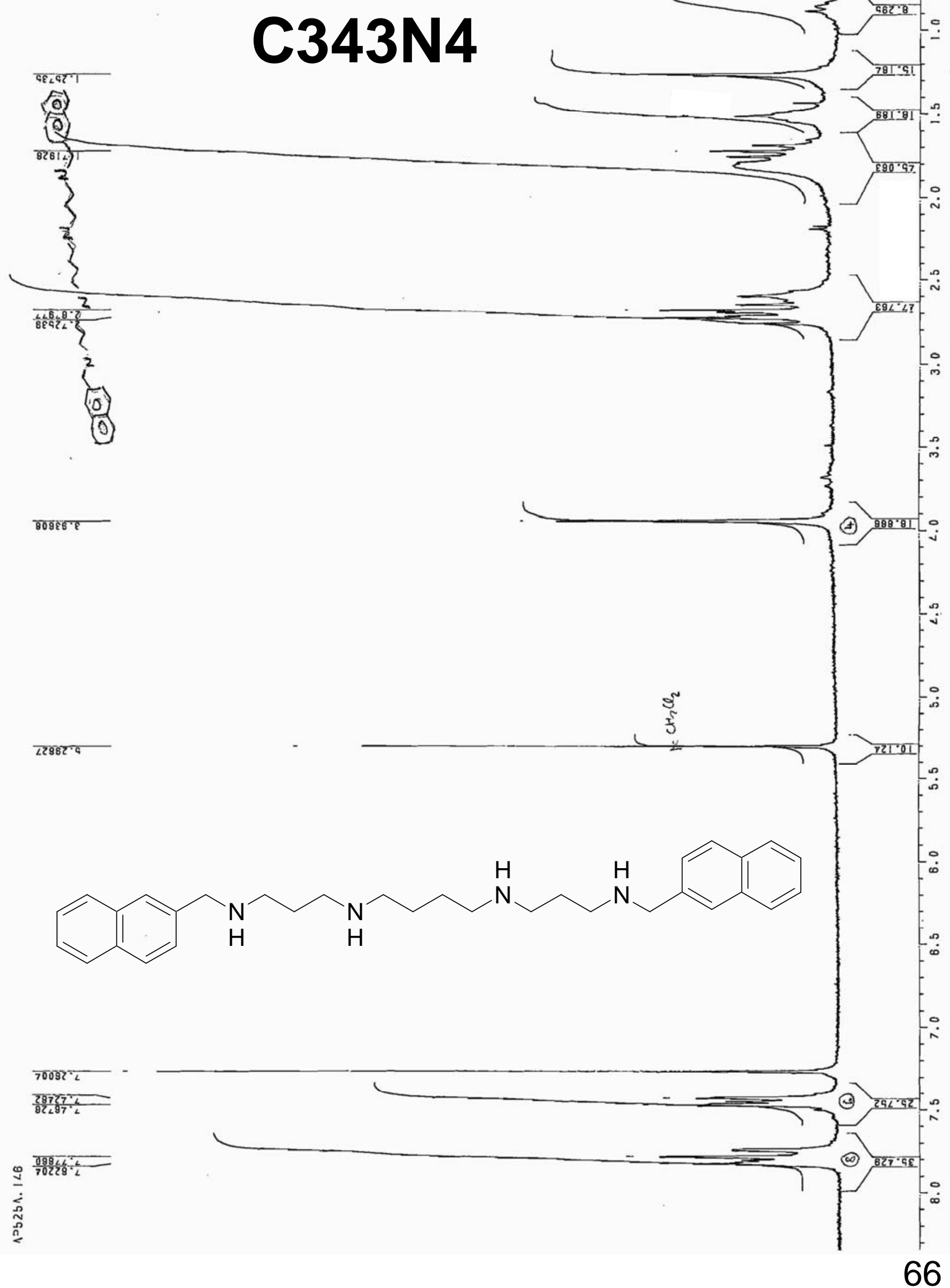



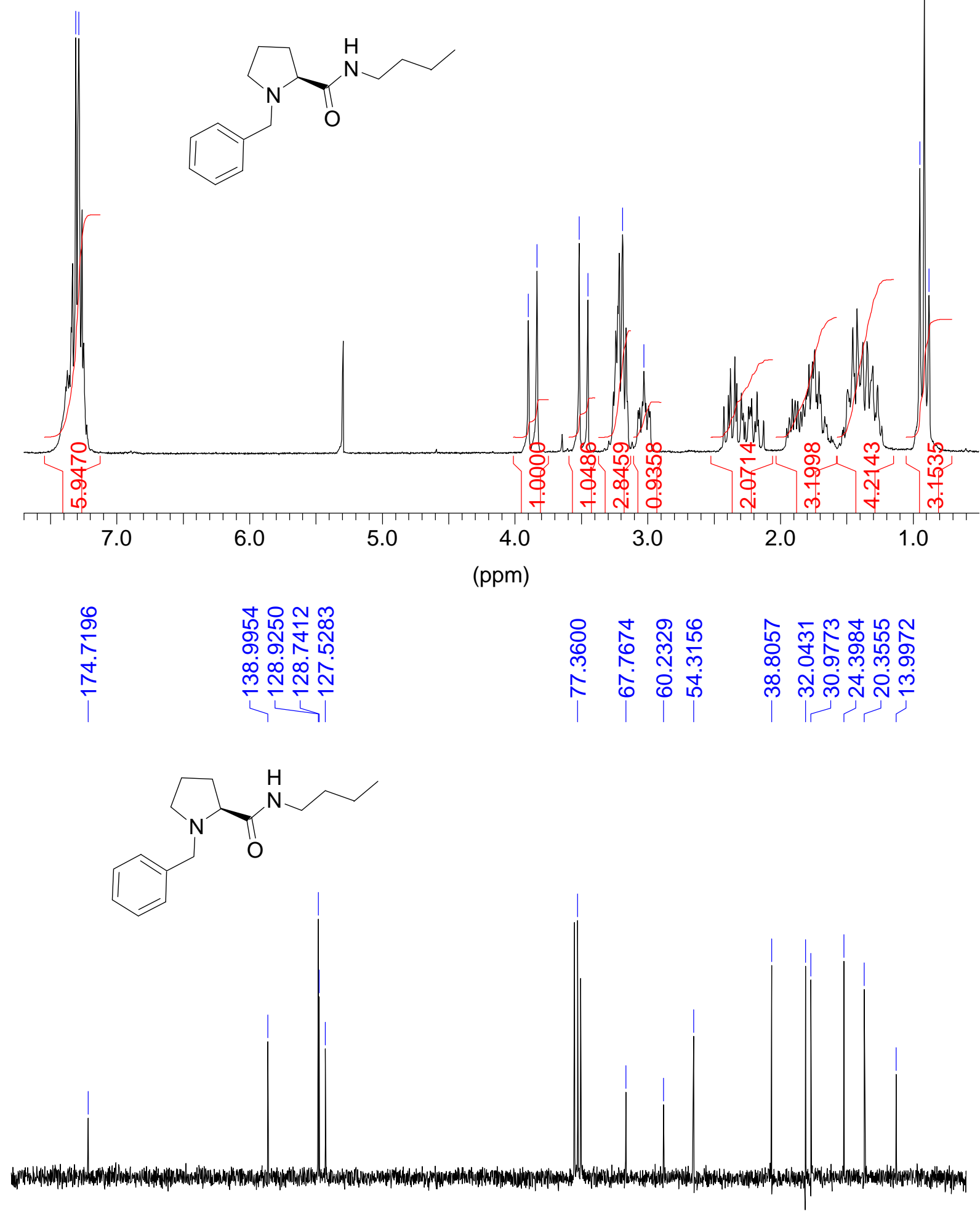

\begin{tabular}{|c|c|c|c|c|c|c|c|c|c|}
\hline 180 & 160 & 140 & 120 & 100 & 80 & 60 & 40 & 20 \\
$(\mathrm{ppm})$ & &
\end{tabular}



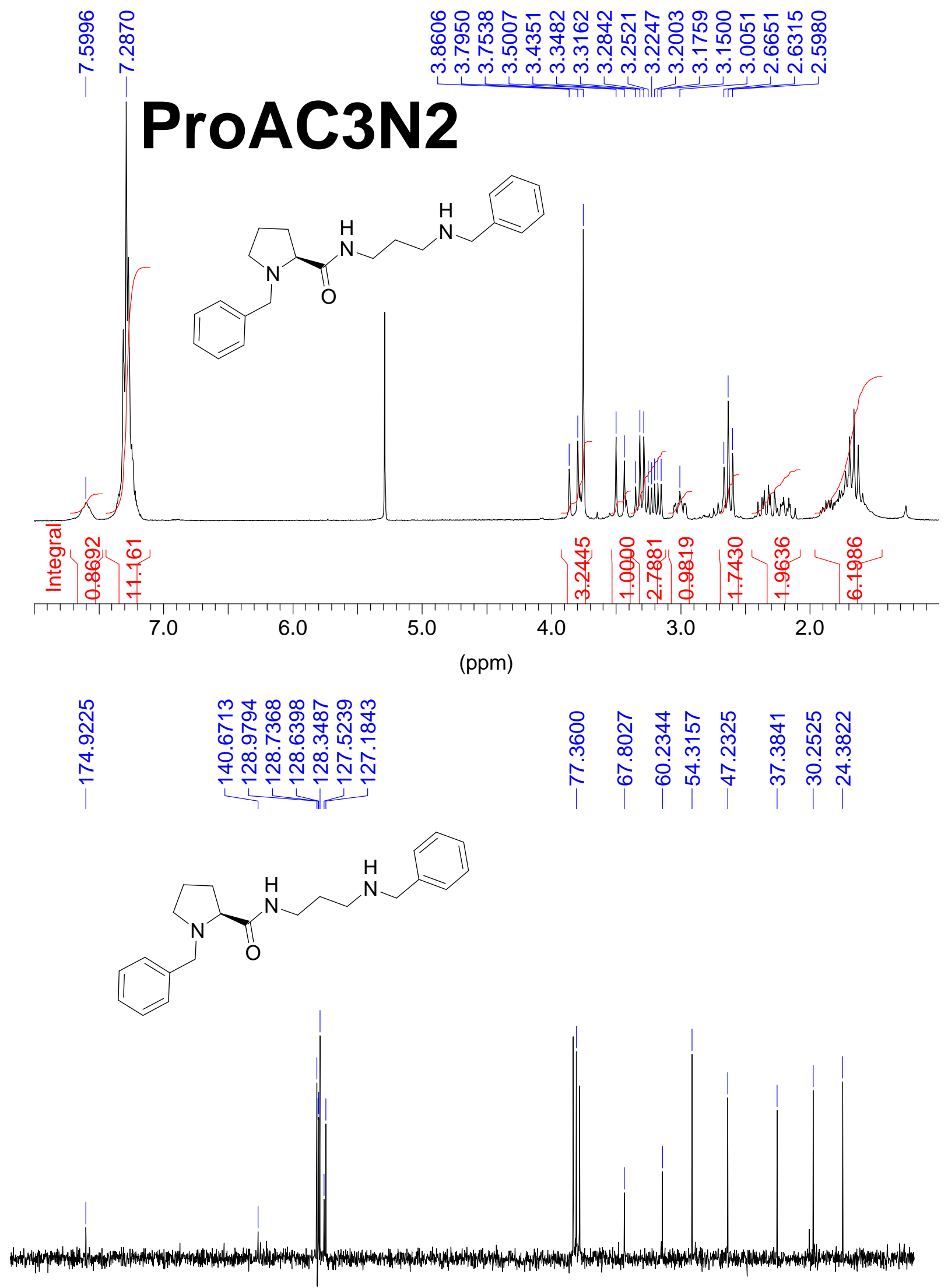

\begin{tabular}{|l|l|l|r|r|r|r|r|r|r|}
\hline 180 & 160 & 140 & 120 & 100 & 80 & 60 & 40 & 20 \\
$(\mathrm{ppm})$ & &
\end{tabular}



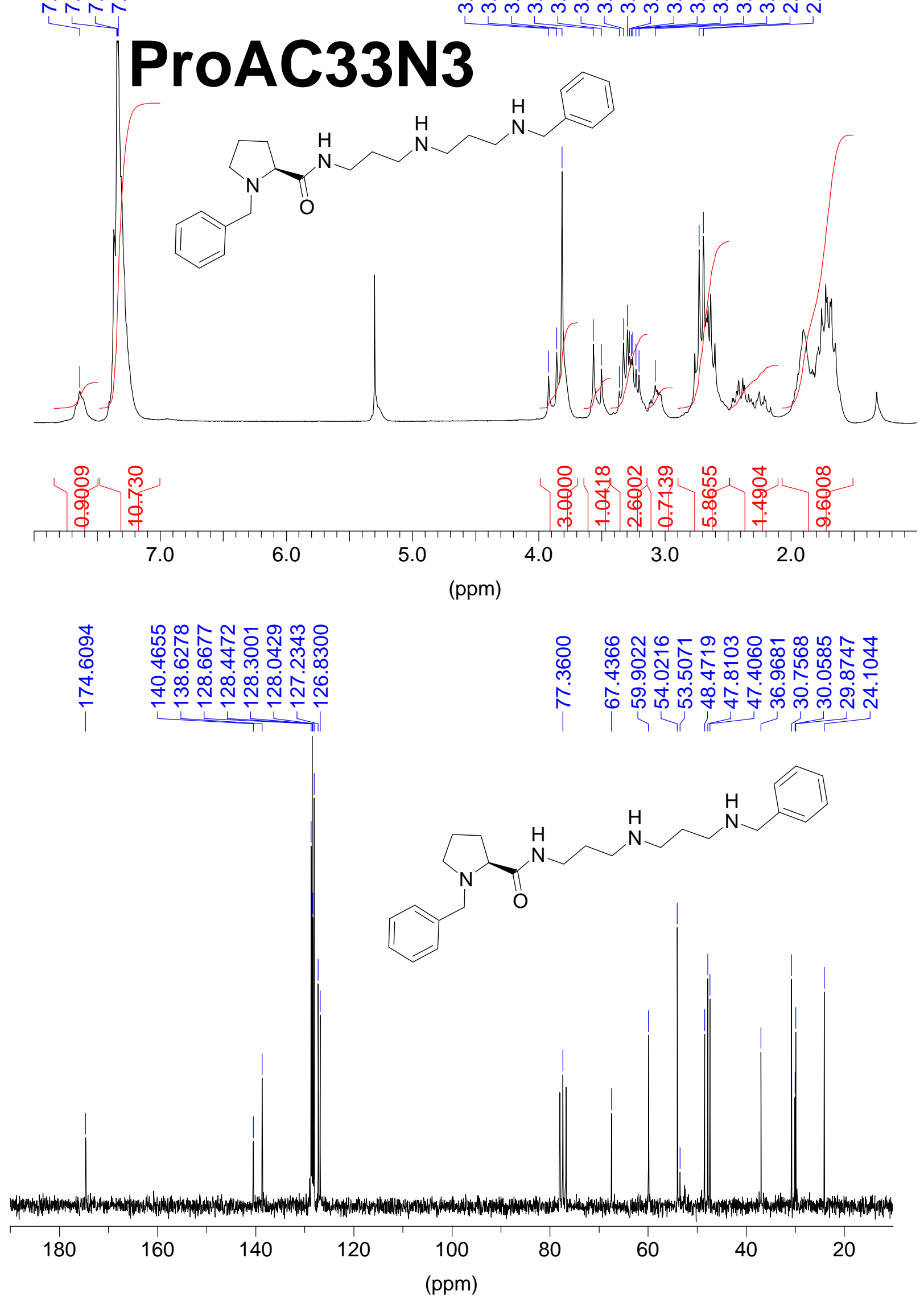
<smiles>NCCCNCCCNCc1ccccc1</smiles>

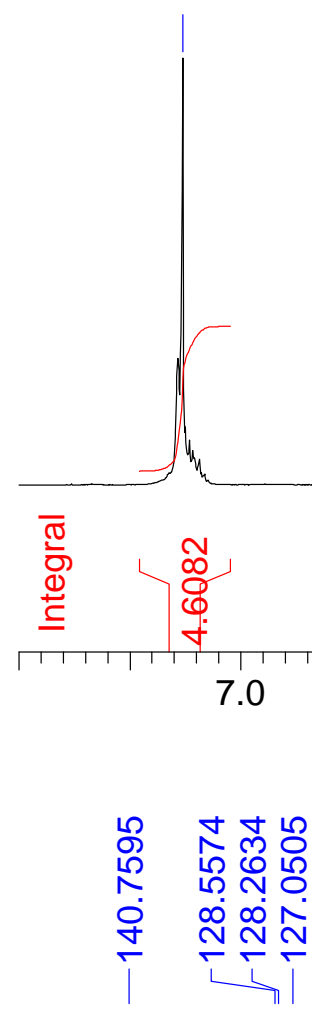<smiles>NCCCNCCCNCc1ccccc1</smiles>

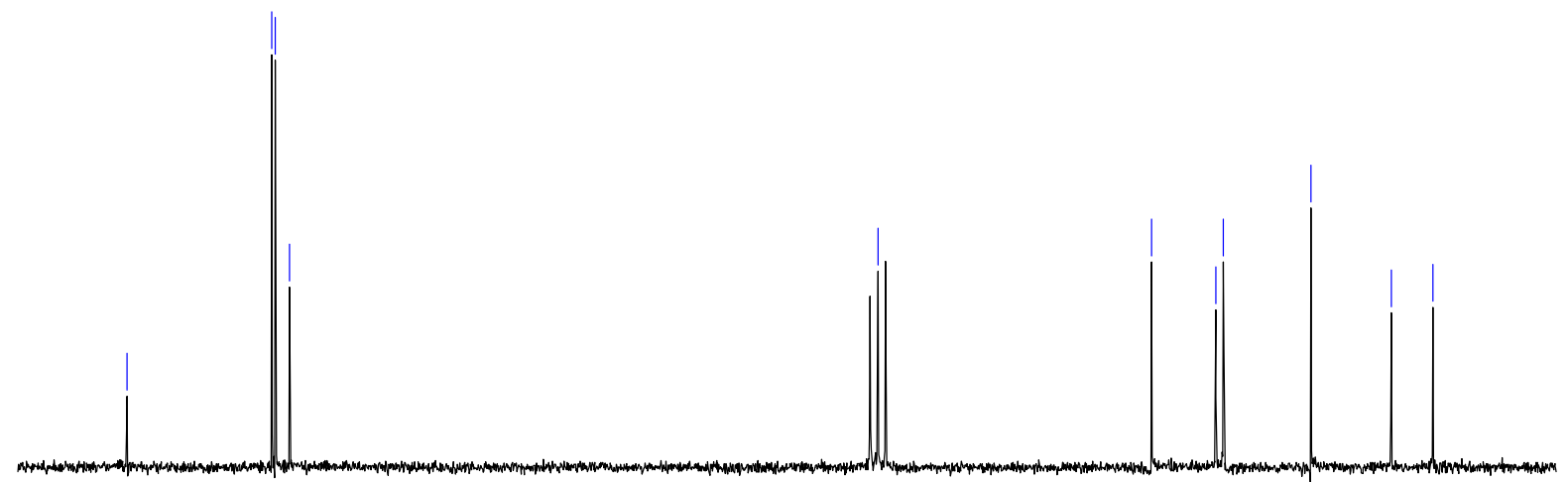



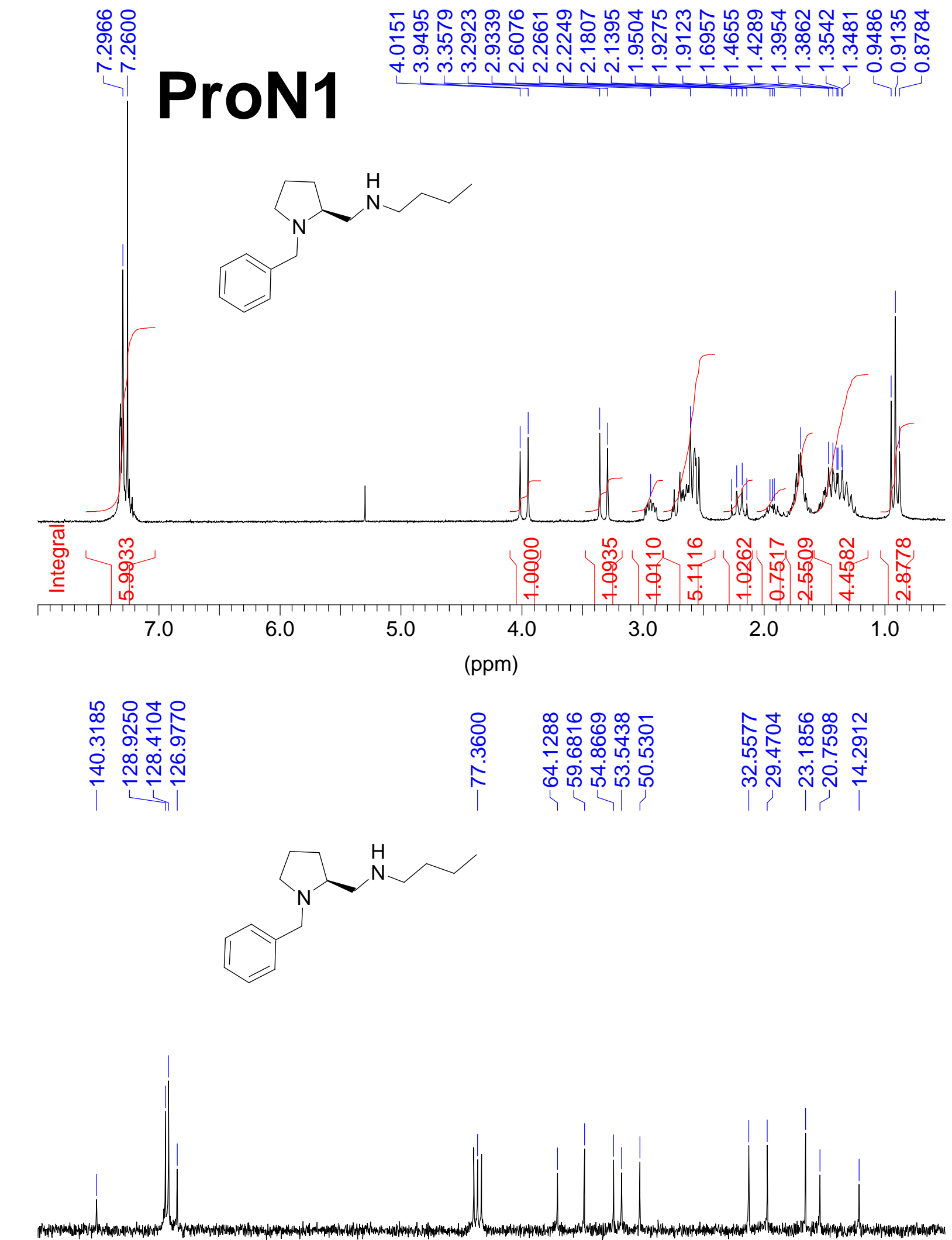

¿

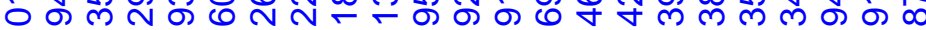
N ProN1 


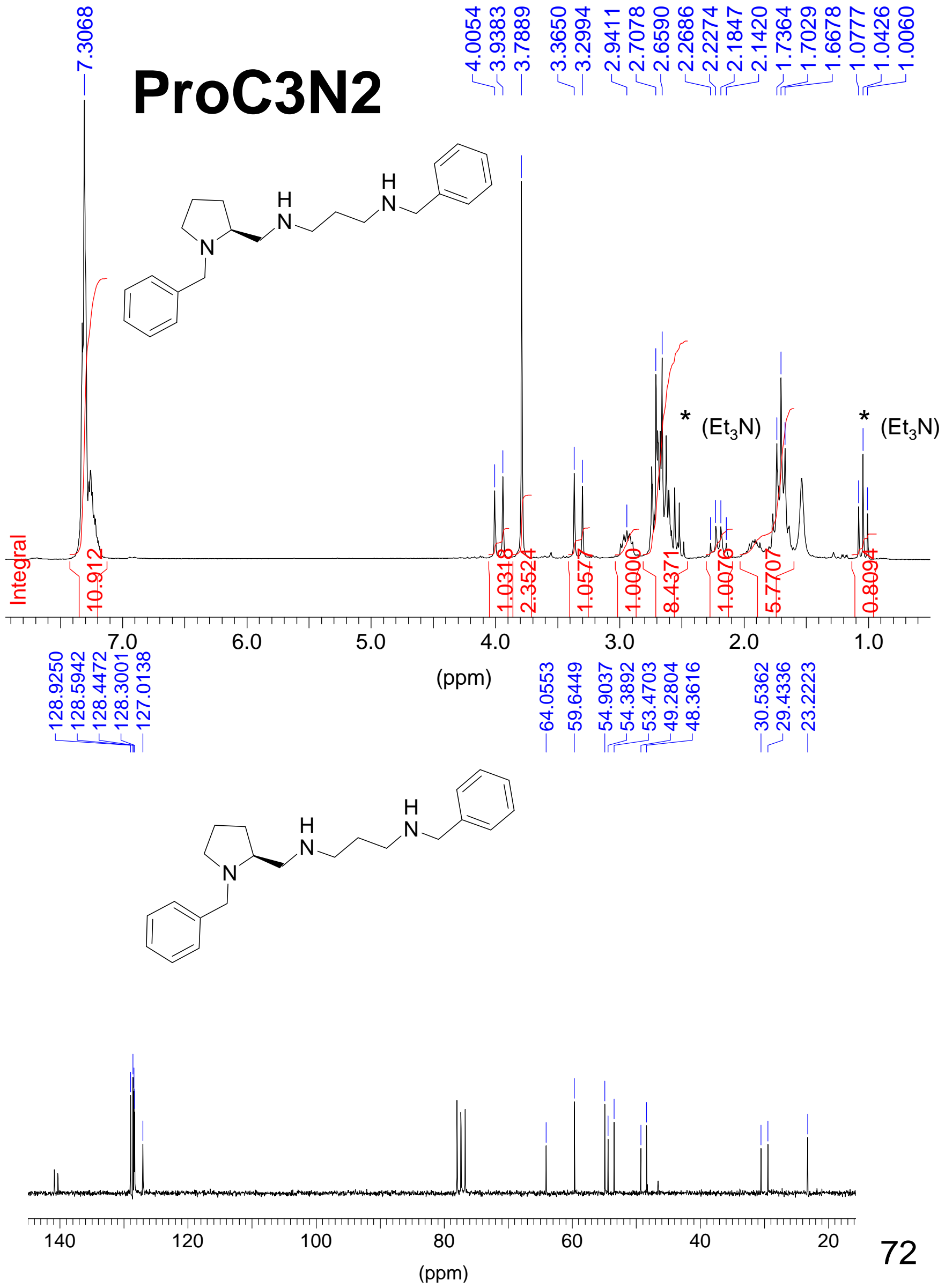




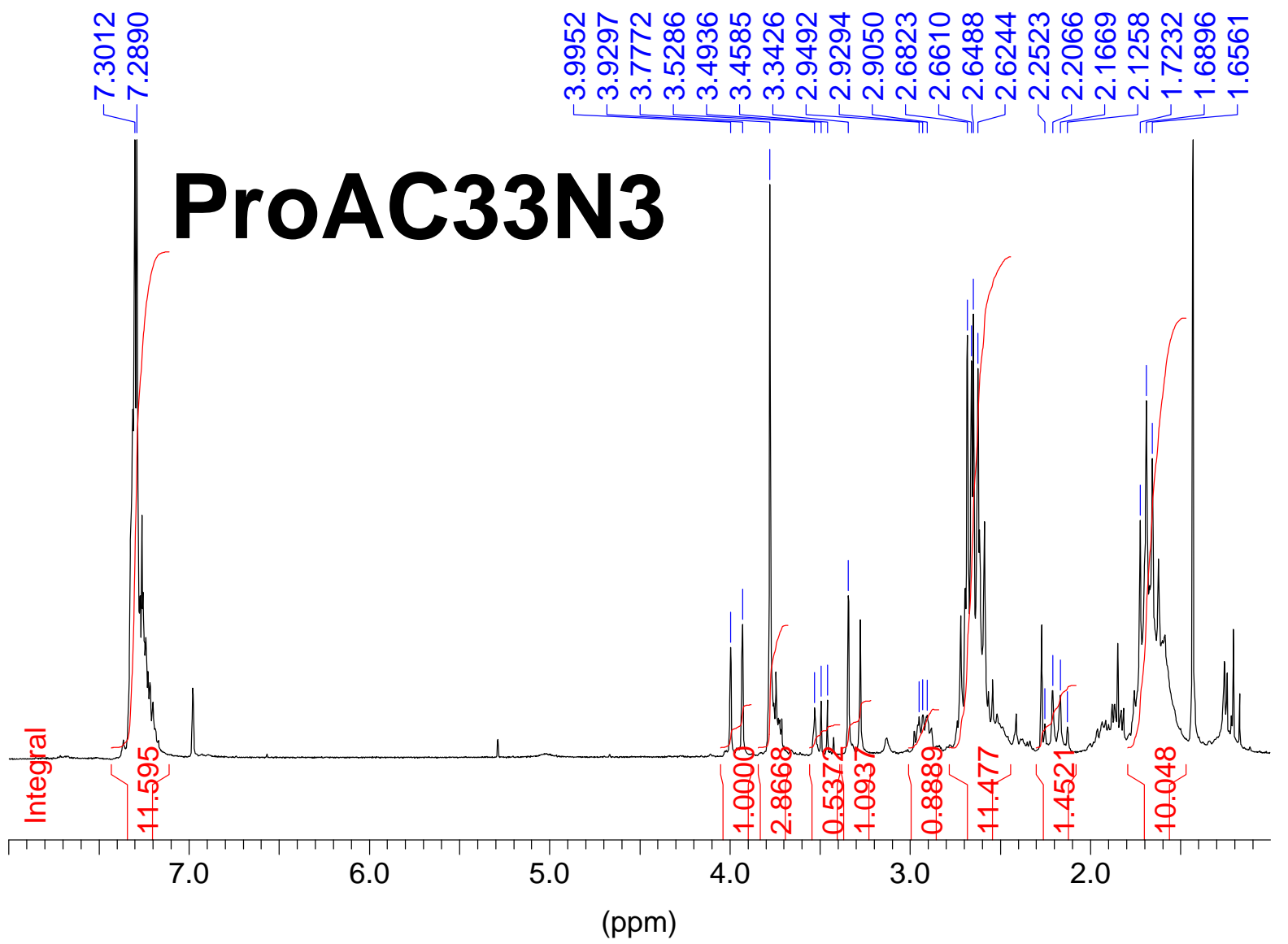

m노요요

L

กூ

จ

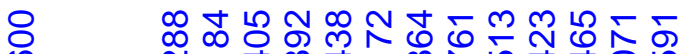

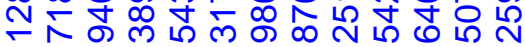

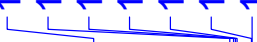

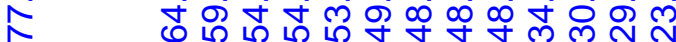
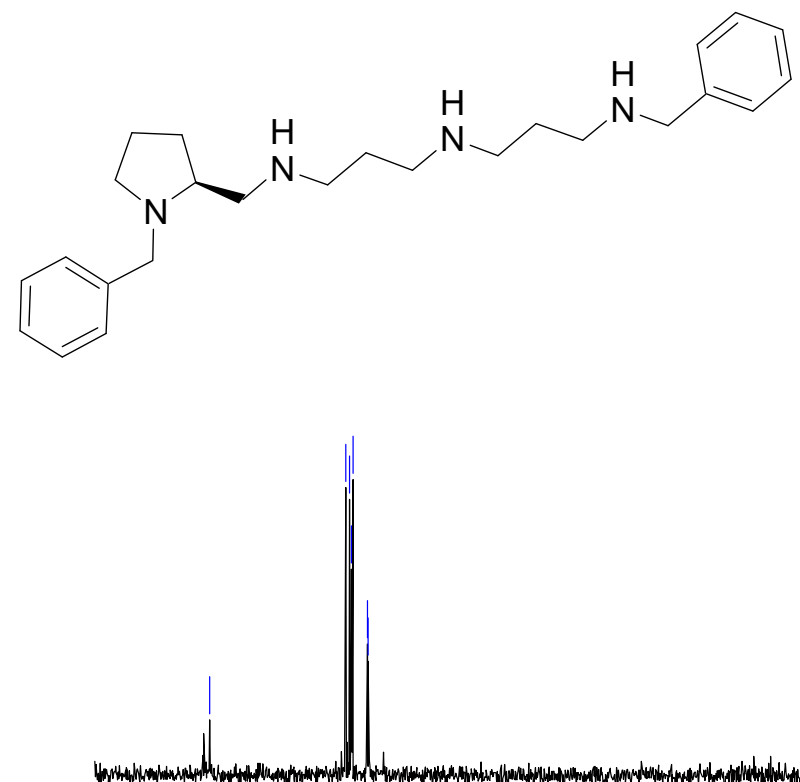

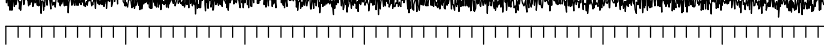


NMR assignments of molecules 1 were based on 1H (multiplicity of signals, integrals and coupling constants), COSY, HSQC and HMBC spectra. A representative NOESY spectrum is provided below.

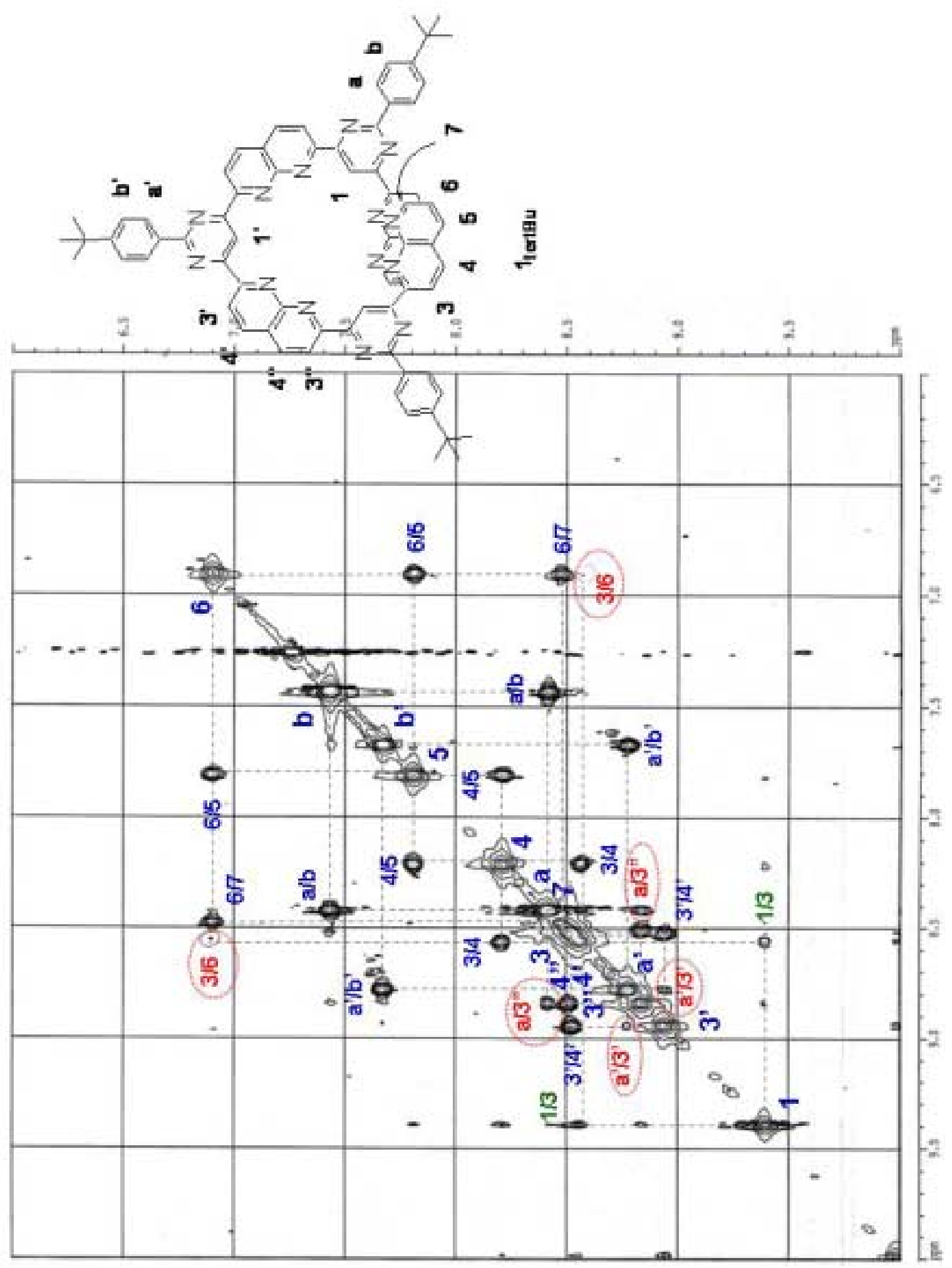

